\title{
Quantifying Hurricane Wind Speed with Undersea Sound
}

\author{
by \\ Joshua David Wilson \\ B.S., Mechanical Engineering, University of Idaho (1999) \\ Submitted in partial fulfillment of the requirements for the degree of \\ Doctor of Philosophy \\ at the \\ MASSACHUSETTS INSTITUTE OF TECHNOLOGY \\ and the \\ WOODS HOLE OCEANOGRAPHIC INSTITUTION
}

June 2006

(c) Joshua David Wilson, MMVI. All rights reserved.

The author hereby grants to MIT and WHOI permission to reproduce and distribute publicly paper and electronic copies of this thesis document in whole or in part.

Author

Joint Program in Oceanography/Applied Ocean Science and Engineering Massachusetts Institute of Technology and Woods Hole Oceanographic Institution June, 2006

Certified by

Nicholas C. Makris

Associngeressor of Mechanical Engineering Thesis Supervisor

Accepted by

Henrik Schmidt

unair, Jomt vommncee ror Appued Ocean Science and Engineering Massachusetts Institute of Technology and Woods Hole Oceanographic Institution

Accepted by

Chair, Committee on Graduate Students, Department of Mechanical Engineering 


\title{
Quantifying Hurricane Wind Speed with Undersea Sound
}

\author{
Joshua David Wilson \\ Submitted in partial fulfillment of the requirements \\ for the degree of Doctor of Philosophy \\ June, 2006
}

\begin{abstract}
Hurricanes, powerful storms with wind speeds that can exceed $80 \mathrm{~m} / \mathrm{s}$, are one of the most destructive natural disasters known to man. While current satellite technology has made it possible to effectively detect and track hurricanes, expensive 'hurricanehunting' aircraft are required to accurately classify their destructive power. Here we show that passive undersea acoustic techniques may provide a promising tool for accurately quantifying the destructive power of a hurricane and so may provide a safe and inexpensive alternative to aircraft-based techniques.

It is well known that the crashing of wind-driven waves generates underwater noise in the $10 \mathrm{~Hz}$ to $10 \mathrm{kHz}$ range. Theoretical and empirical evidence are combined to show that underwater acoustic sensing techniques may be valuable for measuring the wind speed and determining the destructive power of a hurricane. This is done by first developing a model for the acoustic intensity and mutual intensity in an ocean waveguide due to a hurricane and then determining the relationship between local wind speed and underwater acoustic intensity. Acoustic measurements of the underwater noise generated by hurricane Gert are correlated with meteorological data from reconnaissance aircraft and satellites to show that underwater noise intensity between 10 and $50 \mathrm{~Hz}$ is approximately proportional to the cube of the local wind speed. From this it is shown that it should be feasible to accurately measure the local wind speed and quantify the destructive power of a hurricane if its eye wall passes directly over a single underwater acoustic sensor. The potential advantages and disadvantages of the proposed acoustic method are weighed against those of currently employed techniques.

It has also long been known that hurricanes generate microseisms in the 0.1 to $0.6 \mathrm{~Hz}$ frequency range through the non-linear interaction of ocean surface waves. Here we model microseisms generated by the spatially inhomogeneous waves of a hurricane with the non-linear wave equation where a second-order acoustic field is created by first-order ocean surface wave motion. We account for the propagation of microseismic noise through range-dependent waveguide environments from the deep ocean to a receiver on land. We compare estimates based on the ocean surface wave field measured in hurricane Bonnie with seismic measurements from Florida.
\end{abstract}

Thesis Supervisor: Nicholas C. Makris

Title: Associate Professor of Mechanical Engineering 


\section{Acknowledgments}

First and foremost I thank my wife Becky for her unwavering love and support, my son Christian for his confidence that I am the smartest person in the world, and my daughter Annabelle for being the living example of determination.

I thank my thesis advisor, Prof. Nicholas C. Makris, for his guidance and insight. He was always helpful when I needed it and demanding when I deserved it.

I also thank the members of my committee; Prof. Kerry Emanuel of the Massachusetts Institute of Technology, Dr. Wade McGillis of the Woods Hole Oceanographic Institution and Prof. Purnima Ratilal of Northeastern University; for their interest in this project and for our many useful conversations.

Many individuals from other institutions contributed data, computational models, and advice to this project. They include Douglas H. Cato of Australia's Defense Science and Technology Organization, Christopher G. Fox and Tai-Kwan Andy Lau of the National Oceanic and Atmospheric Administration, and Il-Ju Moon of the University of Rhode Island.

The administrative staff at both the Massachusetts Institute of Technology and the Woods Hole Oceanographic Institution were extraordinary in their support. In particular I thank Carolyn Brooke, Geoff Fox, Marsha Gomes, Stephen Malley, Sabina Rataj, Leslie Regan, Ronni Schwartz and Julia Westwater.

My fellow students and friends provided much needed advice, support, critique, conversation and occasional distraction for which I am most grateful. For this I thank Ioannis Bertsatos, Tianrun Chen, Peter Daly, Joe Edwards, Srinivasan Jagannathan, Andrea Kraay, Yisan Lai, Sunwoong Lee, Monica Montenari, Luiz Sousa, Deanelle Symonds, Aaron Thode, Irena Veljkovic, Ding Wang, Jennifer Watson and Michele Zanoline.

Finally, I am grateful to have been awarded the Office of Naval Research Graduate Traineeship Award in Ocean Acoustics. I also thank the MIT Sea Grant office for 
funding portions of this research.

The wind bloweth where it listeth, and thou hearest the sound thereof, but canst not tell whence it cometh, and whither it goeth: so is every one that is born of the Spirit.

John $3: 8$ (KJV) 


\section{Contents}

1 Introduction $\quad 23$

1.1 Current Hurricane Classification Techniques . . . . . . . . . . . 24

1.2 Acoustic Field from Spatially Uncorrelated Surface Noise Sources _ . 26

1.3 Acoustic Field from the Interaction of Surface Gravity Waves on the Ocean Surface . . . . . . . . . . . . . . . . . . . 29

2 Ocean Acoustic Hurricane Wind Speed Quantification from Spatially Uncorrelated Surface Noise $\quad 31$

2.1 Introduction . . . . . . . . . . . . . . . . 31

2.2 Hurricane Structure and Current Classification Techniques . . . . 33

2.3 Wind Generated Surface Noise from Uncorrelated Surface Sources . . 36

2.4 Single Hydrophone Analysis $\ldots \ldots \ldots \ldots$

2.4 .1 Local Noise Dominates . . . . . . . . . . . . . . . 45

2.4.2 Separating the Effect of Attenuation by Bubbles from Local Waveguide Propagation . . . . . . . . . . . . 49

2.4.3 Accuracy of Underwater Acoustic Wind Speed Estimates . . . 54

2.4 .4 Practical Issues $\ldots \ldots \ldots \ldots 60 \ldots$

2.5 Hydrophone Array Analysis $\ldots \ldots \ldots$. . . . . . . . . 62

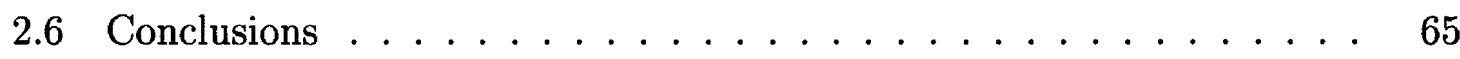


3 Quantifying Wind Speed in Hurricane Gert with Undersea Sound 67

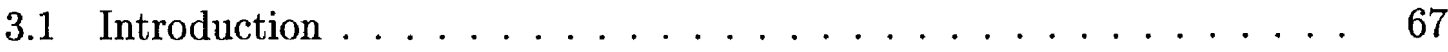

3.2 Methods . . . . . . . . . . . . . . . . 69

3.3 Results ........................ 73

3.4 Conclusions ....................... 80

4 Modeling Microseism Generation by Inhomogeneous Ocean Surface Waves in Hurricane Bonnie Using the Non-linear Wave Equation 83

4.1 Introduction . . . . . . . . . . . . . . . . 83

4.2 Ocean Surface Gravity Waves . . . . . . . . . . . . . . . . 86

4.3 Nonlinear Wave Equation . . . . . . . . . . . . . . 92

4.4 Power Spectral Density due to Ocean Surface Gravity Waves . . . . . 97

4.5 Microseismic Source Levels in Hurricane Bonnie . . . . . . . . . . . 106

4.6 Received Microseismic Levels in Florida Due to Hurricane Bonnie . . 108

4.7 Conclusions . . . . . . . . . . . . . . . . . 112

5 Conclusion $\quad 113$

A Noise Correlation from Random Surface Sources 115

B Asymptotic Variance for Wind Speed Estimates 119

C Review of "Microseism and infrasound generation by cyclones" by Bowen, Richard, Mancini, Fessatidis and Crooker

$\begin{array}{ll}\text { D Spectral Properties of Ocean Surface Waves } & 127\end{array}$

D.1 Homogeneous Surface Wave Fields . . . . . . . . . . . . 127

D.2 Inhomogeneous Surface Wave Fields . . . . . . . . . . . . 130

E Microseisms in a Range-independent half-space 135 
F Adiabatic Propagation of Generalized Rayleigh Waves in a Range Dependent Ocean Environment

G Review of "Emergence rate of the time-domain Green's function from the ambient noise cross-correlation function" by Sabra, Roux and Kuperman 


\section{List of Figures}

2-1 Hurricane wind speed as a function of distance from the hurricane center based on Holland's model[52] with parameters $A=72.44, B=$ $1.86, p_{c}=96300 \mathrm{~Pa}, p_{n}=100500 \mathrm{~Pa}$ and $\rho_{A I R}=1.15 \mathrm{~kg} / \mathrm{m}^{3}$. The zero wind speed region at the center of the hurricane $(0 \mathrm{~km})$ is called the eye and the high wind speed region $(10 \mathrm{~km})$ is the eye wall. The total destructive power of the hurricane is proportional to the cube of the maximum wind speed, which occurs in the eye wall.[53] $\ldots$. .

2-2 Cross section of the stratified ocean waveguide showing the geometry of the surface noise problem (Not to scale). On the surface is the area covered by the hurricane and surrounding area covered $5 \mathrm{~m} / \mathrm{s}$ ambient winds. The surface noise sources are modeled as a plane of monopoles a small depth $z_{0}$ below the surface and the sound field is measured by receivers and receiver arrays. . . . . . . . . . . .

2-3 Sound speed profiles $c(z)$ for the North Atlantic[82] and the Bay of Bengal[ $[69,114]$. The bottom has a density of $1.38 \mathrm{~g} / \mathrm{cm}$ and an attenuation of $0.3 \mathrm{~dB} / \lambda$ corresponding to the deep silty sediment layers of the Bay of Bengal[123, 48] and the North Atlantic Abyssal plain[49, 125]. The water has a density of $1 \mathrm{~g} / \mathrm{cm}$ and an attenuation of $6 \times 10^{-5} \mathrm{~dB} / \lambda .42$ 
2-4 Noise spectral level ( $\mathrm{dB}$ re $\left.I_{r e f}\right)$ in the North Atlantic (a) and (b) and the Bay of Bengal (c) and (d) for $n=3$. (a) and (c) show the level as a function of range at a depth of $200 \mathrm{~m}$ for 50, 400 and 3200 $\mathrm{Hz}$ frequencies. $L_{V}(\boldsymbol{\rho})=10 \log \left(V(\boldsymbol{\rho}) / V_{\text {ref }}\right)$ is plotted for comparison where $V_{\text {ref }}=5 \mathrm{~m} / \mathrm{s} . L_{V}=0$ is equivalent to $V=5 \mathrm{~m} / \mathrm{s}$ and $L_{V}=10$ is equivalent to $V=50 \mathrm{~m} / \mathrm{s}$. (b) and (d) show the level as a function of range and depth at $50 \mathrm{~Hz}$. In both waveguide environments the noise level closely follows the local wind speed. In the North Atlantic there is a convergence zone structure corresponding to sound that propagates from the hurricane's eye wall via refraction. Note the convergence zone near the surface at a range of $257 \mathrm{~km}$ and the ray vertex depth of 4.7

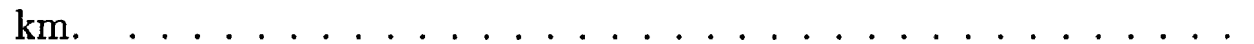


2-5 Error induced by the local area approximation (Eq. (2.9)) as a function of local source area radius $R_{\text {local }}$ for a single sensor under the maximum winds in the eye wall of a hurricane. Curves are shown for the North Atlantic and the Bay of Bengal environments used in this paper as well as for infinite half-space and shallow water continental shelf environments. Plots are given for sensor depths of $100 \mathrm{~m} \mathrm{((a)}$ and (c)) and $800 \mathrm{~m} \mathrm{((b)}$ and (d)) and for frequencies of $50 \mathrm{~Hz}((\mathrm{a})$ and (b)) and 400 $\mathrm{Hz}((\mathrm{c})$ and $(\mathrm{d}))$. While these plots are given for $n=3$ the difference for values $n=1$ to 4 is less than $0.1 \mathrm{~dB}$. The North Atlantic, Bay of Bengal and infinite half-space environments are very similar. In these deep-water environments, for the shallow $100 \mathrm{~m}$ sensor depth, we see that for $R_{\text {local }}$ over roughly $300 \mathrm{~m}$ the error from this approximation is negligible. For the deeper $800 \mathrm{~m}$ sensor depth, the $R_{\text {local }}$ for which the error is negligible is roughly $2 \mathrm{~km}$. In shallow water the error in the local area approximation is higher leading to a larger $R_{\text {local }}$. This is likely due to the strong reflection of sound off bottom. In deep water environments bottom reflections have little effect and most of the sound measured by a receiver propagates via direct path from the surface source. . . . . . . . . . . . . . . . . . . . . 
2-6 Simulated noise spectral level ( $\mathrm{dB}$ re $\left.I_{r e f}\right)$ in the North Atlantic for range-independent winds as a function of wind speed and frequency including attenuation by sea-surface bubbles assuming $n=3$ from Eq. (2.5). Below $100 \mathrm{~Hz}$ the power-law relationship between noise intensity and wind speed is unaffected by bubble attenuation even up to the 80 $\mathrm{m} / \mathrm{s}$ wind speeds of a hurricane. As frequency increases, attenuation affects the noise level at progressively lower wind speeds. For a given frequency the noise level increases linearly with wind speed, peaks, and then decays exponentially. . . . . . . . . . . . . . .

2-7 (a) Noise spectral level ( $\mathrm{dB}$ re $\left.I_{r e f}\right)$ as a function of wind speed at several frequencies, assuming $n=3$. The black curves show the attenuation, caused by bubbles, at $50 \mathrm{~Hz}, 400 \mathrm{~Hz}$ and $4 \mathrm{kHz}$. The range of wind speeds typical of a hurricane is also shown. (b) Noise spectral level curves as a function of frequency for typical hurricane wind speeds of 30,50 and $80 \mathrm{~m} / \mathrm{s}$. The black curves show the full areal integration from Eq. (2.5) and the grey curves show the first-order approximation of the field given by Eq. (2.19) with Eqs. (2.15), (2.20) and (2.21). .

2-8 The percent RMSE $\nu_{r m s e}$ (a) and percent bias $\nu_{\text {bias }}$ (b) of the wind speed estimate $\hat{V}$ where attenuation by sea-surface bubbles is insignificant, evaluated numerically from Eqs. (2.5) and (2.27). For timebandwidth products $\mu>5$ the estimate becomes unbiased and the RMSE attains the Cramer-Rao Lower Bound. Piggott[102] and Perrone[96] have measured wind noise level with standard deviations less than 1 $\mathrm{dB}$ which corresponds to $\mu>19$. For $\mu=19$ the percent RMSE in the wind speed estimate ranges from 6 to $25 \%$ depending on $n$ which is a significant improvement over the primary satellite classification method. . . . . . . . . . . . . . . . . . 
2-9 The percent RMSE $\nu_{r m s e}$ (a) and percent bias $\nu_{b i a s}$ (b) of the wind speed estimate $\hat{V}$ including the effect of attenuation calculated numerically from Eqs. (2.5) and (2.27), assuming $n=3$, at $f=400 \mathrm{~Hz}$ where $V_{\max }=58 \mathrm{~m} / \mathrm{s}$. The error and bias increase for $V \approx V_{\max }$ but for $\mu>5$ and for values of $V$ where $\nu_{b i a s}<1 \%$ the percent RMSE decreases and attains the Cramer-Rao Lower Bound. For spectral intensity measurements with $\mu=19$ the percent RMSE in this example is between 9 and $20 \% \ldots \ldots \ldots \ldots \ldots$

2-10 Angular spectral density level $10 \log (B(\Omega, f))\left(\mathrm{dB}\right.$ re $\left.\frac{\rho_{\mathrm{w}} \mathrm{C} \mathrm{I}_{\mathrm{ref}}}{\mathrm{s}^{2}}\right)$ at $100 \mathrm{~Hz}$ for a 64 -element $\lambda / 2$-spaced horizontal broadside array as a function of steering angle for hurricane generated noise in the North Atlantic at ranges of $257 \mathrm{~km} \mathrm{(a),} 289 \mathrm{~km} \mathrm{(b),} \mathrm{and} 385 \mathrm{~km} \mathrm{(c)} \mathrm{from} \mathrm{the} \mathrm{eye} \mathrm{of}$ the hurricane, assuming $n=3$. Ranges of 257 and $385 \mathrm{~km}$ correspond to the fourth and sixth convergence zones from the center of the hurricane. $289 \mathrm{~km}$ is exactly between the fourth and fifth convergence zones. Curves are shown for a powerful $72 \mathrm{~m} / \mathrm{s}$ hurricane, a medium $50 \mathrm{~m} / \mathrm{s}$ hurricane and a weak $33 \mathrm{~m} / \mathrm{s}$ hurricane. The angular spectral density level from ambient noise is plotted for comparison. A steering angle of $0^{\circ}$ corresponds to the array steered toward the calm eye of the hurricane and the powerful eye wall is located at $\pm 3^{\circ}$. This array has an angular resolution of $1.8^{\circ}$ which at a range of $257 \mathrm{~km}$ corresponds to a $8 \mathrm{~km}$ spatial resolution. . . . . . . . . . . . . . 
3-1 Spectrogram of the hurricane noise in $\mathrm{dB}$ re $\mu \mathrm{Pa}^{2} / \mathrm{Hz}$, from 1 to 50 $\mathrm{Hz}$, received by the NOAA hydrophone on 15 Sept. Above $10 \mathrm{~Hz}$ the maximum between 13:00 and 16:00 correspond to the powerful winds of the hurricane's eye-wall. At $1 \mathrm{~Hz}$ we see noise most likely due to cable strum. There seems to be some hurricane related noise between 1 and $10 \mathrm{~Hz}$, however it does not correlate well with wind speed and may be caused by nonlinear surface wave interaction as discussed in the next chapter. . . . . . . . . . . . . . . . . . . .

3-2 Log of the noise spectral intensity $I$ (solid blue) in Watts $/ \mathrm{m}^{2} \mathrm{~Hz}$, from 10 to $50 \mathrm{~Hz}$, received by the NOAA hydrophone on 15 Sept. The expected intensity (red dashed line) is based on the hindcast wind speed $V$ in $\mathrm{m} / \mathrm{s}$ above the hydrophone given the best fit relation $\log \left[I /\left(1 \mathrm{Watt} / \mathrm{m}^{2} \mathrm{~Hz}\right)\right]=$ $n \log [V /(1 \mathrm{~m} / \mathrm{s})]+b$ where $n=3.36$ and $b=-14.46$. The maxima at 13:30 and 15:30 correspond to the powerful winds of the hurricane's eye-wall and the minimum at 14:30 corresponds to the hurricane's eye. The percent root-mean-square error between the two curves is $5 \%$. .

3-3 Wind speed in $\mathrm{m} / \mathrm{s}$ in hurricane Gert as a function of position relative to the center or 'eye'. As hurricane Gert passed over the acoustic sensor the sensor effectively sliced a path through the hurricane. The lines show the paths estimated based on Satellite (dash-dot), Aircraft (dashed) and Acoustic best fit (solid) respectively. . . . . . . . . .

3-4 The $\log$ of the noise spectral intensity $I$ in Watts $/ \mathrm{m}^{2} \mathrm{~Hz}$ versus the $\log$ of the surface wind speed $V$ in $\mathrm{m} / \mathrm{s}$ at the NOAA hydrophone (circles) based on the best fit hurricane track. The best fit linear regression shows a $\log \left[I /\left(1 \mathrm{Watt} / \mathrm{m}^{2} \mathrm{~Hz}\right)\right]=3.36 \log [V /(1 \mathrm{~m} / \mathrm{s})]-14.46$ relationship between intensity and wind speed with a percent root-mean-square error of only $5 \% \ldots \ldots \ldots \ldots \ldots \ldots \ldots$ 
3-5 The upper plot gives the root-mean-square error (RMSE) of the linear regression for each possible hurricane track. The y-axis is the north-south distance between the hydrophone and the possible hurricane track and the $\mathrm{x}$-axis is the speed at which the hurricane was moving. The minimum RMSE of $0.76 \mathrm{~dB}$, indicated y the + symbol, is taken to represent the 'best fit' hurricane track. Note that the RMSE is small, less than $0.8 \mathrm{~dB}$, over a range of possible tracks. The lower plot gives the slope $n$ of the linear regression for each possible hurricane track with the same $\mathrm{x}$ and $\mathrm{y}$ axes as the upper plot. For the best track the slope $n=3.36$. For the range of tracks with a RMSE less than $0.8 \mathrm{~dB}$, the slope of the regression could vary from 3 to 3.6 . . .

3-6 Log of noise intensity $I$ versus $\log$ of hindcast local wind speed $V$ based on the best-fit track (red circles). Also plotted is the log of noise intensity $I$ versus $\log$ of local wind speed $V$ measured by Cato and Tavener [23] (blue circles) at lower non-hurricane wind speeds off Australia. The linear regression shows a $\log \left(I / 1 \mathrm{Watt} / \mathrm{m}^{2} \mathrm{~Hz}\right)=$ $3.34 \log (V / 1 \mathrm{~m} / \mathrm{s})-14.5$ relationship between noise intensity $I$ and wind speed $V . \quad \ldots \ldots \ldots \ldots \ldots \ldots \ldots \ldots \ldots$ 
4-1 (A) Wind speed in $\mathrm{m} / \mathrm{s}$ and (B) microseismic source level $L_{B_{m m}}\left(\omega, z_{s}=\right.$ 0 ) at $\omega=1.26 \mathrm{rad} / \mathrm{s}(f=0.2 \mathrm{~Hz})$ in $\mathrm{dB}$ re $P a^{2} s / m^{4}$ from Eq. (4.39) at 1200 on 24 August 1998 as a function of latitude and longitude. The arrow indicates the direction hurricane Bonnie was moving. The letters (a), (b), (c) and (d) represent features of interest; (a) indicates the location of maximum wind speed, (b) indicates the eye where wind speed is zero, and (c) and (d) indicate peaks in the microseismic source level. This figure shows that, while a hurricane can produce significant microseismic source levels (B), these level do not directly follow the wind speeds $(\mathrm{A})$ in the hurricane. $\ldots \ldots \ldots \ldots \ldots$

4-2 The wave-height power spectral level $\left(10 \log S_{m m}(\boldsymbol{\kappa}) /\left(\mathrm{m}^{2} /\left(\mathrm{rad}^{2} / \mathrm{m}^{2}\right)\right)\right)$ in $\mathrm{dB} \mathrm{re} \mathrm{m}^{2} /\left(\mathrm{rad}^{2} / \mathrm{m}^{2}\right)$ at the locations of interest (a), (b), (c), and (d) given in Fig. 4.2 at 1200 on 24 August 1998. The peak in the spectra is at roughly $\kappa=0.04 \mathrm{rad} / \mathrm{m}$ or $\sigma=0.63 \mathrm{rad} / \mathrm{s}$ which corresponds to an acoustic frequency of $\omega=1.26 \mathrm{rad} / \mathrm{s}$ or $f=0.2 \mathrm{~Hz}$. At some locations (b, c, and d) there are waves propagating with opposing wavenumber vectors $\kappa$, while at other locations (a), most of the waves propagate in the same direction. From Eq. (4.37) we expect these locations with opposing waves to produce the greatest microseismic source levels and shown in Fig. 4.2(B). . . . . . . . . . . . . . . . . . . 
4-3 Geometry of the hurricane wave field and ocean waveguide (not to scale). In our model the waveguide environment may be range dependent and in this paper we consider the example of an upslope propagation from the deep North Atlantic to Florida. The range and ocean depth parameters $\mathrm{R}$ and $\mathrm{d}$ are given in Table 4.6. The depth of the receiver below the ocean bottom of $162 \mathrm{~m}$ corresponds to the depth below the earth surface of the actual seismometer in Florida. The compression wave speeds $c_{p}$ are 1500 and $5200 \mathrm{~m} / \mathrm{s}$ in the water and bottom respectively. The shear wave speed $c_{s}$ in the bottom is 3000 $\mathrm{m} / \mathrm{s}$. The densities $\rho$ in the water and bottom are 1.0 and $2.5 \mathrm{~g} / \mathrm{cm}$ respectively. $\ldots \ldots \ldots \ldots \ldots \ldots \ldots \ldots$

4-4 The ocean depth (solid line) between hurricane Bonnie and the seismometer in Florida at noon on 24 August. Also shown is the ocean depth for the idealized up-sloping environment used to calculate the Green functions in Section 4.6 (dashed line). The scale of the figure makes the actual slope appear to change rapidly, however, the maximum slope of the ocean floor is roughly $3^{\circ} \ldots \ldots \ldots \ldots$ 
4-5 Horizontal (a-d) and vertical (e-h) velocity spectra, as a function of seismic frequency $(f=2 \pi \omega)$, modeled (red) and measured (blue) at the seismometer in Florida at four times on 24 August 1998. The dashed blue lines represent portions of the data that where corrupted by nonhurricane related ambient noise. Note that the peak in the spectra is at roughly $f=0.2 \mathrm{~Hz}$. This frequency corresponds to the peak in the wave-height power spectral level at $\kappa=0.04 \mathrm{rad} / \mathrm{m}$ (surface wave frequency $f=2 \pi \sigma=0.1 \mathrm{~Hz}$ ) seen in Fig. 4.2 and in plots (i-1). The surface wave-height power spectral level $\left(10 \log S_{m m}(\kappa) /\left(m^{2} /\left(\operatorname{rad}^{2} / m^{2}\right)\right)\right)$ in plots (i-1) is taken at the center of the storm and averaged over wave propagation direction. . . . . . . . . . . . . . .

G-1 The RHS and LHS in log (upper plot) and linear (lower plot) domains from 13.33 to 13.4 seconds. We see that the peaks in the curves occur at the same times but that the amplitudes of the peaks differ significantly. We also see that the RHS peaks are higher for the earlier low-order modes while the LHS peaks are higher for the later high-order modes. Note that the RHS in the lower plot has been scaled by a factor of five so that it can be compared against the LHS. . . . . . . . . . . . .

G-2 The RHS and LHS (upper plot) over a longer time duration. We continue to see the general trend where the peaks of the RHS are higher for low-order early arrivals and lower for later high-order arrivals. This can also be seen in the lower plot which shows the difference between the RHS and LHS. . . . . . . . . . . . . . . . . . . . . . . . 153

G-3 The weighting term $\frac{\left|u_{m}\left(z_{s}\right)\right|^{2}}{\alpha_{m} \kappa_{m}}$ as a function of mode number $m$ at several frequencies in an isovelocity waveguide. The source depth $z_{s}<<\lambda$ typical of ocean surface noise. . . . . . . . . . . . . . . . 


\section{List of Tables}

4.1 Previous microseism models and their assumptions $\ldots \ldots \ldots$

4.2 Parameters for Hurricane Bonnie on 24 August 1998 . . . . . . 109 


\section{Chapter 1}

\section{Introduction}

In this thesis we theoretically and experimentally study the noise generated by a hurricane and show that passive undersea acoustic techniques may provide a promising tool for accurately quantifying the destructive power of a hurricane and may provide a safe and inexpensive alternative to aircraft-based techniques. While current satellite technology has made it possible to effectively detect and track hurricanes, expensive 'hurricane-hunting' aircraft are required to accurately classify its destructive power.

Quantification of a hurricane's total destructive power, which is proportional to the cube of the hurricane's maximum wind speed [52], is critical for hurricane planning. The destructive power of a tropical cyclone was recently demonstrated by hurricane Katrina which caused over 1000 fatalities[29] and an estimated economic loss of 100 billion dollars[10] and in 1970 a hurricane killed over 300,000 people in Bangladesh in[33]. Prior to Katrina the United States Commission on Ocean Policy emphasized the need for more accurate quantifications of hurricane destructive power to improve disaster planning[133]. Inaccurate quantifications can lead to poor forecasting and unnecessary evacuations, which are costly, or missed evacuations, which can result in loss of life[36]. These fatalities and costs can be reduced if the public is given timely and accurate advanced warning, but this depends on the ability to accurately quantify 
hurricane wind speed while it is still far from land. Current classification and warning systems save an average $\$ 2.5$ billion each year in the United States alone[133] and more accurate systems could save even more.

\subsection{Current Hurricane Classification Techniques}

Currently there are two primary tools used by meteorologists for detecting and classifying hurricanes in the ocean; satellites and aircraft. Satellites are useful for detecting and locating hurricanes but their poor spatial resolution in measuring wind speeds limits their ability to accurately classify the hurricane. Aircraft are much better for classifying hurricanes, however, their expense means that their use is not feasible for most countries[33]. Also their limited endurance means that they cannot monitor the hurricane continuously.

The Dvorak method $[31,32,131]$ is the most common method for classifying a hurricane's destructive power. This method, where hurricane cloud features observed in satellite images are interpreted to estimate wind speed and classify destructive power, can yield errors in wind speed estimates exceeding $40 \%$ [93, 43, 12, 4, 121] when compared to the best-estimate wind speed from aircraft measurements. For example, of the eight North Atlantic hurricanes of 2000, three of them [93, 43, 12] experienced Dvorak errors over $40 \%$ and three more $[94,72,120]$ experienced Dvorak errors over $20 \%$. Despite these errors, the Dvorak method is still the primary technique for classifying the destructive power of a hurricane from satellite measurements [44]. A satellite-based pattern-recognition technique similar to the Dvorak method using SSM/I satellite microwave (85 GHz) instead of optical and infrared images has recently been developed but gives similar errors as the Dvorak method [7].

Satellite classification of hurricanes with Microwave Sounding Units (MSU) [130] is secondary to the primary Dvorak method [44] due to the limited spatial resolution 
of the unit. The $55 \mathrm{GHz}$ microwave radiation given off by warm air in the hurricane's eye is used to estimate temperature and then infer the hurricane's power. Because of the small size of the satellite array unit the spatial resolution of the measurement is about $48 \mathrm{~km}$ [65], which is often larger than the diameter of the eye, resulting in a blurred image of the hurricane and potentially leading to errors in the estimate of the destructive power $[130,65]$.

Other satellite techniques for estimating hurricane wind speed and destructive power are currently being studied. For an overview see the article by Katsaros, Vachon, Liu and Black [59]. These techniques, however, are still under development and are not yet being used operationally for hurricane classification and disaster planning [41].

More accurate classification can be achieved by flying specialized 'hurricane-hunting' aircraft, like the Air Force's WC-130 and NOAA's WP-3, through the high winds of a hurricane [41]. Using on-board sensors and expendable dropsondes, accurate wind speed estimates with errors less than $5 \mathrm{~m} / \mathrm{s}$ can be obtained [41]. Unfortunately the expense of these aircraft prohibits their routine use outside of the United States [33]. For example, the cost to purchase a WC-130 aircraft is roughly $\$ 82$ million [2] (adjusted for inflation to year 2005 dollars) and the deployment cost is $\$ 155,000$ per flight [9]. Given these limitations in current capability, the United States Commission on Ocean Policy has recommended that future ocean observing systems be used to improve weather related warnings [133]. We experimentally and theoretically demonstrate that underwater acoustic measurements of noise intensity may provide a useful technique for hurricane wind speed estimation. 


\subsection{Acoustic Field from Spatially Uncorrelated Sur- face Noise Sources}

Sea-surface agitation from the action of wind and waves is a dominant source of ambient noise in the ocean $[67,136]$ in the $10 \mathrm{~Hz}$ to $10 \mathrm{kHz}$ frequency range. We show that it may be practical to inexpensively determine local wind speed and quantify the destructive power of a hurricane by measuring this noise which can be described as a sum of fields radiated from many random sources on the sea surface $[26,76,70,19$, $95,81]$. If the surface noise sources have the same statistical distribution, Ingenito and Wolf have shown that the wind-generated noise spectral intensity is the product of two separate factors, a waveguide propagation factor and a "universal ambient noise" [57] source factor which is a function of wind speed but otherwise is expected to be effectively independent of horizontal position.

Shaw, Watts and Rossby[118] first considered the concept of using underwater sound to estimate wind speed for spatially uniform wind speed distributions. They found sound pressure level in $\mathrm{dB}$ to be linearly related to the $\log$ of the wind speed and the idea of a universal ambient noise source factor was implicit in their approach. We will show that the slope of their linear relationship corresponds to the universal ambient noise factor and the intercept to the waveguide propagation factor. Evans, Watts, Halpern and Bourassa demonstrate that these wind speed estimates could be made to within $\pm 1 \mathrm{~m} / \mathrm{s}$ at low 5 to $10 \mathrm{~m} / \mathrm{s}$ wind speeds [38], which is much less than is experienced in a hurricane.

Many experiments have been conducted to determine the relationship between local wind speed and underwater noise intensity as noted in Ref. [18]. A common difficulty in these experiments has been contamination from shipping noise $[18,61]$. This typically leads to poorer correlation and greater variance in estimates of the relationship between wind speed and noise intensity [18]. Two experimental stud- 
ies conducted over many months that minimized this contamination show that a consistent high-correlation power-law relationship exists [102, 38]. They also show underwater noise intensity to be linearly proportional to wind speed to a frequency dependent power, ranging from two to four, for wind speeds between 5 and $20 \mathrm{~m} / \mathrm{s}$. It is possible that at higher frequencies attenuation by bubbles could cause a roll-off in the power-law relationship [139]. This attenuation, however, is insignificant at low frequencies and can be accurately measured and modeled at high frequencies.

In this thesis we present the first published data relating ambient noise and wind speed in a hurricane. In 1999 an autonomous underwater acoustic sensor package in the North Atlantic recorded the underwater noise from crashing wind-driven waves as hurricane Gert passed overhead. By correlating this noise with meteorological data from reconnaissance aircraft and satellites we show that underwater noise intensity between 10 and $50 \mathrm{~Hz}$ is approximately proportional to the cube of the local wind speed. Passive underwater acoustic intensity measurements from a single sensor may then be used to estimate hurricane wind speed to within a $5 \%$ error and from this accurately quantify the destructive power of the hurricane.

Based on theoretical and empirical evidence we find that it may be possible to estimate local hurricane wind speed by generalizing the approach of Shaw, Watts and Rossby [118]. We show that the wind-generated noise received by a single underwater acoustic sensor in a hurricane can be well approximated by sea-surface contributions so local that wind speed and surface source intensity can be taken as nearly constant. With these findings, noise intensity can be well approximated as the product of a local universal ambient noise source factor and a waveguide propagation factor even for the range-dependent wind speeds of a hurricane.

At low frequencies, below roughly $100 \mathrm{~Hz}$, we show that attenuation by windinduced bubbles in the upper-ocean boundary layer should be insignificant even in hurricane conditions. Temporal variations in underwater noise intensity should then 
be primarily caused by the universal ambient noise source factor which is expected to depend on local wind speed and will vary as a hurricane advects over a fixed receiver. By extrapolating known relationships [102] between wind speed and noise level in this frequency range, the ambient noise level should increase monotonically with wind speed, and it should be possible to directly estimate local wind speed from measured noise level.

At higher frequencies temporal variations in underwater noise intensity may also be caused by attenuation due to scattering from bubbles in the upper-ocean boundary layer. This attenuation increases with wind speed and acoustic frequency. Farmer and Lemon [40] experimentally show that this leads to a frequency dependent peak in noise level versus wind speed at frequencies above $8 \mathrm{kHz}$ and wind speeds above $15 \mathrm{~m} / \mathrm{s}$. We analytically show that such a peak may also exist for frequencies above $100 \mathrm{~Hz}$ in typical hurricane wind speeds. Since the shape of the ambient noise versus wind speed curve and the location of its peak vary strongly with frequency, we show that wind speed may still be unambiguously estimated from broadband ambient noise measurements in hurricane conditions above $100 \mathrm{~Hz}$ once the corresponding universal source dependence is empirically determined.

The accuracy of underwater acoustic wind speed estimates depends on the signal to noise ratio (SNR) of the underwater ambient noise intensity measurements upon which they are based. Piggott [102] and Perrone [97] have consistently measured wind noise with a standard deviation of less than one $\mathrm{dB}$ and in measurements of hurricane Gert we find standard deviations of $0.7 \mathrm{~dB}$, as expected from theory where the variance of the intensity measurement can be reduced by stationary averaging $[99,80,81]$. For previously measured power-law relationships that range from quartic to square $[102,38]$, a one $\mathrm{dB}$ standard deviation in sound pressure level corresponds to a $6 \%$ to $12 \%$ respective error in estimated wind speed. The 3.3 power law and $0.7 \mathrm{~dB}$ standard deviation measured in hurricane Gert results in only a $5 \%$ error in 
estimated wind speed.

Ocean acoustics then has serious potential for providing accurate and inexpensive hurricane classification estimates. Since a single hydrophone effectively measures only the local surface noise, it will effectively cut a swath through the hurricane yielding local wind speed estimates as the storm passes over. At low frequencies, current evidence suggests a simple power-law relationship between noise intensity and wind speed. At higher frequencies, a frequency-dependent roll-off is expected in the relationship due to attenuation by bubbles. Wind speed can still be uniquely estimated, however, by making broad-band measurements at higher frequency.

In Chapter 2 we review models for range-dependent noise in the ocean and develop a model for wind generated noise from a hurricane for both single sensors and arrays. We use this model to demonstrate the potential usefulness of quantifying hurricane wind speed with underwater acoustic sensors. We also review past experiments where the relationship between underwater noise intensity and wind speed was measured.

In Chapter 3 we present data from an autonomous underwater acoustic sensor package in the North Atlantic that recorded the underwater noise from crashing winddriven waves as hurricane Gert passed overhead. This data further demonstrates the potential usefulness of classifying hurricanes with underwater acoustic sensors.

\subsection{Acoustic Field from the Interaction of Surface Gravity Waves on the Ocean Surface}

Hurricanes also generate seismic noise, commonly referred to as microseisms, in the 0.1 to $0.6 \mathrm{~Hz}$ frequency range. In Chapter 4 we describe the microseisms generated by the spatially inhomogeneous waves in a hurricane. Using the ocean surface directional wave spectrum in hurricane Bonnie $[142,88]$ we hindcast the microseismic field and compare it with seismic measurements from Florida. Previously hurricane 
surface directional wave spectra had not been adequately measured or modeled so that researchers had to rely on assumed spectra in order to model hurricane microseisms [78]. This analysis is useful because microseisms are a primary cause of noise in seismic measurements $[74,107,105]$ that raise the detection threshold for monitoring earthquakes [135] and tsunamis. Historically microseismic measurements have also been used to track hurricanes $[107,45]$ although that task is now accomplished using satellites. We find that, due to the nonlinear nature of microseism generation, measurements of the microseismic field will probably not be useful for hurricane wind speed estimation.

We present an analytic expression for microseism generation by the spatially inhomogeneous waves typical in a hurricane based on the non-linear wave equation where a second-order seismo-acoustic field is generated by a source distribution which depends on the first-order ocean surface wave motion. The seismo-acoustic field at a receiver can then be expressed as the integral over the source distribution multiplied by the waveguide Green function. This expression is ideal for hurricane generated microseisms since it can be used to calculate the acoustic field due to spatially inhomogeneous surface waves. Also, this expression may be used in range-dependent waveguide environments as is the case when a hurricane at sea generates microseisms that propagate up the continental margin to a receiver on land.

Based on the wave-height spectra in hurricane Bonnie, we calculate the microseismic source levels generated by the nonlinear interaction of the ocean surface waves. Our derivation shows that microseisms are generated by the non-linear interaction of ocean surface waves with roughly the same wavelength but nearly opposing propagation directions. This is in agreement with earlier works $[86,78]$. Recent measurements $[142,132]$ and models [88] of surface directional wave height spectra in hurricane Bonnie show complex patterns with the opposing surface waves necessary to generate microseisms. 


\section{Chapter 2}

\section{Ocean Acoustic Hurricane Wind Speed Quantification from Spatially Uncorrelated Surface Noise}

\subsection{Introduction}

A case is made that it may be practical to inexpensively determine local wind speed and quantify the destructive power of a hurricane by measuring its underwater acoustic noise intensity. The dominant source of ambient noise in the ocean is sea-surface agitation from the action of wind and waves [67, 136]. This noise can be described as a sum of fields radiated from many random sources on the sea surface $[26,76,70,19,95,81]$. If the surface noise sources have the same statistical distribution, Ingenito and Wolf have shown that wind-generated noise spectral intensity is the product of two separate factors, a waveguide propagation factor and a "universal ambient noise" [57] source factor which is a function of wind speed but otherwise is 
expected to be effectively independent of horizontal position.

Shaw, Watts and Rossby [118] found sound pressure level in $\mathrm{dB}$ to be linearly related to the $\log$ of the wind speed and from this developed the concept of using underwater sound to estimate wind speed for spatially uniform wind speed distributions. We show that the slope of their linear relationship corresponds to the universal ambient noise factor described by Ingenito and Wolf [57] and the intercept to the waveguide propagation factor. As discussed in Chapter 1, it was later demonstrated that these estimates could be made to within $\pm 1 \mathrm{~m} / \mathrm{s}$ in the 5 to $10 \mathrm{~m} / \mathrm{s}$ wind speed range [38].

By generalizing the approach of Shaw, Watts and Rossby [118] we find that it may be possible to estimate local hurricane wind speed. The wind-generated noise received by a single underwater acoustic sensor in a hurricane can be well approximated by sea-surface contributions so local that wind speed and surface source intensity can be taken as nearly constant. Noise intensity can then be well approximated as the product of a local universal ambient noise source factor and a waveguide propagation factor even for the range-dependent wind speeds of a hurricane.

In this chapter we review models for the spatial wind speed dependence of a hurricane that will be used to model ambient noise. We also review past experiments that measured the relationship between underwater noise intensity and wind speed. We review models for range-dependent noise in the ocean and develop a model for wind generated noise from a hurricane for both single sensors and arrays. We use this model to demonstrate the potential usefulness of quantifying hurricane wind speed with underwater acoustic sensors. 


\subsection{Hurricane Structure and Current Classifica- tion Techniques}

Hurricanes are severe storms characterized by surface winds from 33 to over $80 \mathrm{~m} / \mathrm{s}$ [33] that circulate around a central low pressure zone called the eye. Holland[52] gives an analytic model for the surface wind speed profile as a function of range from the eye since hurricanes are typically cylindrically symmetric,

$$
V=\sqrt{A B\left(p_{n}-p_{c}\right) \frac{\exp \left\{\frac{-A}{r^{B}}\right\}}{\rho_{a} r^{B}}}
$$

where $V$ is wind speed at a height of $10 \mathrm{~m}$ above the sea surface, $p_{c}$ and $p_{n}$ are the atmospheric pressure in the eye and outside the hurricane respectively, $\rho_{a}$ is the density of the air, and $A$ and $B$ are empirical values. Using this model, the surface wind speed profile for a moderate hurricane is given in Fig. 2-1, where wind speed in the eye is zero and rapidly increases to a maximum of $50 \mathrm{~m} / \mathrm{s}$ at what is known as the eye wall. Outside of the eye wall, which is on the order of ten kilometers thick, wind speed slowly decreases to the edge of the hurricane which is typically hundreds of kilometers from the eye. Most of a hurricane's destructive power then comes from the high winds in the eye wall since this power is roughly proportional to the cube of the maximum wind speed.[53]

The standard approach for classifying a hurricane's destructive power, the Dvorak method, $, 31,32,131]$ is effectively a pattern-recognition technique where satellite images, in the visible and infra-red spectrum, are used to classify the hurricane based on features like the size and the geometry of cloud patterns. As discussed in Chapter 1 , this method often yielded wind speed estimates with errors of over $40 \%$ in several recent hurricanes.[93, 43, 12, 4, 121] Despite these errors, the Dvorak method is still the primary technique for classifying the destructive power of a hurricane from satellite measurements.[44] A satellite-based pattern-recognition technique similar to 


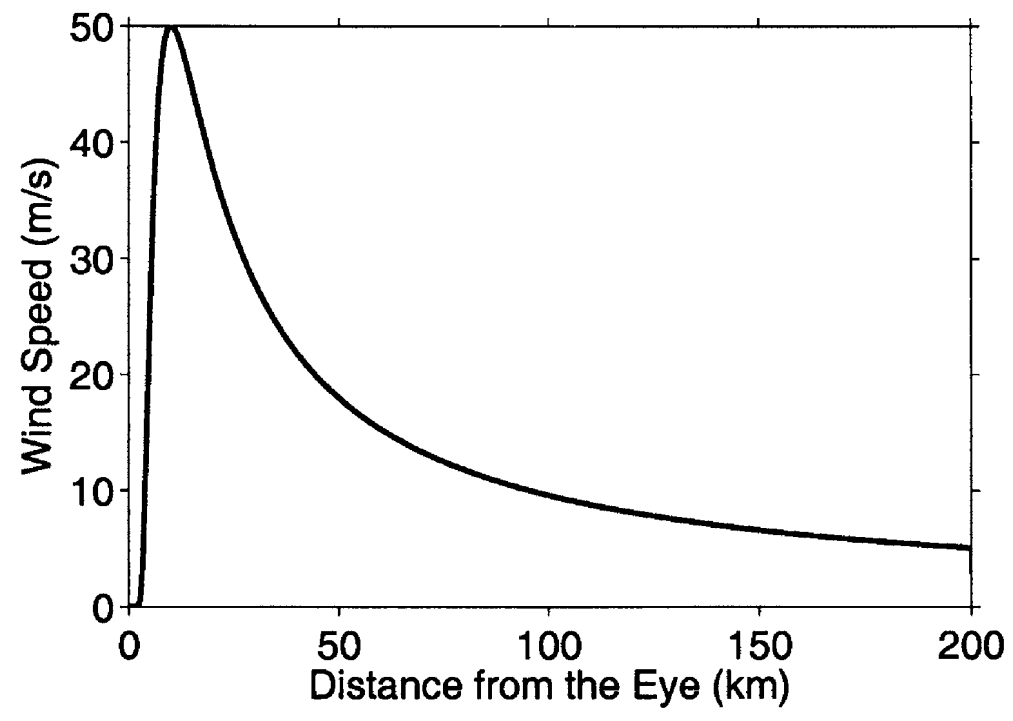

Figure 2-1: Hurricane wind speed as a function of distance from the hurricane center based on Holland's model[52] with parameters $A=72.44, B=1.86, p_{c}=96300 \mathrm{~Pa}$, $p_{n}=100500 \mathrm{~Pa}$ and $\rho_{A I R}=1.15 \mathrm{~kg} / \mathrm{m}^{3}$. The zero wind speed region at the center of the hurricane $(0 \mathrm{~km})$ is called the eye and the high wind speed region $(10 \mathrm{~km})$ is the eye wall. The total destructive power of the hurricane is proportional to the cube of the maximum wind speed, which occurs in the eye wall.[53] 
the Dvorak method using SSM/I satellite microwave $(85 \mathrm{GHz})$ instead of optical and infrared images has recently been developed but gives similar errors as the Dvorak method.[7]

Satellite classification of hurricanes with Microwave Sounding Units (MSU)[130] is secondary to the primary Dvorak method[44] due to the limited spatial resolution of the unit. The $55 \mathrm{GHz}$ microwave radiation given off by warm air in the hurricane's eye is used to estimate temperature and then infer the hurricane's power. Because of the small size of the satellite array unit the spatial resolution of the measurement is about $48 \mathrm{~km}$,[65] which is often larger than the diameter of the eye, resulting in a blurred image of the hurricane and potentially leading to errors in the estimate of the destructive power. $[130,65]$

Other satellite techniques for estimating hurricane wind speed and destructive power are currently being studied. For an overview see the article by Katsaros, Vachon, Liu and Black.[59] These techniques, however, are still under development and are not yet being used operationally for hurricane classification and disaster planning.[41]

To overcome the limitations of satellite techniques, specially equipped aircraft, like the Air Force's WC-130s and NOAA's WP-3s, are flown through the center of a hurricane.[41] Using on-board sensors and expendable dropsondes, accurate wind speed estimates with errors less than $5 \mathrm{~m} / \mathrm{s}$ can be obtained.[41] Unfortunately, as discussed in the introduction, these aircraft are expensive to purchase and operate and are currently only used by the United States.[33] 


\subsection{Wind Generated Surface Noise from Uncorre- lated Surface Sources}

Here we develop a model for the surface generated noise intensity and mutual intensity from a hurricane received by a hydrophone or hydrophone array submerged in an ocean waveguide. The geometry of the problem is shown in Fig. 2-2. The hurricane is centered at the origin and is surrounded by ambient winds all of which cause local sea-surface agitation. This agitation leads to sound sources with amplitude dependent on the local wind speed that are modeled as a sheet of monopoles on a source plane at a depth $z_{0}$ within a quarter wavelength of the free surface following ocean acoustic noise modeling convention.[70, 19, 95] Intensity and mutual intensity are determined by directly integrating the surface source contributions using the waveguide Green function.

Several previous authors have addressed similar surface noise problems, however, their derivations are intertwined with approximations or parameterizations that are not suitable for modeling hurricane noise. Kuperman and Ingenito[70] developed a widely used surface noise model, however, embedded in their derivation is the assumption that the source field is range independent. This is not true for hurricane generated noise where the wind speed and source level change drastically with position.

Using an adiabatic normal mode formulation Perkins, Kuperman, Ingenito, Fialkowski, and Glattetre[95] extended the model of Kuperman and Ingenito to rangedependent source fields and mildly range-dependent waveguides. They did this by dividing the surface area into smaller sub-areas over which the source field could be considered constant. They used far-field approximations for each sub-area. These were coupled with the further approximation that the cross-spectral density for each sub-area could be expressed as a single sum over modes. This approximation is only 


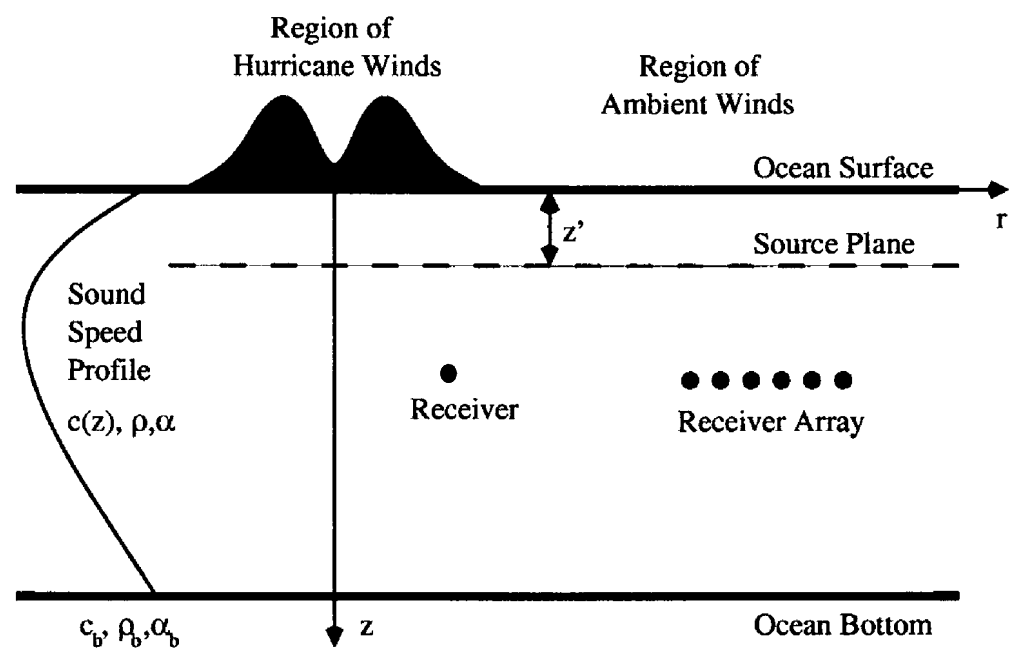

Figure 2-2: Cross section of the stratified ocean waveguide showing the geometry of the surface noise problem (Not to scale). On the surface is the area covered by the hurricane and surrounding area covered $5 \mathrm{~m} / \mathrm{s}$ ambient winds. The surface noise sources are modeled as a plane of monopoles a small depth $z_{0}$ below the surface and the sound field is measured by receivers and receiver arrays. 
valid when the inverse of the difference between the horizontal wavenumber of the modes is much less than the dimension of the sub-area.[108, 109] For the highly rangedependent winds of a hurricane in an otherwise range-independent waveguide, this approach proves to be less accurate, more cumbersome and less efficient to implement than direct integration.[140] Carey, Evans, Davis and Botseas[19] have developed a computational approach based on the parabolic equation approximation for calculating range-dependent surface noise. We find that steep angle contributions dominate the intensity measured by a single sensor and so require direct integration of local noise sources with a full-field model for the Green function rather than an elevationangle-restricted parabolic approximation.

It is useful to briefly derive the direct integration approach used here since it has not explicitly appeared in the previous literature even though many essential elements are implicit in the work of Perkins, Kuperman, Ingenito, Fialkowski and Glattetre.[95] For uncorrelated sources the cross-spectral density of the noise field can be written as

$$
C\left(\mathbf{r}_{\mathbf{1}}, \mathbf{r}_{\mathbf{2}}, f\right)=\int_{\infty} d^{2} \boldsymbol{\rho}_{\mathbf{0}} \frac{S_{q q}\left(V\left(\boldsymbol{\rho}_{0}\right), f\right)}{\Delta A} g\left(\mathbf{r}_{\mathbf{1}} \mid \mathbf{r}_{\mathbf{0}}, f\right) g^{*}\left(\mathbf{r}_{\mathbf{2}} \mid \mathbf{r}_{\mathbf{0}}, f\right)
$$

as shown in Appendix A where $S_{q q}\left(V\left(\rho_{0}\right), f\right)$ is the source power-spectral density, which is a function of wind speed $V$ and frequency $f, \Delta A$ is a small area increment of integration at least the size of the horizontal coherence area of the source distribution, and $g\left(\mathbf{r}_{\mathbf{j}} \mid \mathbf{r}_{\mathbf{0}}, f\right)$ is the waveguide Green function. Throughout this paper a cylindrical coordinate system in used where $\mathbf{r}=(\boldsymbol{\rho}, z)=(\rho, \theta, z), \boldsymbol{\rho}$ is the horizontal location vector, $\rho$ is distance from the origin, $\theta$ is azimuth angle and $z$ is depth measured with positive downward from the surface. The locations $\mathbf{r}_{1}$ and $\mathbf{r}_{2}$ are receivers and $\mathbf{r}_{\mathbf{0}}$ is the source. Green functions are calculated by a combination of wavenumber integration at short ranges and the normal mode approximation at long ranges. The integration over surface source area is computed numerically. This expression is valid 
for range-dependent source fields and environments.

The source depth $z_{0}$ is taken to be a quarter wavelength for all simulations in the present paper. This follows noise modeling convention[70, 19, 95] since source depths of a quarter wavelength or less lead to a downward-directed dipole source radiation pattern. Hamson has shown that on average wind generated noise in the ocean radiates with a downward directed pattern that closely fits a dipole for wind speeds between 5 and $20 \mathrm{~m} / \mathrm{s}$ and frequencies from $400 \mathrm{~Hz}$ to $3.2 \mathrm{kHz}$. [50] This is true even for average source depths greater than a quarter wavelength and sea-surface roughness much larger than the wavelength $[50,101]$ as in a hurricane where wave heights may exceed $10 \mathrm{~m}$. This is understandable since surface noise is believed to arise from many monopole sources, in particular bubbles, randomly distributed near the sea surface. All of these, by the method of images, have main downward directed lobes which reinforce and varying horizontally directed side-lobes which cancel.

As discussed in the introduction, the source power-spectral density has been shown to follow

$$
S_{q q}(V, f)=s_{0}(f) V^{n(f)}
$$

for certain frequency and wind speed ranges. While experiments[102] at wind speeds below $20 \mathrm{~m} / \mathrm{s}$ give $n=3.1 \pm 0.3$, values in the broader $n=1$ to $n=4$ range will be used here for illustrative purposes. If it is later found that wind speed and noise intensity are related by some other function, the power-law relationships considered here will provide a basis for piecewise construction of this more complicated dependence.

Farmer has shown experimentally that clouds of bubbles near the ocean surface may, through scattering and absorption, lower ambient noise levels at frequencies above $8 \mathrm{kHz}$ and wind speeds above $15 \mathrm{~m} / \mathrm{s}$.[40] While such attenuation has never been observed at lower frequencies, we will consider its possibility in the high winds of a hurricane. 
Attenuation, in $\mathrm{dB} / \mathrm{m}$, can be written as $\alpha=10 \log (e) \sigma n_{v}$, where $\sigma$ is the extinction cross section of an individual bubble and $n_{v}$ is the number of bubbles per unit volume.[139] Using this expression, Weston[139] provides a model for attenuation by sea surface bubble clouds, based on the extinction cross section and spatial distribution of wind-generated bubbles as a function of wind speed and frequency. This attenuation can then be included in the Green function in Eq. 2.2 to determine its effect on the underwater noise field. This is done by calculating the Green function for a waveguide with an effective attenuation in $\mathrm{dB} / \mathrm{m}$ of

$$
\alpha(V, f)=\left\{\begin{aligned}
9.35 \times 10^{-7} \sqrt{f} V^{3} & : f<1.5 k H z \\
2.44 \times 10^{-8} f V^{3} & : \quad f>1.5 k H z
\end{aligned}\right.
$$

in a layer at the sea surface as given by Weston.[139]

\subsection{Single Hydrophone Analysis}

Here it is shown that the noise intensity measured by a single sensor in a hurricane is dominated by local sea-surface sources rather than sound propagating from longer ranges. Underwater acoustic intensity can then be used to estimate the wind speed within a local resolution area since wind speed in a hurricane is also found to be effectively constant over this scale.

Beginning with the cross-spectral density of the noise field in a hurricane, Eq. (2.2), the spectral intensity of the sound field received at $\mathbf{r}$ can written as

$$
I(\mathbf{r}, f)=\frac{C(\mathbf{r}, \mathbf{r}, f)}{\rho_{w} c}=\int_{-\infty}^{\infty} d^{2} \boldsymbol{\rho}_{0} \frac{S_{q q}\left(V\left(\boldsymbol{\rho}_{0}\right), f\right)}{\rho_{w} c \Delta A}\left|g\left(\mathbf{r} \mid \mathbf{r}_{\mathbf{0}}, f, V\left(\boldsymbol{\rho}_{\mathbf{0}}\right)\right)\right|^{2}
$$

where the total instantaneous intensity is given by 


$$
I(\mathbf{r})=\int_{0}^{\infty} I(\mathbf{r}, f) d f
$$

The Green function $g\left(\mathbf{r} \mid \mathbf{r}_{\mathbf{0}}, f, V\left(\boldsymbol{\rho}_{\mathbf{0}}\right)\right)$ depends on local wind speed $V\left(\boldsymbol{\rho}_{\mathbf{0}}\right)$ because it includes attenuation due to wind-generated sea-surface bubbles. We show that this wind speed dependence is negligible at frequencies less than $100 \mathrm{~Hz}$ for typical hurricane wind speeds, but needs to be accounted for at higher frequencies. Surface wind speed $V$ is given by the Holland model of Fig. 2-1 for a hurricane, while the surrounding ambient wind speed is taken to be $5 \mathrm{~m} / \mathrm{s}$.

Two hurricane-prone ocean environments surrounded by densely populated coastal communities, the North Atlantic and the Bay of Bengal, are considered. Their sound speed profiles are shown in Fig. 2-3. The difference in water depth between these two environments leads to fundamental differences in propagation. Typical near-surface sound sources will lead to refractive propagation with excess depth in the North Atlantic but not in the Bay of Bengal. In the former, sound may propagate efficiently to long ranges via the deep-sound channel, while in the latter, it will multiply reflect off the lossy bottom leading to far greater transmission loss. Although hurricanes decrease the temperature of the local sea surface by roughly $1^{\circ} \mathrm{C}$ near the eye wall to roughly $35 \mathrm{~m}$ depth, the corresponding small change in sound speed[55] of roughly $4 \mathrm{~m} / \mathrm{s}$ is also local and so has a negligible effect on the curvature of both local and long-range sound paths.

The spectral intensity level, given by

$$
L_{I}=10 \log \left(\frac{I(\mathbf{r}, f)}{I_{\text {ref }}(f)}\right)
$$

in $\mathrm{dB}$ re $I_{\text {ref }}(f)$, of hurricane generated noise is computed by the direct integration of Eq. 2.5 as a function of receiver range $\rho$ and depth $z$ from an origin at the center of the hurricane on the sea-surface. For convenience in the present paper the reference 


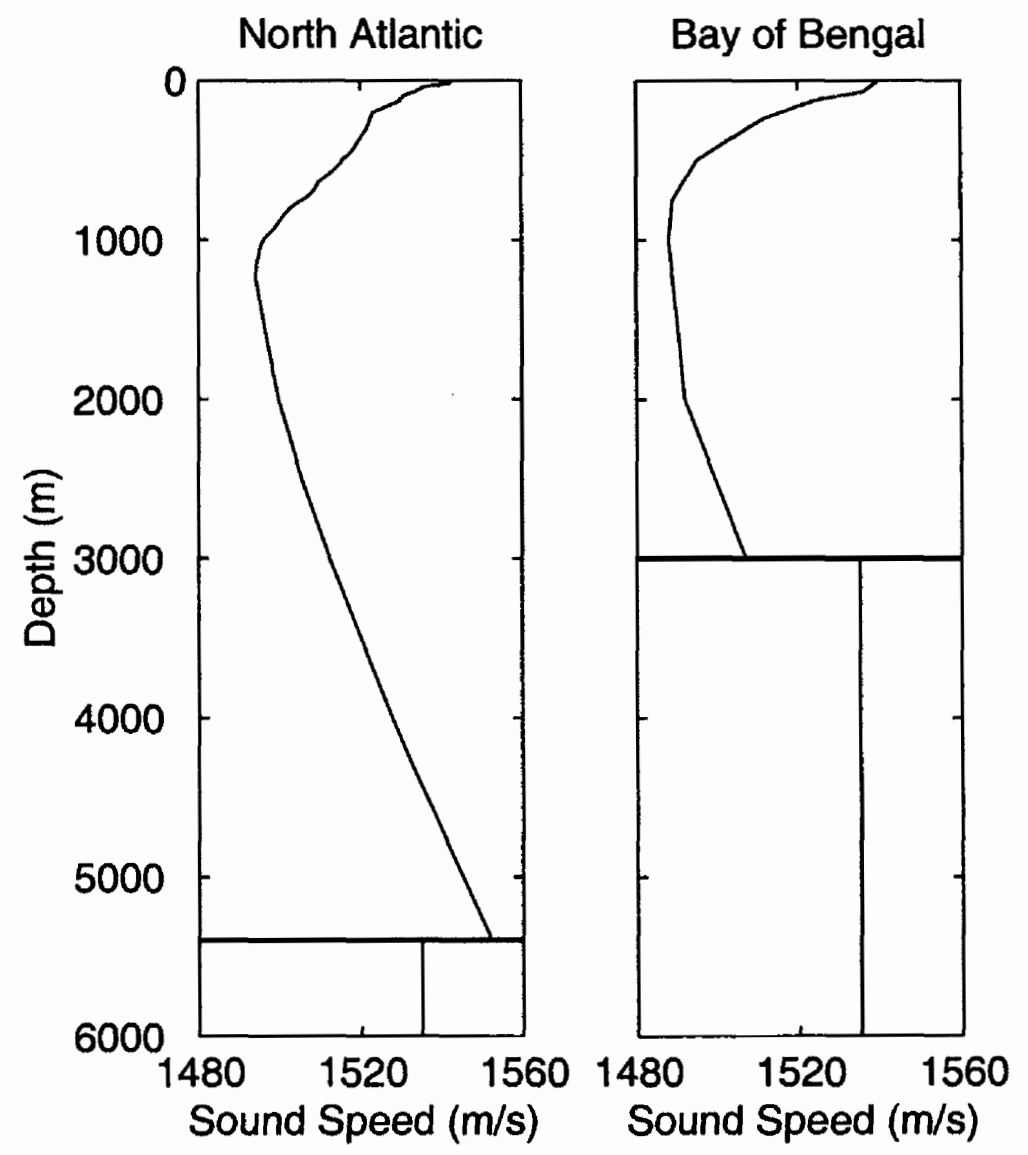

Figure 2-3: Sound speed profiles $c(z)$ for the North Atlantic[82] and the Bay of Bengal $[69,114]$. The bottom has a density of $1.38 \mathrm{~g} / \mathrm{cm}$ and an attenuation of $0.3 \mathrm{~dB} / \lambda$ corresponding to the deep silty sediment layers of the Bay of Bengal[123, 48] and the North Atlantic Abyssal plain[49, 125]. The water has a density of $1 \mathrm{~g} / \mathrm{cm}$ and an attenuation of $6 \times 10^{-5} \mathrm{~dB} / \lambda$. 
level $I_{r e f}(f)$ is taken to be the spectral intensity at a reference depth $z_{r e f}=200 \mathrm{~m}$ for a reference $10-\mathrm{m}$ altitude wind speed of $V_{r e f}=5 \mathrm{~m} / \mathrm{s}$ over the entire ocean

$$
I_{r e f}(f)=I\left(\mathbf{r}_{r e f}, f\right)=\int_{-\infty}^{\infty} d^{2} \rho_{0} \frac{S_{q q}\left(V_{r e f}, f\right)}{\rho_{w} c \Delta A}\left|g\left(\mathbf{r}_{r e f} \mid \mathbf{r}_{\mathbf{0}}, f, V_{r e f}\right)\right|^{2}
$$

where $\mathbf{r}_{r e f}=\left(\boldsymbol{\rho}, z_{r e f}\right)$. Noise intensity has been measured for $5 \mathrm{~m} / \mathrm{s}$ wind speed in many ocean environments and at similar depths.[102, 24, 97, 20] In an experimental scenario other reference values could be chosen.

Spectral intensity level is shown in Fig. 2-4 for frequencies of 50, 400 and $3200 \mathrm{~Hz}$, spaced three octaves apart, using Eqs. (2.3)-(2.8) and assuming $n=3$. The choice of $n=3$ is within measured power-laws[102] and has been chosen out of convenience since it is linearly related to the power of the wind.[34] The wind speed profile of the hurricane and surroundings based on the Holland model at an altitude of $10 \mathrm{~m}$ from the sea surface is also plotted with the spectral intensity level at a depth of $200 \mathrm{~m}$. The most apparent feature in Fig. 2-4(a) and (c) is the effectively linear relationship at low frequency, $50 \mathrm{~Hz}$, between spectral intensity level $L_{I}$ and the log of the wind speed. This is roughly independent of depth as can be seen in Figs. 2-4(b) and (d). At the higher frequencies shown sea-surface bubbles significantly attenuate sound in the high wind speed, eye-wall region of the hurricane but the noise still follows local wind speed with a more complicated nonlinear dependence as will be shown in the next section. The small increase in level in the North Atlantic outside the hurricane at ranges of 193 and $257 \mathrm{~km}$ and at a depth of $4.7 \mathrm{~km}$ is caused by convergence zone propagation from the powerful sources in the eye wall. This convergence zone structure indicates an efficient mechanism exists for the long range propagation of hurricane noise in this environment that will be considered in Sec. 2.5 . 

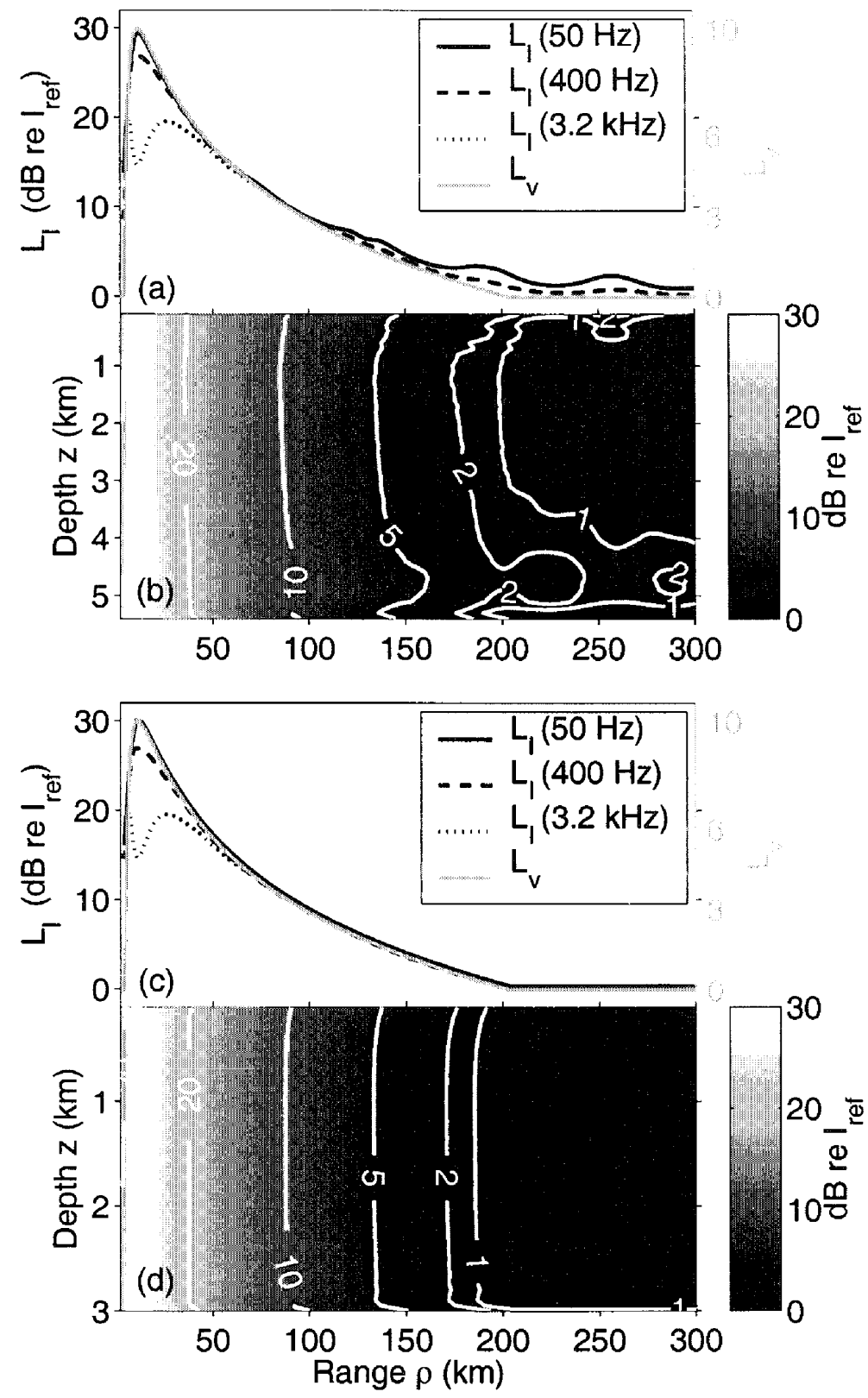

Figure 2-4: Noise spectral level ( $\mathrm{dB}$ re $I_{\text {ref }}$ ) in the North Atlantic (a) and (b) and the Bay of Bengal (c) and (d) for $n=3$. (a) and (c) show the level as a function of range at a depth of $200 \mathrm{~m}$ for 50,400 and $3200 \mathrm{~Hz}$ frequencies. $L_{V}(\boldsymbol{\rho})=10 \log \left(V(\boldsymbol{\rho}) / V_{\text {ref }}\right)$ is plotted for comparison where $V_{\text {ref }}=5 \mathrm{~m} / \mathrm{s}$. $L_{V}=0$ is equivalent to $V=5 \mathrm{~m} / \mathrm{s}$ and $L_{V}=10$ is equivalent to $V=50 \mathrm{~m} / \mathrm{s}$. (b) and (d) show the level as a function of range and depth at $50 \mathrm{~Hz}$. In both waveguide environments the noise level closely follows the local wind speed. In the North Atlantic there is a convergence zone structure corresponding to sound that propagates from the hurricane's eye wall via refraction. Note the convergence zone near the surface at a range of $257 \mathrm{~km}$ and the ray vertex depth of $4.7 \mathrm{~km}$. 


\subsubsection{Local Noise Dominates}

The effectively linear relationship between the $\log$ of the local wind speed and underwater acoustic spectral intensity shown in Fig. 2-4 suggests a possible simplifying approximation to our formulation. In particular the areal integral of Eq. (2.5) can be approximated by integrating only over the local sources in the hurricane. These fall within a disc of area $A=\pi R^{2}$ centered at the horizontal location of the receiver $\rho$ which provides the dominant contribution in the exact integral (Eq. (2.5)). The spectral intensity can then be approximated as

$$
I(\mathbf{r}, f) \approx \int_{A} d^{2} \boldsymbol{\rho}_{\mathbf{0}}^{\prime} \frac{S_{q q}\left(V\left(\boldsymbol{\rho}_{0}^{\prime}\right), f\right)}{\rho_{w} c \Delta A}\left|g\left(\mathbf{r} \mid \mathbf{r}_{\mathbf{0}}, f, V\left(\boldsymbol{\rho}_{\mathbf{0}}{ }^{\prime}\right)\right)\right|^{2}
$$

where $\rho_{0}^{\prime}=\rho_{0}-\rho$. Such a simplification can potentially lead to errors if $R$ is too small.

To quantify the potential error of this local approximation, the approximate equation (2.9) is evaluated for a receiver under the eye wall of the hurricane where wind speed varies most drastically. When compared to the exact result of Eq. (2.5), we take the error induced by the local approximation to be negligible, less than or equal to $1 \mathrm{~dB}$, for $R$ greater than a minimum length $R_{\text {local }}$. The error as a function of $R$ is given in Fig. 2-5 where, for deep-water environments, $R_{\text {local }}=300$ to $2000 \mathrm{~m}$ depending on sensor depth.

It is noteworthy that the deep-ocean North Atlantic and Bay of Bengal error curves closely match those of the infinite half-space. This shows that bottom reflections and variations in sound speed profile do not have a significant effect on $R_{\text {local }}$ in deep water. For a bottom mounted sensor in a typical shallow water environment $R_{\text {local }}=2$ to $3 \mathrm{~km}$ in the 50 to $400 \mathrm{~Hz}$ range. Our computations also show that $R_{\text {local }}$ does not change significantly for the expected source power-spectral densities and attenuations 

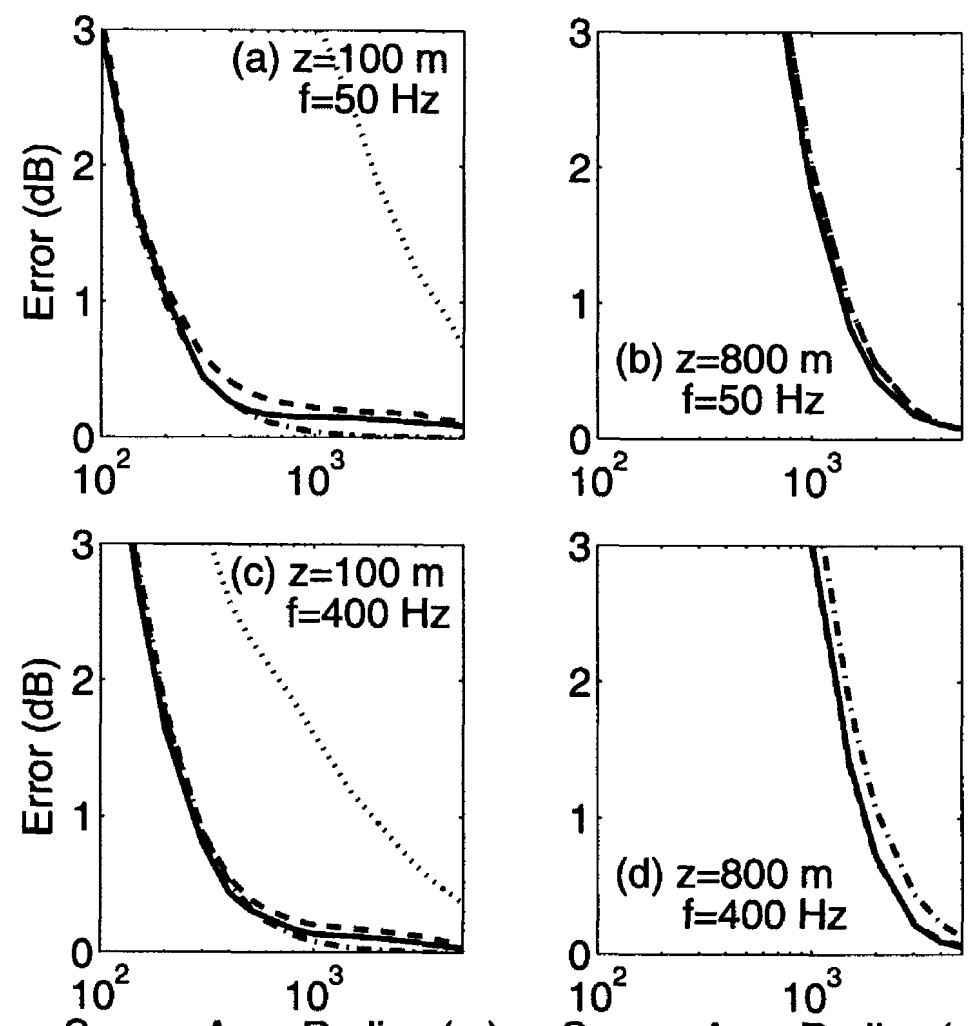

Source Area Radius (m)

Source Area Radius (m)

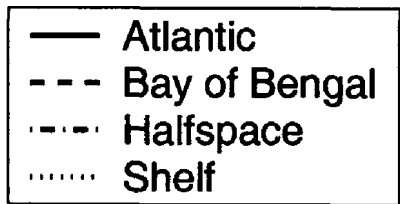

Figure 2-5: Error induced by the local area approximation (Eq. (2.9)) as a function of local source area radius $R_{\text {local }}$ for a single sensor under the maximum winds in the eye wall of a hurricane. Curves are shown for the North Atlantic and the Bay of Bengal environments used in this paper as well as for infinite half-space and shallow water continental shelf environments. Plots are given for sensor depths of $100 \mathrm{~m}$ ((a) and (c)) and $800 \mathrm{~m} \mathrm{((b)} \mathrm{and} \mathrm{(d))} \mathrm{and} \mathrm{for} \mathrm{frequencies} \mathrm{of} 50 \mathrm{~Hz}((\mathrm{a})$ and (b)) and 400 $\mathrm{Hz}((\mathrm{c})$ and $(\mathrm{d}))$. While these plots are given for $n=3$ the difference for values $n=1$ to 4 is less than $0.1 \mathrm{~dB}$. The North Atlantic, Bay of Bengal and infinite half-space environments are very similar. In these deep-water environments, for the shallow $100 \mathrm{~m}$ sensor depth, we see that for $R_{\text {local }}$ over roughly $300 \mathrm{~m}$ the error from this approximation is negligible. For the deeper $800 \mathrm{~m}$ sensor depth, the $R_{\text {local }}$ for which the error is negligible is roughly $2 \mathrm{~km}$. In shallow water the error in the local area approximation is higher leading to a larger $R_{\text {local }}$. This is likely due to the strong reflection of sound off bottom. In deep water environments bottom reflections have little effect and most of the sound measured by a receiver propagates via direct path from the surface source. 
considered in this paper.

The wind speeds in a hurricane do not change significantly over $R_{\text {local }}$ and can be approximated as constant in Eq. (2.9). This leads to less than $0.2 \mathrm{~dB}$ additional error in the spectral intensity level, which can then be approximated as

$$
\begin{aligned}
I(\mathbf{r}, f) & \approx \frac{S_{q q}(V(\boldsymbol{\rho}), f)}{\rho_{w} c \Delta A} \int_{0}^{2 \pi} \int_{0}^{R_{l o c a l}} \rho_{0}^{\prime} d \rho_{0}^{\prime} d \theta_{0}^{\prime}\left|g\left(\mathbf{r} \mid \mathbf{r}_{\mathbf{0}}, f, V(\boldsymbol{\rho})\right)\right|^{2} \\
& \equiv S_{q q}(V(\boldsymbol{\rho}), f) W(\mathbf{r}, f, V(\boldsymbol{\rho}))
\end{aligned}
$$

where only the local wind speed $V(\boldsymbol{\rho})$ directly above the receiver has a significant effect on both the source factor $S_{q q}(V(\boldsymbol{\rho}), f)$ and the waveguide propagation factor $W(\mathbf{r}, f, V(\boldsymbol{\rho}))$. The source factor is universal in that it does not depend on propagation parameters and should be the same for any waveguide environment so long as the ocean depth greatly exceeds the ocean-atmosphere boundary layer. While the propagation factor does depend on the environment, ocean waveguides typically change gradually with horizontal position. The wind-speed-independent functionality of $W(\mathbf{r}, f, V(\boldsymbol{\rho}))$ should then be effectively constant over $R_{\text {local }}$ and over the horizontal extent of a hurricane, on the order of $100 \mathrm{~km}$. Both factors may be characterized numerically or empirically to develop a set of curves to estimate wind speed from acoustic intensity. In the next section we find that it is possible to simplify these factors and develop an approximate analytic equation for wind speed estimation.

The approximate Eq. (2.10) for range-dependent sources and potentially rangedependent waveguides is similar to Kuperman and Ingenito's[70] exact Eq. (30) for range-independent sources and waveguides in that spectral intensity is the product of a "universal ambient noise" source factor, following Ingenito and Wolf[57] and here defined as $S_{q q}(V(\boldsymbol{\rho}), f)$, and a waveguide propagation factor $W(\mathbf{r}, f, V(\boldsymbol{\rho}))$. The implicit assumption of formulations of this kind is that variations in source depth can 
be accounted for as equivalent variations in $S_{q q}(V(\boldsymbol{\rho}), f)$. This is consistent with the measured dipole behavior of ambient noise in the ocean.[50]

Taking the $\log$ of Eq. (2.10) leads to a useful approximate equation for spectral intensity level

$$
L_{I}(\mathbf{r}, f) \approx L_{S}(V(\boldsymbol{\rho}), f)+L_{W}(\mathbf{r}, f, V(\boldsymbol{\rho}))
$$

in $\mathrm{dB}$ re $I_{r e f}(f)$ where

$$
\begin{gathered}
L_{S}(V(\boldsymbol{\rho}), f)=10 \log \left(\frac{S_{q q}(V(\boldsymbol{\rho}), f)}{S_{q q}\left(V_{\text {ref }}, f\right)}\right) \\
L_{W}(\mathbf{r}, f, V(\boldsymbol{\rho}))=10 \log \left(\frac{W(\mathbf{r}, f, V(\boldsymbol{\rho}))}{W_{\text {ref }}(f)}\right)
\end{gathered}
$$

and

$$
I_{r e f}(f)=S_{q q}\left(V_{r e f}, f\right) W\left(\mathbf{r}_{r e f}, f, V_{r e f}\right)=S_{q q}\left(V_{r e f}, f\right) W_{r e f}(f)
$$

Here $L_{S}(V(\boldsymbol{\rho}), f)$ is a universal ambient noise source term that is independent of waveguide propagation parameters, while $L_{W}(\mathbf{r}, f, V(\boldsymbol{\rho}))$ is a waveguide propagation term. The functional dependencies of the first term can be determined empirically in any waveguide where the ocean depth greatly exceeds the ocean-atmosphere boundary layer, while the functional dependencies of the second term should be locally determined.

If $S_{q q}(V(\boldsymbol{\rho}), f)$ follows a power-law, such as Eq. (2.3), then universal ambient noise source level is linearly related by 


$$
L_{S}(V(\boldsymbol{\rho}), f)=10 n(f) \log \left(\frac{V(\boldsymbol{\rho})}{V_{r e f}}\right)
$$

to the $\log$ of wind speed. The slope of this linear relationship $10 n(f)$ has been previously measured in the $13 \mathrm{~Hz}$ to $14.5 \mathrm{kHz}$ frequency range and 1 to $20 \mathrm{~m} / \mathrm{s}$ wind speed range.[118, 38, 102, 24, 20]

To estimate wind speed from ambient noise measurements using Eq. (2.11), the dependence of $L_{W}(\mathbf{r}, f, V(\boldsymbol{\rho}))$ on wind-dependent attenuation by sea-surface bubbles needs to be established. This may be done empirically, numerically or analytically as in the next section.

\subsubsection{Separating the Effect of Attenuation by Bubbles from Local Waveguide Propagation}

Analytic expressions are derived to show how attenuation can be separated from other waveguide propagation effects so that $L_{W}(\mathbf{r}, f, V(\boldsymbol{\rho}))$ can be split into a universal wind-speed-dependent attenuation term and a local waveguide calibration term that is wind-speed independent. These analytic expressions also demonstrate the uniqueness of a wind speed estimate based on broad-band underwater noise measurements. They also enable analytic expressions for estimation error to be obtained in some important cases.

Underwater spectral intensity level is calculated over a range of wind speeds and frequencies relevant to hurricane quantification as illustrated in Fig. 2-6 using the full areal integration of Eq. (2.5). The spectral intensity level exhibits a maxima that depends on wind speed and frequency. For wind speeds and frequencies below this maxima, attenuation by bubbles is negligible so that $L_{W}(\mathbf{r}, f, V(\boldsymbol{\rho}))$ is only a function of the local waveguide environment and spectral intensity level $L_{I}(\mathbf{r}, f)$ should 
depend on the log of wind speed only through Eq. (2.15) given the power-law $n=3$ assumption of the simulation. For higher wind speeds and frequencies, attenuation by bubbles is significant and eventually leads to a roll-off in the spectral intensity so that $L_{W}(\mathbf{r}, f, V(\boldsymbol{\rho}))$ is a separable function of both wind-speed-dependent and wind-speed-independent terms.

While the dependence of spectral intensity on wind speed and frequency including attenuation by bubbles can be calculated exactly using the full areal integration of Eq. (2.5) or the local integral approximations of Eqs. (2.9) or (2.10), a useful first-order approximation leads to the analytic result

$$
W(\mathbf{r}, f, V(\boldsymbol{\rho}))=W_{0}(\mathbf{r}, f) \frac{4 \pi^{2}\left|A\left(V(\boldsymbol{\rho}), f, k_{r}=0\right)\right|^{2}}{z_{0}^{2}}
$$

where

$$
W_{0}(\mathbf{r}, f)=W(\mathbf{r}, f, 0)
$$

and

$$
A\left(V(\boldsymbol{\rho}), f, k_{r}=0\right)=\frac{\sin \left(k z_{0}\right)}{2 i \pi \frac{\alpha}{20 \log (e)} \cos (k L)+\frac{2 \pi \omega}{c\left(z_{0}\right)} e^{-i k L}}
$$

is the downward plane-wave amplitude for a source in an attenuating sea-surface bubble layer following the Pekeris solution. [58] The complex wavenumber $k=\frac{\omega}{c\left(z_{0}\right)}+$ $i \frac{\alpha(V(\boldsymbol{\rho}), f)}{20 \log (e)}$ is used in Eq. (2.18) where $\alpha(V(\boldsymbol{\rho}), f)$ is given in Eq. (2.4).

The spectral intensity level of Eq. (2.11) can then be approximated as

$$
L_{I}(\mathbf{r}, f) \approx L_{S}(V(\boldsymbol{\rho}), f)+L_{A}(V(\boldsymbol{\rho}), f)+L_{W_{0}}(\mathbf{r}, f)
$$

where 


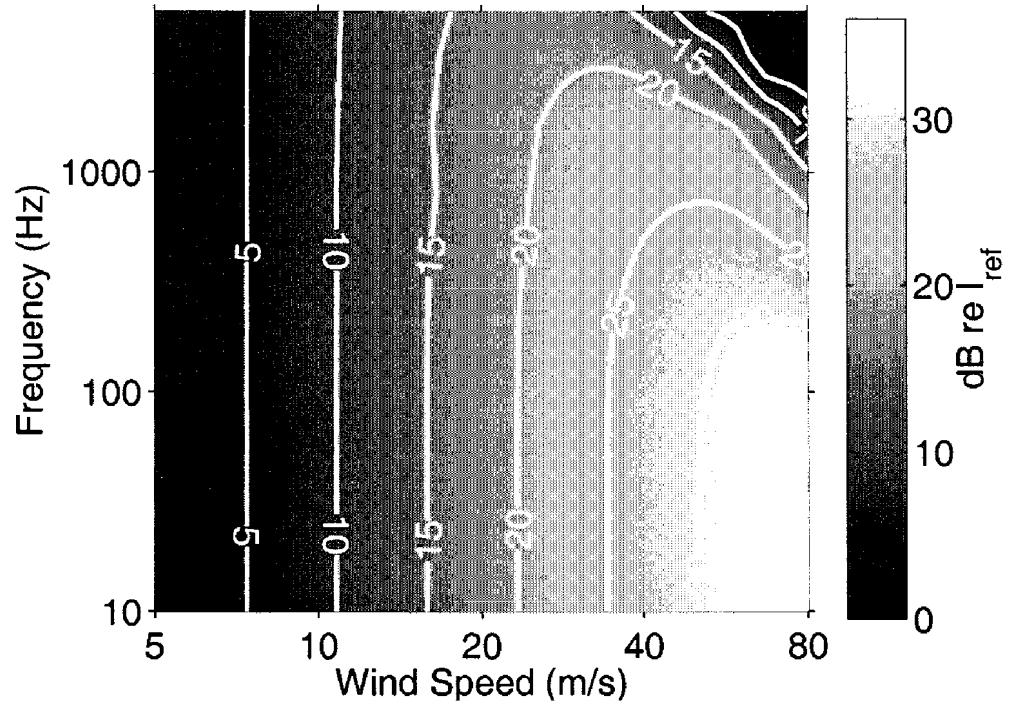

Figure 2-6: Simulated noise spectral level (dB re $I_{r e f}$ ) in the North Atlantic for range-independent winds as a function of wind speed and frequency including attenuation by sea-surface bubbles assuming $n=3$ from Eq. (2.5). Below $100 \mathrm{~Hz}$ the power-law relationship between noise intensity and wind speed is unaffected by bubble attenuation even up to the $80 \mathrm{~m} / \mathrm{s}$ wind speeds of a hurricane. As frequency increases, attenuation affects the noise level at progressively lower wind speeds. For a given frequency the noise level increases linearly with wind speed, peaks, and then decays exponentially. 


$$
L_{A}(V(\boldsymbol{\rho}), f)=20 \log \left(\frac{2 \pi\left|A\left(V(\boldsymbol{\rho}), f, k_{r}=0\right)\right|}{z_{0}}\right)
$$

and

$$
L_{W_{0}}(\mathbf{r}, f)=10 \log \left(\frac{W_{0}(\mathbf{r}, f)}{W_{r e f}(f)}\right)
$$

The approximation of Eq. (2.19) is in agreement with the full areal integration of Eq. (2.5) to within $1 \mathrm{~dB}$ for frequencies below $500 \mathrm{~Hz}$ even at hurricane wind speeds as shown in Fig. 2-7.

By splitting the local waveguide and bubble attenuation effects of $L_{W}(\mathbf{r}, f, V(\boldsymbol{\rho}))$ into two terms, $L_{A}(V(\boldsymbol{\rho}), f)$ and $L_{W_{0}}(\mathbf{r}, f)$, wind speed can now be estimated from ambient noise using Eq. (2.19), where $L_{A}(V(\boldsymbol{\rho}), f)$ is a universal attenuation term that depends on local wind speed but like $L_{S}(V(\rho), f)$ is also independent of waveguide parameters. The last term of Eq. (2.19), $L_{W_{0}}(\mathbf{r}, f)$, is a local waveguide calibration that is independent of wind speed.

At frequencies below $100 \mathrm{~Hz}$ where attenuation $\alpha$ due to bubbles is negligible at hurricane wind speeds $L_{A}(V(\rho), f)$ goes to zero, as expected from Fig 2-6. In this important case, if $S_{q q}(V(\boldsymbol{\rho}), f)$ follows a power law, Eq. (2.19) reduces to a linear equation in the log of wind speed

$$
L_{I}(\mathbf{r}, f) \approx 10 n(f) \log \left(\frac{V(\boldsymbol{\rho})}{V_{\text {ref }}}\right)+L_{W_{0}}(\mathbf{r}, f)
$$

where $10 n(f)$ is a universal empirically determined slope and $L_{W_{0}}(\mathbf{r}, f)$ is a local calibration intercept. The log of wind speed can be then found from measurements 


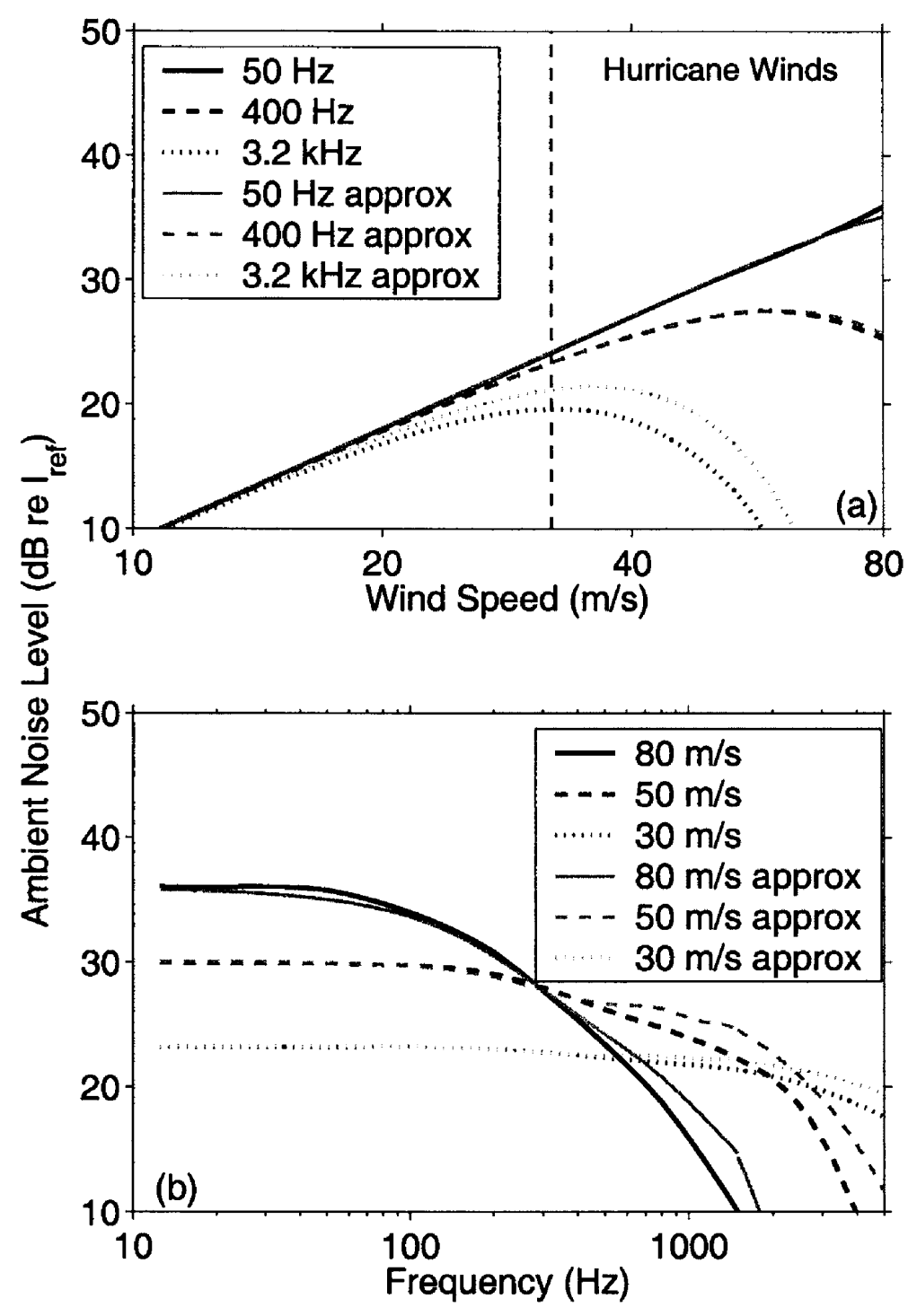

Figure 2-7: (a) Noise spectral level ( $\mathrm{dB}$ re $I_{\text {ref }}$ ) as a function of wind speed at several frequencies, assuming $n=3$. The black curves show the attenuation, caused by bubbles, at $50 \mathrm{~Hz}, 400 \mathrm{~Hz}$ and $4 \mathrm{kHz}$. The range of wind speeds typical of a hurricane is also shown. (b) Noise spectral level curves as a function of frequency for typical hurricane wind speeds of 30,50 and $80 \mathrm{~m} / \mathrm{s}$. The black curves show the full areal integration from Eq. (2.5) and the grey curves show the first-order approximation of the field given by Eq. (2.19) with Eqs. (2.15), (2.20) and (2.21). 
of ambient noise level by standard linear least squares estimation, as has been done in Refs. [118] and [38] at low wind speed.

As frequency increases, bubble-layer thickness exceeds a quarter wavelength and the $L_{A}(V(\boldsymbol{\rho}), f)$ term can be approximated as

$$
L_{A}(V(\boldsymbol{\rho}), f) \approx-\alpha(V(\boldsymbol{\rho}), f) L
$$

If we use for illustrative purposes the $L=1.2 \mathrm{~m}$ layer thickness given by Weston,[139] then Eqs. (2.20) and (2.23) agree to within $1 \mathrm{~dB}$ above $300 \mathrm{~Hz}$ and to within $2 \mathrm{~dB}$ between 100 and $300 \mathrm{~Hz}$. While Weston notes that the assumption of a bubble layer

of constant thickness may be poor at high wind speeds, any future improvements in our knowledge of the parameter $L$ can be incorporated in Eqs. (2.18) and (2.23).

The locations of maxima in noise spectral level correspond to the ridge in Fig. 2-6. These can now be approximated analytically by substituting Eqs. (2.15), (2.21) and (2.23) into Eq. (2.19) and taking the derivative with respect to wind speed to obtain

$$
V_{\text {max }} \approx\left\{\begin{array}{cl}
\left(1 /\left(2.15 \times 10^{-7} L \sqrt{f}\right)\right)^{1 / 3} & : \quad 300<f<1.5 k H z \\
\left(1 /\left(5.63 \times 10^{-9} L f\right)\right)^{1 / 3} & : \quad f>1.5 k H z
\end{array}\right.
$$

here assuming $n=3$ and $\alpha(V(\boldsymbol{\rho}), f)$ from Eq. (2.4).

\subsubsection{Accuracy of Underwater Acoustic Wind Speed Esti- mates}

By standard stationary averaging, it should be possible to reduce the variance of an underwater acoustic wind speed estimate enough to be useful for meteorological purposes. Given the relationship $V=H(I)$ between the true wind speed $V$ and true 
ambient noise intensity $I$, the maximum likelihood estimate (MLE) of the wind speed $\hat{V}$ given a measurement of ambient noise intensity $\hat{I}$ is $\hat{V}=H(\hat{I})$ by the invariance of the MLE.[60] The function $H$ can be found either numerically from the exact integration, Eq. (2.5), or analytically from one of the approximations, Eqs. (2.11), (2.19) or (2.22). We define the percent root-mean-square error (RMSE) of the wind speed estimate $\hat{V}$ as

$$
\nu_{r m s e}=100 \frac{\sqrt{\left.<|\hat{V}-V|^{2}\right\rangle}}{<\hat{V}\rangle}
$$

and the percent bias as

$$
\nu_{\text {bias }}=100 \frac{|\langle\hat{V}\rangle-V|}{V}
$$

given

$$
<\hat{V}^{m}>=\int_{0}^{\infty} H^{m}(\hat{I}) p(\hat{I}) d \hat{I}
$$

where $p(\hat{I})$ is the probability density function of the measured intensity $\hat{I}$. For the hurricane noise measurements considered here, where the contributions from a large number of independent sources are received simultaneously, the acoustic field is expected to be a circular complex Gaussian random variable. The time averaged measured intensity $\hat{I}$ is then expected to follow a Gamma distribution[80, 81]

$$
p(\hat{I})=\frac{(\mu / \bar{I})^{\mu} \hat{I}^{\mu-1} \exp \{-\mu(\hat{I} / \bar{I})\}}{\Gamma(\mu)}
$$


where $\mu$ is the time-bandwidth product and $\bar{I}$ is the mean of the noise measurement.

From the full areal integration of Eqs. (2.5) we can numerically find the percent RMSE and percent bias of the wind speed estimate $\hat{V}$. For frequencies below 100 $\mathrm{Hz}$, where attenuation $\alpha$ is insignificant, we find that the percent RMSE and percent bias are functions of $n$ and $\mu$ as shown in Fig. 2-8. At higher frequencies, where attenuation is significant, the percent RMSE and percent bias are also functions of frequency and wind speed. This is illustrated in Fig. 2-9 at a frequency of $400 \mathrm{~Hz}$ assuming $n=3$.

Following the standard practice of stationary averaging, the variance of noise measurements is reduced by inverse the number of stationary samples, $1 / \mu$. In typical ocean acoustic applications, such as matched filtering, $\mu$ 's in excess of 100 are common.[128, 25, 17] For example Piggott[102] and Perrone[96] have obtained measurements of wind noise level with standard deviations less than $1 \mathrm{~dB}$ corresponding[99, $80,81]$ to $\mu>19$.

Given a spectral intensity measurement with $\mu>19$, underwater acoustic wind speed estimates with errors similar to the $6 \%$ to $15 \%$ errors of hurricane-hunting aircraft [41] are possible. For example, at low frequencies where attenuation is insignificant, a measurement of noise spectral level with $\mu=19$ would yield a corresponding percent RMSE in estimated wind speed of 6 to $25 \%$ for the range of published values for $n$ as shown in Fig. 2-8. For the higher frequency $400 \mathrm{~Hz}$ example in Fig. 2-9, where attenuation is significant, a spectral intensity measurement with $\mu=19$ will yield percent RMSEs from 9 to $20 \%$. Even larger errors are common for remote satellite techniques, as high as $40 \%$ as noted in the introduction. From this error analysis we find that underwater acoustic measurements may be worthwhile for estimating hurricane wind speed. Additional errors related to the practical application of the underwater acoustic technique will be discussed in Sec. 2.4.4.

At low frequencies, less than $100 \mathrm{~Hz}$, where attenuation $\alpha$ from bubbles becomes 

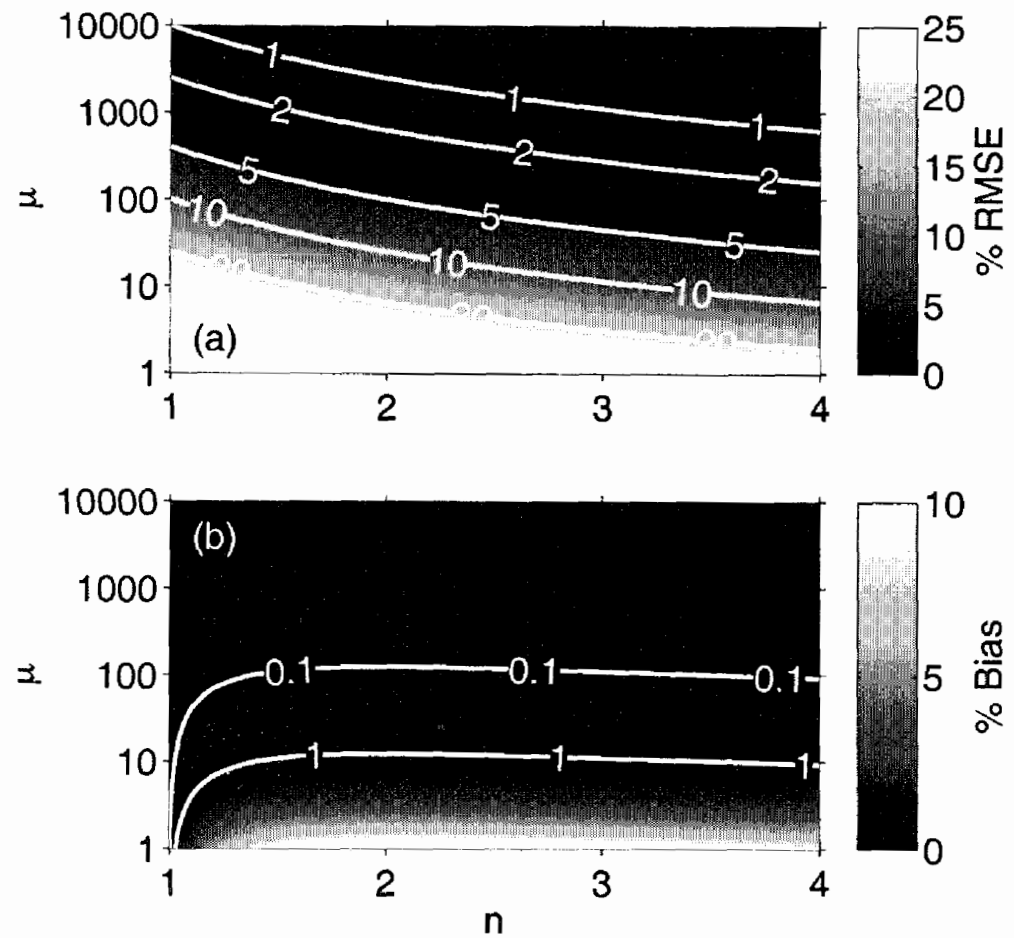

Figure 2-8: The percent RMSE $\nu_{r m s e}$ (a) and percent bias $\nu_{\text {bias }}$ (b) of the wind speed estimate $\hat{V}$ where attenuation by sea-surface bubbles is insignificant, evaluated numerically from Eqs. (2.5) and (2.27). For time-bandwidth products $\mu>5$ the estimate becomes unbiased and the RMSE attains the Cramer-Rao Lower Bound. Piggott[102] and Perrone[96] have measured wind noise level with standard deviations less than $1 \mathrm{~dB}$ which corresponds to $\mu>19$. For $\mu=19$ the percent RMSE in the wind speed estimate ranges from 6 to $25 \%$ depending on $n$ which is a significant improvement over the primary satellite classification method. 

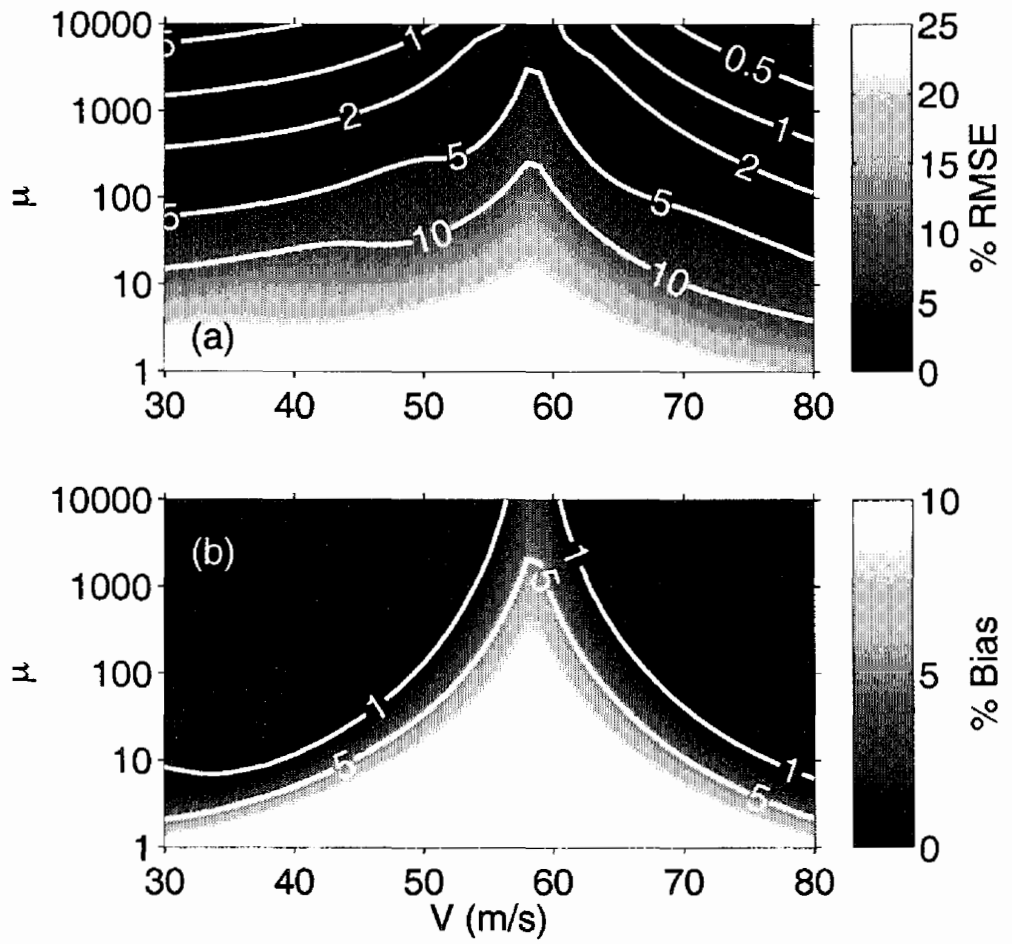

Figure 2-9: The percent RMSE $\nu_{r m s e}$ (a) and percent bias $\nu_{\text {bias }}$ (b) of the wind speed estimate $\hat{V}$ including the effect of attenuation calculated numerically from Eqs. (2.5) and (2.27), assuming $n=3$, at $f=400 \mathrm{~Hz}$ where $V_{\max }=58 \mathrm{~m} / \mathrm{s}$. The error and bias increase for $V \approx V_{\text {max }}$ but for $\mu>5$ and for values of $V$ where $\nu_{b i a s}<1 \%$ the percent RMSE decreases and attains the Cramer-Rao Lower Bound. For spectral intensity measurements with $\mu=19$ the percent RMSE in this example is between 9 and $20 \%$ 
insignificant, the moments of $\hat{V}$ can also be evaluated analytically from the first-order approximation of Eq. (2.22) to illustrate the fundamental parameters affecting a wind speed estimate. The mean of the wind speed estimate can then be written as

$$
<\hat{V}>\approx \frac{\Gamma(\mu+1 / n)}{\Gamma(\mu)}\left(\frac{I(f)}{s_{0} w_{0} \mu}\right)^{1 / n}=\frac{\Gamma(\mu+1 / n)}{\Gamma(\mu) \mu^{1 / n}} V
$$

and the standard deviation as

$$
\sigma_{\hat{V}} \approx\left(\frac{I(f)}{s_{0} w_{0} \mu}\right)^{1 / n} \sqrt{\frac{\Gamma(\mu+2 / n)}{\Gamma(\mu)}-\left(\frac{\Gamma(\mu+1 / n)}{\Gamma(\mu)}\right)^{2}}
$$

At these low frequencies the percent bias can then be approximated as

$$
\nu_{\text {bias }} \approx 100\left|\frac{\Gamma(\mu+1 / n)}{\Gamma(\mu) \mu^{1 / n}}-1\right|
$$

and the percent RMSE as

$$
\nu_{r m s e} \approx 100 \sqrt{\frac{\Gamma(\mu+2 / n) \Gamma(\mu)}{\Gamma(\mu+1 / n)^{2}}-2 \frac{\Gamma(\mu) \mu^{1 / n}}{\Gamma(\mu+1 / n)}+\frac{\Gamma(\mu)^{2} \mu^{2 / n}}{\Gamma\left(\mu+1 / n^{2}\right)}}
$$

These analytic expressions for the percent RMSE and percent bias match those calculated numerically from Eqs. (2.5) and (2.27) and shown in Fig. 2-8 to within $1 \%$.

At low frequencies, where attenuation is insignificant, the Cramer-Rao Lower Bound can be derived from the first-order approximation, Eq. (2.22), as shown in App. B. This provides a straightforward analytic method for calculating the percent 
RMSE as

$$
\nu_{r m s e} \approx 100 \frac{\sqrt{V a r_{\text {asymptotic }}(\hat{V})}}{\langle\hat{V}\rangle}=100 \frac{1}{n \sqrt{\mu}}
$$

which matches the numerically computed value in Fig. $2-8$ for $\mu>5$. This is expected since the Cramer-Rao Lower Bound is the asymptotic variance for large $\mu$. The Cramer-Rao Lower Bound can also be used to calculate the percent RMSE at frequencies above $300 \mathrm{~Hz}$ from the first-order approximation in Eqs. (2.19) with Eqs. (2.15), (2.21) and (2.23) yielding

$$
\begin{aligned}
\nu_{r m s e} & \approx 100 \frac{\sqrt{\operatorname{Var}_{\text {asymptotic }}(\hat{V})}}{\langle\hat{V}\rangle} \\
& =100\left\{\begin{array}{lll}
\frac{1}{\sqrt{\mu}\left(n-6.46 \times 10^{-7} L \sqrt{f} V^{3}\right)} & : f<1.5 k H z \\
\frac{1}{\sqrt{\mu}\left(n-1.69 \times 10^{-8} L f V^{3}\right)} & : f>1.5 k H z
\end{array}\right.
\end{aligned}
$$

which matches the numerical results in Fig. $2-9$ when $\mu>5$ and $\nu_{\text {bias }}<1 \%$.

\subsubsection{Practical Issues}

We have shown that a single underwater acoustic sensor provides significant potential as a measurement tool to accurately estimate local wind speed in a hurricane. There are practical issues, however, to consider when deploying such sensors to monitor a hurricane. While this is not a definitive discussion of all the issues that might be involved, we will attempt to illustrate how an underwater acoustic measurement system might be implemented. For example, how would one deploy these sensors, how many sensors would be needed to fully characterize a hurricane, and how much would it cost. 
One possible scenario would be to deploy multiple sonobouys, similar to those used in weather quantification experiments by Nystuen and Selsor,[90] from aircraft or ships in the path of an oncoming hurricane. As the hurricane passes over each sonobouy the sensor would cut a swath through the storm recording the wind speeds overhead. The swaths from multiple sonobouys could give a fairly complete measurement of the wind speeds in the hurricane. This is similar to the current measurements made by hurricane-hunting aircraft which fly through the storm cutting a swath and measuring wind speed. For both methods, sonobouys or hurricane-hunting aircraft, the sensors must pass through the eye wall of the hurricane where the winds are strongest. For aircraft this means actively piloting the plane through the storm, whereas with stationary sonobouys, one would deploy many sensors along a line that crosses the expected path of the hurricane to insure that at least one sonobouy cuts through the eye wall. For example, a line of 20 sonobouys spaced $5 \mathrm{~km}$ apart across the hurricane's path would span almost $100 \mathrm{~km}$ assuring several measurements of the wind speed in the eye wall. Sonobouys may be ideal for surviving in a hurricane since most of the sensor floats at a depth of several hundred meters and only a small surface expression would be exposed to hurricane winds.

The advantage of deploying sonobouys in advance of a hurricane is that the ship or aircraft never has to enter the storm and would not need to be as expensive as the specialized hurricane-hunting aircraft used today. The cost of a typical hurricanehunting aircraft such as the WC-130 is $\$ 78$ million (inflation adjusted to year 2003 dollars) [2] and the cost of a single flight[9] is roughly $\$ 155,000$. Between two and eight aircraft flights are made per day[41] for potentially landfalling hurricanes in the North Atlantic where the lifespan of a hurricane can be several weeks. Twenty sonobouys, at $\$ 500$ each,[85] could be deployed from inexpensive non-specialized ships or aircraft in the path of an oncoming hurricane well before conditions are dangerous for roughly $\$ 10,000$. 
An alternative scenario would be to deploy hundreds of permanent shore-cabled hydrophone systems, at $\$ 10,000$ to $\$ 20,000$ each depending on cable length, in strategic hurricane prone areas for a few million dollars. As noted before, this is much less than the purchase price of a WC-130 hurricane-hunting aircraft.

Such underwater acoustic systems would likely be used in conjunction with apriori location estimates from satellites. Satellites would determine the path of the hurricane relative to the hydrophone and show whether the sensor passed through the high winds of the eye wall. The underwater acoustic measurement would then provide an estimate of the wind speeds for the portions of the hurricane that passed overhead. If a hydrophone does not pass through the powerful eye wall but rather through the weaker surrounding winds it would still provide a lower bound or threshold measurement of wind speed and it may be possible to extrapolate these lower wind speeds to determine the higher wind speeds of the eye wall.

\subsection{Hydrophone Array Analysis}

The analysis in the previous sections demonstrates how omnidirectional sensors may be used to accurately measure the local winds and quantify the destructive power of a hurricane as it passes overhead. It may be possible to use arrays of hydrophones to beamform on the acoustic field from a hurricane at long range. For illustrative purposes we will consider horizontal linear arrays of the type that might be towed from an oceanographic or naval vessel, however, other array configurations, such as moored arrays, might also be useful. Arrays might also be useful for directionally filtering out other noise sources, such as ships and surf, in local measurements.

Using the expression for the cross-spectral density of the noise field of Eq. (2.2) we find the angular spectral density of the noise received by an $N$ element array, or beamformed output, to be 


$$
B(\Omega, f)=\frac{1}{N^{2}} \sum_{m=1}^{N} \sum_{n=1}^{N} e^{-j \mathbf{k} \cdot \mathbf{r}_{\mathbf{m}}} C\left(\mathbf{r}_{\mathbf{m}}, \mathbf{r}_{\mathbf{n}}, f\right) e^{j \mathbf{k} \cdot \mathbf{r}_{\mathbf{n}}}
$$

with units of $\mu \mathrm{Pa}^{2} / \mathrm{sr}^{2} \mathrm{~Hz}$, where $\mathbf{k}=\frac{2 \pi f}{c} \mathbf{i}_{\mathbf{\Omega}}, \mathbf{i}_{\mathbf{\Omega}}$ is a unit vector in the steering direction $\Omega$, and $\mathbf{r}_{\mathbf{m}}$ is the position of the $m^{\text {th }}$ hydrophone on the array.

We define the hurricane wind-generated noise source area to include sources within $200 \mathrm{~km}$ of the hurricane's center as shown in Fig. 2-1 and the ambient noise source area to include sources generated by the $5 \mathrm{~m} / \mathrm{s}$ winds surrounding the hurricane. To show how an array might be able to measure the destructive power of a hurricane, the angular spectral density of the noise will be calculated for a hurricane as a function of maximum wind speed.

The angular spectral density of Eq. (2.35) in the direction of the hurricane increases with maximum wind speed, as shown in Fig. 2-10 for an array at 200-m depth at a range far from the hurricane eye. The difference in spectral density between the strong $72-\mathrm{m} / \mathrm{s}$-wind-speed and weak $33-\mathrm{m} / \mathrm{s}$-wind-speed hurricanes of Fig. $2-10$ is roughly $10 \mathrm{~dB}$ given the assumption here that $n=3$. The difference in spectral density would be greater for larger $n$.

A practical horizontal array can resolve the important features of the hurricane such as the eye wall, which has dimensions of tens of kilometers, when placed in a convergence zone as in Figs. 2-10(a) and (c). This is not possible for an array just outside the convergence zone as shown in Fig. 2-10(b). In the former case, the length $L$ of an array oriented at broadside to the hurricane, would have to be

$$
L>R \lambda / l
$$

where $R$ is the range from the array to the hurricane and $l$ is the size of the eyewall. Typical linear arrays[128] have lengths $L$ on the order of $100 \frac{\lambda}{2}$. In the example of Fig. 2-10, a broadside array with $L=32 \lambda$, similar to the ONR FORA array,[1] 

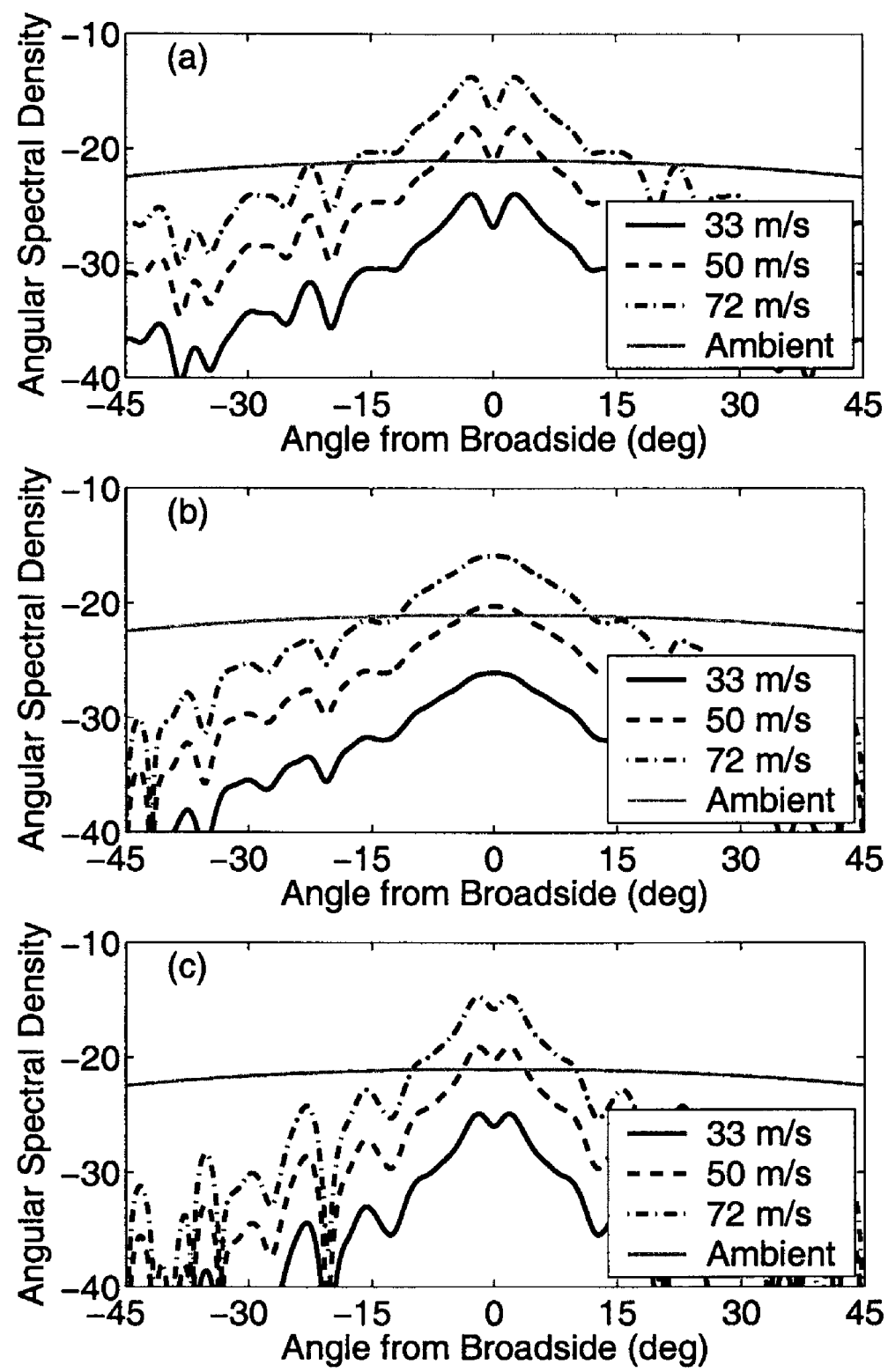

Figure 2-10: Angular spectral density level $10 \log (B(\Omega, f))\left(\mathrm{dB}\right.$ re $\left.\frac{\rho_{\mathrm{w}} \mathrm{CI} \mathrm{I}_{\mathrm{ref}}}{\mathrm{sr}^{2}}\right)$ at $100 \mathrm{~Hz}$ for a 64 -element $\lambda / 2$-spaced horizontal broadside array as a function of steering angle for hurricane generated noise in the North Atlantic at ranges of $257 \mathrm{~km} \mathrm{(a),} 289 \mathrm{~km}$ (b), and $385 \mathrm{~km}$ (c) from the eye of the hurricane, assuming $n=3$. Ranges of 257 and $385 \mathrm{~km}$ correspond to the fourth and sixth convergence zones from the center of the hurricane. $289 \mathrm{~km}$ is exactly between the fourth and fifth convergence zones. Curves are shown for a powerful $72 \mathrm{~m} / \mathrm{s}$ hurricane, a medium $50 \mathrm{~m} / \mathrm{s}$ hurricane and a weak $33 \mathrm{~m} / \mathrm{s}$ hurricane. The angular spectral density level from ambient noise is plotted for comparison. A steering angle of $0^{\circ}$ corresponds to the array steered toward the calm eye of the hurricane and the powerful eye wall is located at $\pm 3^{\circ}$. This array has an angular resolution of $1.8^{\circ}$ which at a range of $257 \mathrm{~km}$ corresponds to a $8 \mathrm{~km}$ spatial resolution. 
images the hurricane with $10 \mathrm{~km}$ resolution at a range of $320 \mathrm{~km}$. The width of the convergence zone must also be sufficiently small in range to resolve the eye wall. For the given environment and ranges considered, this condition is satisfied because the convergence zone width in range is roughly $5 \mathrm{~km}$, which is less than the width of the eye wall.

A horizontal array oriented at end-fire to the hurricane has the advantage that it discriminates against local surface noise coming from near broadside in favor of sound that travels from long distances at shallow angles in the waveguide. This could potentially lead to longer hurricane detection ranges. Unfortunately, at end-fire, the length of the array must satisfy

$$
L>2 \lambda(R / l)^{2}
$$

to resolve the eye-wall. For example an impractically long $L=2000 \lambda$ end-fire array would be needed to achieve $10 \mathrm{~km}$ resolution at a range of $320 \mathrm{~km}$.

The analysis presented here for the North Atlantic shows that it may be possible to image the features of a hurricane using linear broadside arrays of sufficient length. Waveguides that lack excess depth, such as the Bay of Bengal, do not exhibit the convergence zone structure seen in the North Atlantic. This probably makes it extremely difficult to even detect hurricanes using practical linear arrays at long ranges in these environments.

\subsection{Conclusions}

We have shown that the wind-generated noise received by a single underwater acoustic sensor in a hurricane can be well approximated by sea-surface contributions so local that wind speed and surface source intensity can be taken as nearly constant. Two terms with empirically and analytically determined dependencies may be used 
to estimate wind speed from measured ambient noise spectral level (1) a universal ambient noise source term and (2) a local waveguide calibration term. At low frequencies, current evidence suggests a simple power-law relationship exists between noise intensity and wind speed so that the log of wind speed may be estimated accurately from spectral ambient noise level by linear least square estimation. At higher frequencies, a non-linear relationship is expected but we show that it should be possible with broadband measurements to make unambiguous low-variance wind speed estimates from broad-band noise measurements. 


\section{Chapter 3}

\section{Quantifying Wind Speed in \\ Hurricane Gert with Undersea}

\section{Sound}

\subsection{Introduction}

In this chapter we demonstrate, based on experimental measurements, that inexpensive ocean acoustic sensors may be used to accurately quantify the destructive power of a hurricane by measuring the wind speeds to within $5 \%$ accuracy. This is similar to the accuracy achieved by hurricane-hunting aircraft.[41] To do this we correlate the undersea noise generated by hurricane Gert,[71] recorded by an autonomous acoustic sensor, [37] with meteorological data from reconnaissance aircraft and satellites. From this correlation we find that the intensity of low frequency sound generated by the hurricane is approximately proportional to the cube of the local wind speed.

Accurate hurricane quantification is critical for disaster planning in order to mitigate the destructive effect of these storms. The destructive power of a tropical cyclone was recently demonstrated by hurricane Katrina which caused over 1000 fatalities[29] 
and an estimated economic loss of 100 billion dollars[10]. Prior to Katrina, the United States Commission on Ocean Policy emphasized the need for more accurate quantifications of hurricane destructive power to improve disaster planning.[133]

As discussed in Chapter 1, the standard method for satellite hurricane classification is the Dvorak method [31] where hurricane cloud features observed in satellite images are interpreted to estimate wind speed and classify destructive power. This method can yield errors in wind speed estimates exceeding $40 \%[93,43,12,4,121]$ (when compared to the best-estimate wind speed from aircraft measurements). For example, of the eight North Atlantic hurricanes of 2000, three of them [93, 43, 12] experienced Dvorak errors over $40 \%$ and three more $[94,72,120]$ experienced Dvorak errors over $20 \%$. Several satellite microwave techniques show some promise for measuring hurricane wind speed [59] but because of resolution and accuracy issues the Dvorak method is still the standard for satellite hurricane classification [44]. More accurate classification can be achieved by flying specialized 'hurricane-hunting' aircraft through the high winds of a hurricane, but their expense prohibits their routine use outside of the United States [33]. For example, the cost to purchase a WC-130 aircraft is roughly $\$ 82$ million [2] (adjusted for inflation to year 2005 dollars) and the deployment cost is $\$ 155,000$ per flight [9]. Given these limitations in current capability, the United States Commission on Ocean Policy has recommended that future ocean observing systems be used to improve weather related warnings [133]. We experimentally demonstrate that underwater acoustic measurements of noise intensity may provide a useful technique for hurricane wind speed estimation and quantification.

The physical mechanisms for ocean noise generation by wind are not entirely understood. The entrainment of oscillating bubbles by wind-generated waves is believed to be the dominant natural source mechanism at frequencies between $10 \mathrm{~Hz}$ and 10 $\mathrm{kHz}$ with a broad peak between 300 and $600 \mathrm{~Hz} .[67,136,102,23]$ The non-linear interactions of ocean waves has been proposed as the dominant mechanism between 
0.1 and $10 \mathrm{~Hz}$ with a peak at roughly $0.3 \mathrm{~Hz} \cdot[134,22]$ In the 10 to $50 \mathrm{~Hz}$ frequency range considered here, it is likely that the oscillation of wave-entrained bubbles is the dominant effect. Regardless of the specific physical mechanism, the relationship between noise intensity and wind speed has been extensively studied experimentally at low non-hurricane wind speeds, $[118,38]$ but never previously at hurricane wind speed to our knowledge due to the extraordinary difficulty of conducting experiments at sea in hurricane conditions.

The entrainment of bubbles may play a role in the attenuation of sound in the ocean, particularly at high frequencies and wind speeds.[40] At the low frequencies and hurricane wind speeds considered here, attenuation by entrained bubbles is expected[141] and found to be negligible.

\subsection{Methods}

In 1999 the United States National Oceanographic and Atmospheric Administration's (NOAA) Pacific Marine Environmental Laboratory (PMEL) moored an undersea acoustic sensor at a depth of roughly $800 \mathrm{~m}$ in the $4.7 \mathrm{~km}$ deep North Atlantic at $17.7^{\circ} \mathrm{N} 49.5^{\circ} \mathrm{W}$ with the purpose of recording seismic events along the mid-Atlantic ridge.[37] On September 15th, 1999 this hydrophone recorded the underwater sound generated by hurricane Gert[71] as it passed overhead (Fig. 3-1).

The measured acoustic intensity in the 10 to $50 \mathrm{~Hz}$ band, shown in Fig. 3-2, changed with time first reaching a maximum, then a minimum, and then another maximum. This variation is consistent with the advection of the characteristic geometry of the hurricane over the hydrophone with the first maximum in the sound intensity corresponding to the leading-edge eye wall winds, the minimum by the low wind speeds of the eye, and the later maximum by the trailing edge of the eye wall.

In order to reduce the uncertainty of these noise measurements, we average the 


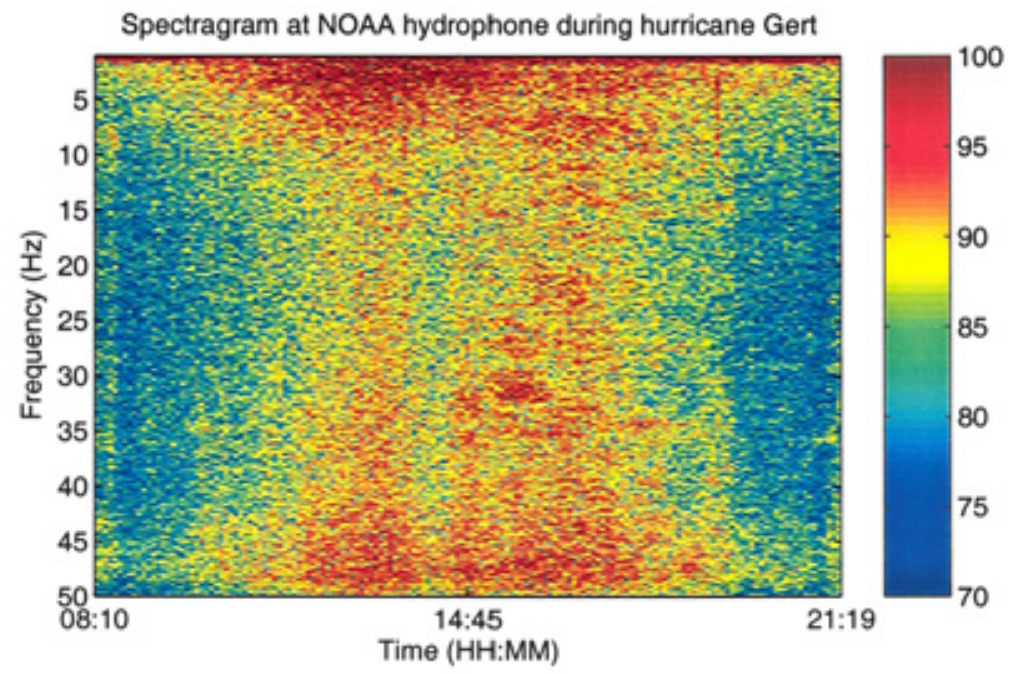

Figure 3-1: Spectrogram of the hurricane noise in $\mathrm{dB}$ re $\mu \mathrm{Pa}^{2} / \mathrm{Hz}$, from 1 to $50 \mathrm{~Hz}$, received by the NOAA hydrophone on 15 Sept. Above $10 \mathrm{~Hz}$ the maximum between 13:00 and 16:00 correspond to the powerful winds of the hurricane's eye-wall. At $1 \mathrm{~Hz}$ we see noise most likely due to cable strum. There seems to be some hurricane related noise between 1 and $10 \mathrm{~Hz}$, however it does not correlate well with wind speed and may be caused by nonlinear surface wave interaction as discussed in the next chapter. 


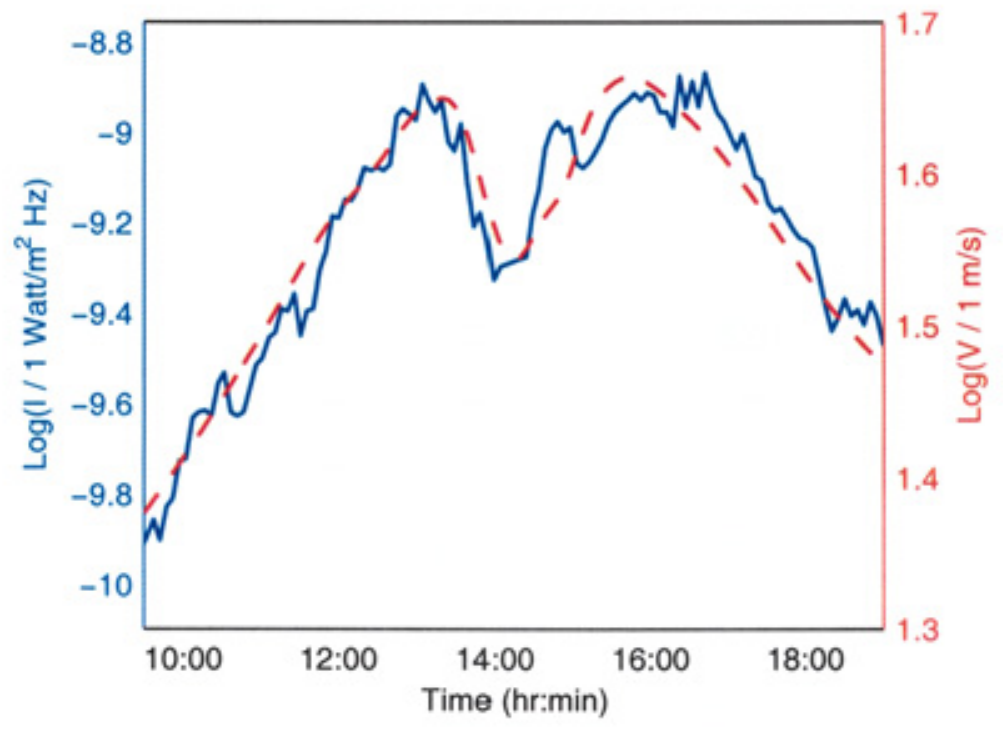

Figure 3-2: $\log$ of the noise spectral intensity $I$ (solid blue) in Watts $/ \mathrm{m}^{2} \mathrm{~Hz}$, from 10 to $50 \mathrm{~Hz}$, received by the NOAA hydrophone on 15 Sept. The expected intensity (red dashed line) is based on the hindcast wind speed $V$ in $\mathrm{m} / \mathrm{s}$ above the hydrophone given the best fit relation $\log \left[I /\left(1 \mathrm{Watt} / \mathrm{m}^{2} \mathrm{~Hz}\right)\right]=n \log [V /(1 \mathrm{~m} / \mathrm{s})]+b$ where $n=3.36$ and $b=-14.46$. The maxima at 13:30 and 15:30 correspond to the powerful winds of the hurricane's eye-wall and the minimum at 14:30 corresponds to the hurricane's eye. The percent root-mean-square error between the two curves is $5 \%$. 
received intensity over the 10 to $50 \mathrm{~Hz}$ band and over 10 minute consecutive time windows. This leads to a time-bandwidth product $\mu=24000$ which can be thought of as the number of independent samples in the average. By the method of stationary averaging[80] the standard deviation $\sigma_{L}$ of the $\log L=\log \left(I /\right.$ Watt $\left./ \mathrm{m}^{2} \mathrm{~Hz}\right)$ of the averaged intensity $I$ can be given as $\sigma_{L}=\log (e) \sqrt{1 / \mu}$ for $\mu>>1$. For the $\log$ of the averaged intensities shown in Fig. 1, the standard deviation is then $\sigma_{L}=0.003 \mathrm{~dB}$ re Watt $/ \mathrm{m}^{2} \mathrm{~Hz}$.

No direct measurements of wind speed were taken at the sensor's location so we synthesize the local wind speed record based on available data from United States Air Force (USAF) hurricane-hunting aircraft and NOAA hurricane tracking satellites. We begin by estimating the hurricane's track to determine its location relative to the acoustic sensor over time. NOAA satellites gave location estimates of Gert every six hours with error margins[115] of $\pm 28 \mathrm{~km}$ and USAF aircraft gave location estimates[30] with error margins[41] of $\pm 11 \mathrm{~km}$. These two sets of location estimates yield two different possible tracks hurricane Gert could have taken past the acoustic sensor. Based on satellite data hurricane Gert passed $11 \mathrm{~km}$ to the South of the acoustic sensor at 14:15 on 15 Sept., however, based on aircraft data the storm passed $32 \mathrm{~km}$ to the South of the acoustic sensor at 13:49. To resolve this ambiguity we consider an ensemble of possible tracks hurricane Gert could have taken past the sensor spanning the error margins in the measurements, with distances from $50 \mathrm{~km}$ to the South to $20 \mathrm{~km}$ to the North of the sensor and times from 12:00 to 16:00. Also, we consider a range of typical values for hurricane translation speed, from 5 to 15 $\mathrm{m} / \mathrm{s}$.

In addition to providing hurricane location estimates, USAF hurricane-hunting aircraft were used to measure the wind speed structure in the hurricane,[30] normalized[104] to an altitude of $10 \mathrm{~m}$. The measurements taken on 16 Sept., a day after the hurricane passed the acoustic sensor and the first day aircraft measurements were made, show 
a wind speed structure typical of a hurricane with a low wind speed eye surrounded by the high $47 \mathrm{~m} / \mathrm{s}$ wind speeds of the eye wall (Fig. 3-3). To reconstruct the wind speed record at the undersea sensor location as Gert passed overhead, we advect this wind speed structure backward in time in the directions determined by the ensemble of possible hurricane tracks. Even though the aircraft wind speed measurement was taken nearly a day after the acoustic measurement, it should be possible to hindcast

the wind speed record since evidence indicates that the hurricane wind speeds remained roughly constant during that time. This can be seen from satellite estimates, which indicate a less than $10 \%$ increase in maximum hurricane wind speed[71], and from NOAA meteorological forecast models, which predict no change in maximum wind speed[115], over the 24-hour period.

For each possible hurricane track the power-law hypothesis is tested by calculating the linear regression between the $\log$ of the intensity $I$ and the log of the local wind speed $V$ (Eq. (3.2)). The best-fit hurricane track is taken as the one where the rootmean-square error (RMSE) of the linear regression is minimized. For this best-fit track, hurricane Gert passed $29 \mathrm{~km}$ to the South of the NOAA undersea acoustic sensor at 14:15 moving at a speed of about $12 \mathrm{~m} / \mathrm{s}$. This is within the error margins of the aircraft and satellite location estimates. Given this best-fit track the log of the local wind speed above the sensor may be plotted over time (Fig. 1).

\subsection{Results}

From this regression analysis, wind speed $V$ and undersea noise intensity $I$, which is a scalar magnitude proportional to the magnitude square of the complex acoustic pressure, follow a simple power law relationship

$$
I(V)=V^{n} W
$$




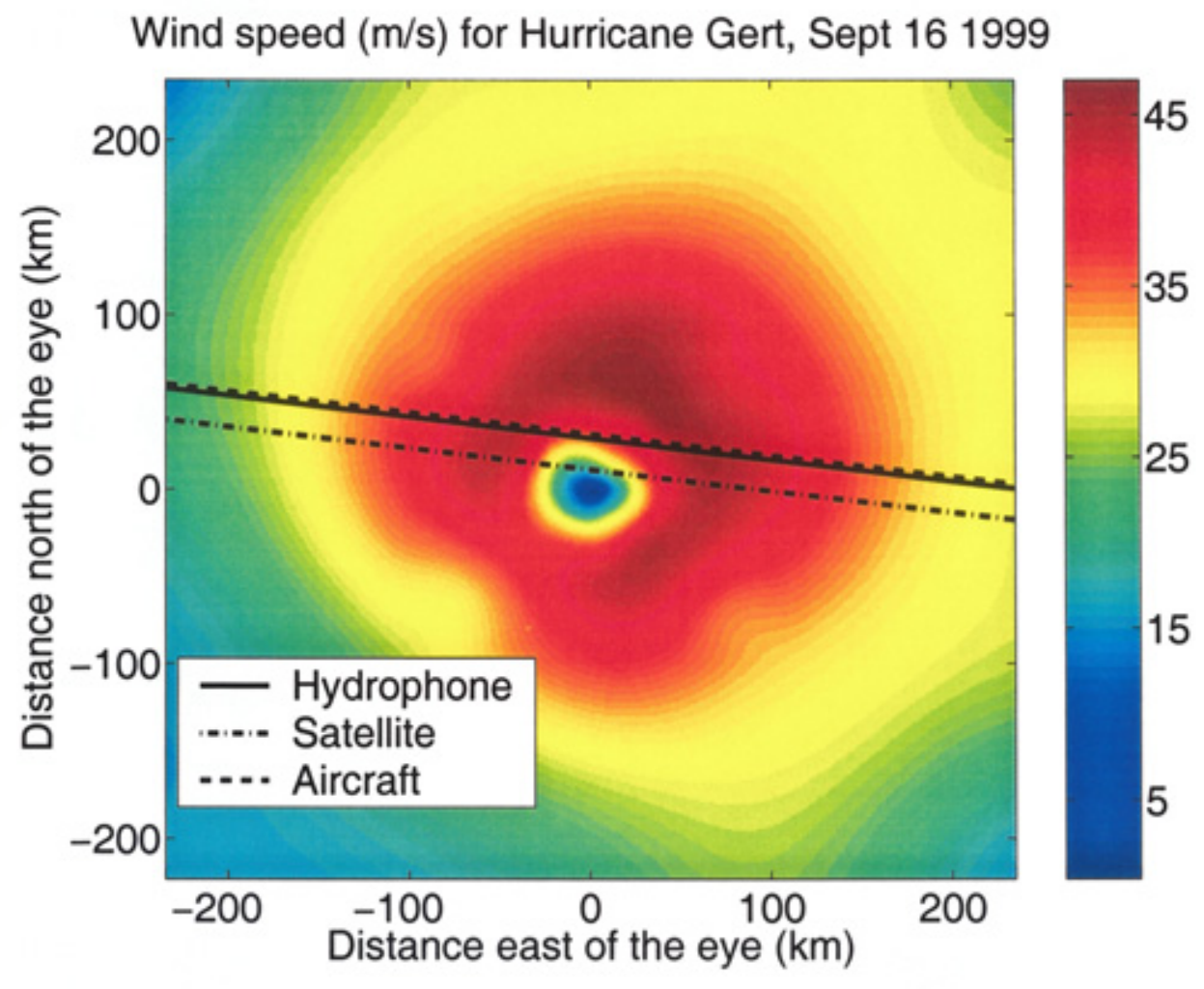

Figure 3-3: Wind speed in $\mathrm{m} / \mathrm{s}$ in hurricane Gert as a function of position relative to the center or 'eye'. As hurricane Gert passed over the acoustic sensor the sensor effectively sliced a path through the hurricane. The lines show the paths estimated based on Satellite (dash-dot), Aircraft (dashed) and Acoustic best fit (solid) respectively. 
as shown in Fig. 3-4, where $W$ is a waveguide propagation factor.[141] Taking the logarithm this becomes a linear relationship

$$
\log \left(\frac{I(V)}{1 \mathrm{Watt} / \mathrm{m}^{2} \mathrm{~Hz}}\right)=n \log \left(\frac{V}{1 \mathrm{~m} / \mathrm{s}}\right)+b
$$

where $n=3.36$ and $b=-14.46$ in the low frequency band of the acoustic measurements (Fig. 2). The slope $n$ is universal and independent of measurement position, while the intercept $b$ is a calibration that depends on local waveguide environment.[141]

This correlation between undersea noise and local wind speed demonstrates that acoustic sensors can be used as anemometers for estimating hurricane wind speed. This is illustrated in Fig. 1 where the hurricane wind speed closely follows the log of the noise intensity. Additionally, an acoustic sensor deployed to pass under a hurricane's eye wall could estimate the storms total destructive power because hurricane power is proportional to the cube of the maximum wind speed.[53]

The log of wind speed can be then found from measurements of ambient noise level by standard linear least squares estimation, as has been done by Shaw, Watts and Rossby[118] and Evans, Watts, Halpern and Bourassa[38] for low $(<10 \mathrm{~m} / \mathrm{s})$ spatially-uniform wind speeds. The empirical fit in Fig. 2 shows that wind speed can be determined from undersea noise intensity to within a fractional error of 0.05 , or $5 \%$, where fractional error is defined as

$$
\nu=\frac{\sqrt{\left.<|\hat{V}-V|^{2}\right\rangle}}{V}
$$

and where $\hat{V}$ is the estimated wind speed from undersea acoustic measurements and $V$ is the actual wind speed. This is similar to the $5 \mathrm{~m} / \mathrm{s}$ errors typical of aircraft estimates.[41] It should be noted that satellite estimates[116] of Gert's wind speed 


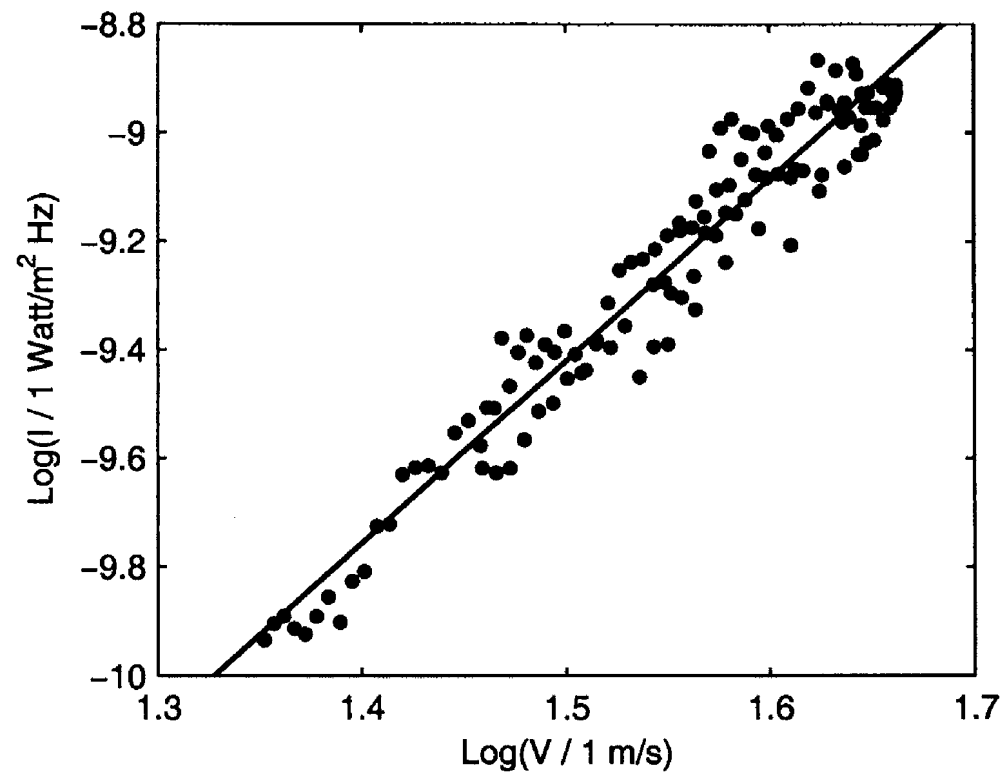

Figure 3-4: The $\log$ of the noise spectral intensity $I$ in Watts $/ \mathrm{m}^{2} \mathrm{~Hz}$ versus the $\log$ of the surface wind speed $V$ in $\mathrm{m} / \mathrm{s}$ at the NOAA hydrophone (circles) based on the best fit hurricane track. The best fit linear regression shows a $\log \left[I /\left(1 \mathrm{Watt} / \mathrm{m}^{2} \mathrm{~Hz}\right)\right]=$ $3.36 \log [V /(1 \mathrm{~m} / \mathrm{s})]-14.46$ relationship between intensity and wind speed with a percent root-mean-square error of only $5 \%$. 
were much less accurate and overestimated the wind speed by roughly $40 \%$ when compared with aircraft estimates[30] made near the time of the acoustic measurement.

The results of Figs. 3-2 and 3-4 are based on the 'best fit' track which is the hurricane track for which the RMSE of the linear regression is smallest. The other possible tracks that hurricane Gert could have taken must also be considered. Figure 3-5 shows the RMSE and slope $n$ of the linear regression for a range of other possible hurricane tracks. While the minimum RMSE of $0.76 \mathrm{~dB}$ corresponds to a slope $n=3.36$, there is a range of tracks with small RMSEs, less than $0.8 \mathrm{~dB}$, which correspond to slopes $n$ between 3 and 3.6. We see then that, due to the uncertainty in our knowledge of wind speed at the hydrophone location, there is some uncertainty in the slope of the relationship between log of wind speed and log of acoustic intensity.

This uncertainty can be somewhat alleviated be comparing the data from hurricane Gert with data gathered by other researchers at low non-hurricane wind speeds. Figure 3-6 shows the linear regression between the $\log$ of intensity and $\log$ of wind speed including low-wind-speed data gathered by Cato and Tavener [23]. With this additional data the slope of the regression becomes $n=3.34$ which is effectively the same as that given in Fig. 3-4.

It should be noted that in Fig. 3-6 the variance or spread in the data from Cato and Tavener [23] is significantly greater than in the data from hurricane Gert. There are three factors that may contribute to this increased variance. First, the time-bandwidth product for the data samples from Gert is significantly greater, by a factor of more than 100, than for the data from Cato and Tavener. This results in greater stationary averaging of the data from Gert. Second, the acoustic sensor used for Gert was at a depth of $800 \mathrm{~m}$ compared to $40 \mathrm{~m}$ for the measurements of Cato and Tavener. This leads to increased spatial averaging over more surface noise sources in the hurricane data. Last, the low-wind-speed measurements may be corrupted by other non-wind-related noise sources. These three effects may contribute to the 


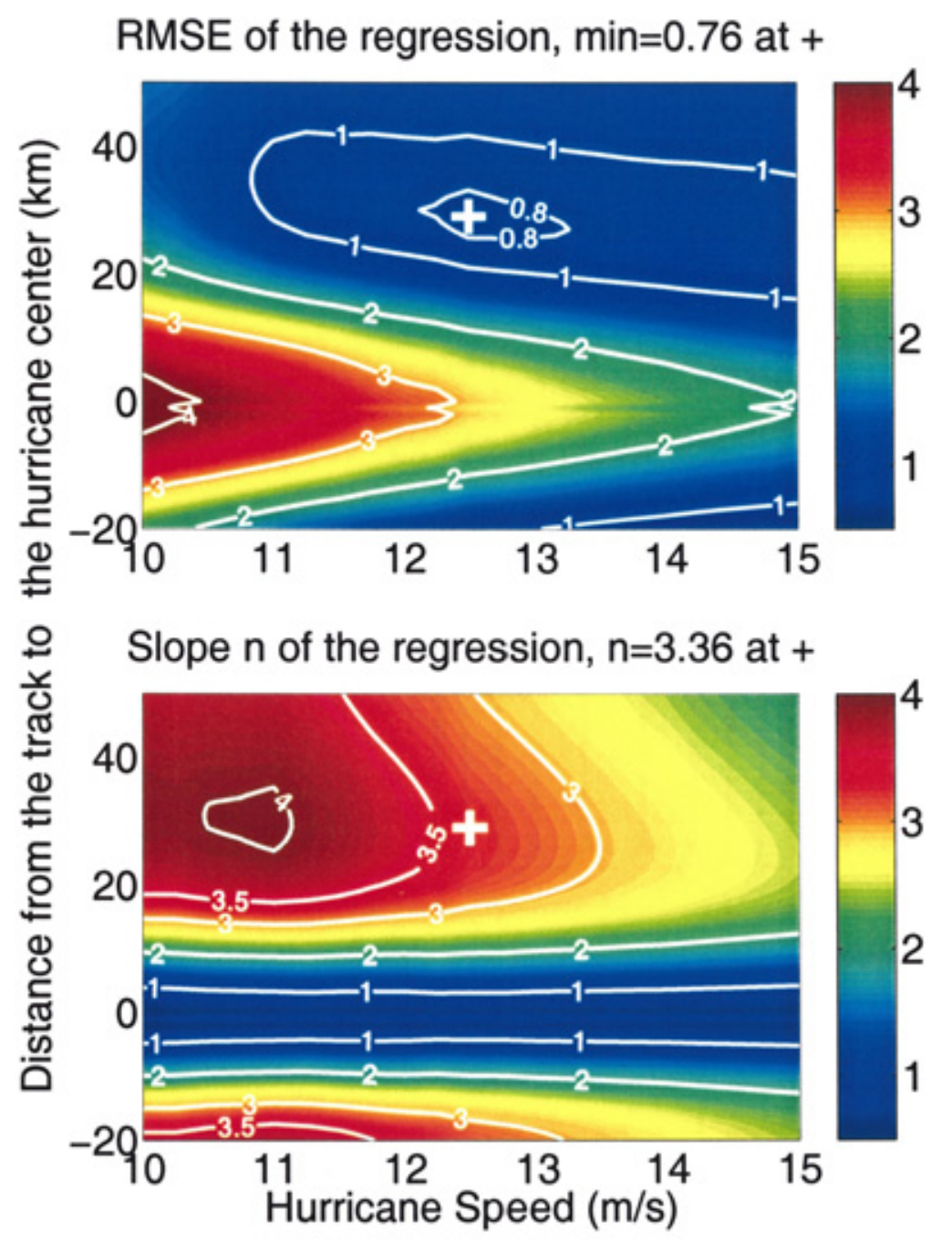

Figure 3-5: The upper plot gives the root-mean-square error (RMSE) of the linear regression for each possible hurricane track. The $y$-axis is the north-south distance between the hydrophone and the possible hurricane track and the $\mathrm{x}$-axis is the speed at which the hurricane was moving. The minimum RMSE of $0.76 \mathrm{~dB}$, indicated $\mathrm{y}$ the + symbol, is taken to represent the 'best fit' hurricane track. Note that the RMSE is small, less than $0.8 \mathrm{~dB}$, over a range of possible tracks. The lower plot gives the slope $n$ of the linear regression for each possible hurricane track with the same $\mathrm{x}$ and y axes as the upper plot. For the best track the slope $n=3.36$. For the range of tracks with a RMSE less than $0.8 \mathrm{~dB}$, the slope of the regression could vary from 3 to 3.6 . 


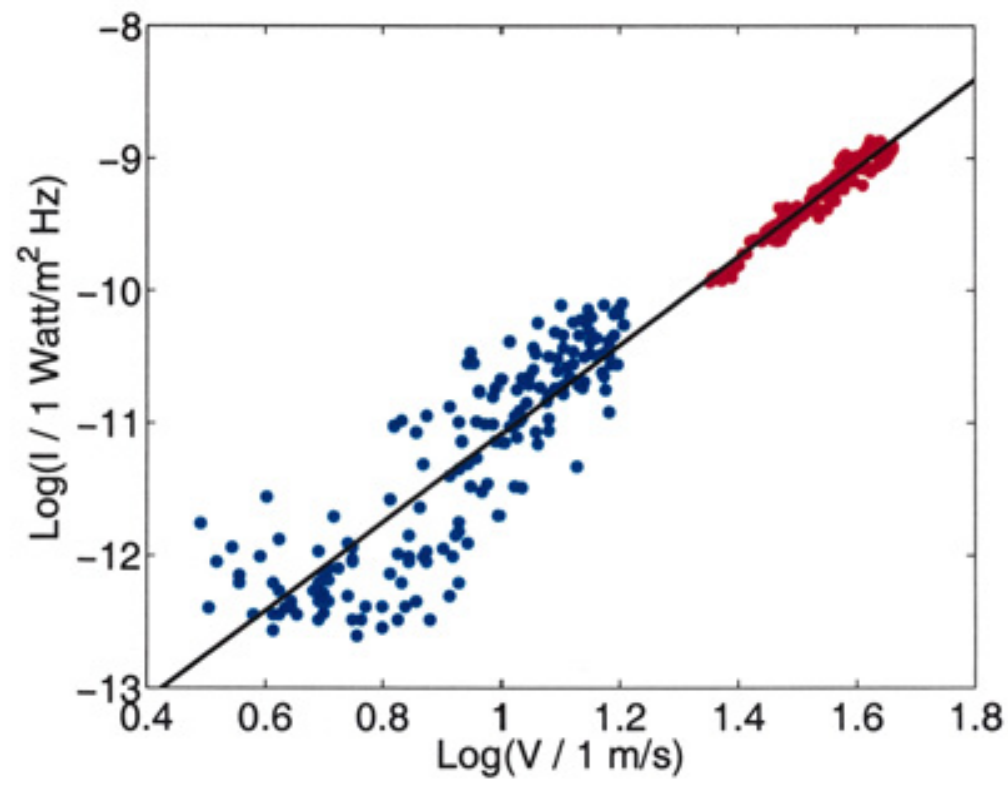

Figure 3-6: $\log$ of noise intensity $I$ versus $\log$ of hindcast local wind speed $V$ based on the best-fit track (red circles). Also plotted is the $\log$ of noise intensity $I$ versus $\log$ of local wind speed $V$ measured by Cato and Tavener [23] (blue circles) at lower non-hurricane wind speeds off Australia. The linear regression shows a $\log \left(I / 1 \mathrm{Watt} / \mathrm{m}^{2} \mathrm{~Hz}\right)=3.34 \log (V / 1 \mathrm{~m} / \mathrm{s})-14.5$ relationship between noise intensity $I$ and wind speed $V$. 
increase accuracy of the acoustic measurements in hurricane Gert.

\subsection{Conclusions}

Our theoretical calculations[141] show that an omnidirectional sensor at the depth of the NOAA sensor $(800 \mathrm{~m})$ in a deep-water environment like the North Atlantic effectively measures the noise generated by sea-surface winds within a horizontal radius $(\approx 2 \mathrm{~km})$ that is much smaller than the typical length scales of a hurricane making it ideal for hurricane wind speed measurements. A number of distributed point sensors, similar to those used in weather quantification experiments by Nystuen and Selsor,[90] could be scattered from aircraft or ships in the path of an oncoming hurricane. Each sensor would then cut a swath through the storm recording the wind speeds overhead. This is analogous to the procedure followed by hurricanehunting aircraft which fly an 'X-pattern' through a hurricane cutting two swaths while measuring wind speed.[41]

The advantage of deploying sonobouys in advance of a hurricane is that the ship or aircraft never has to enter the storm and would not need to be as expensive as the specialized hurricane-hunting aircraft used today. Several sonobouys, at $\$ 500$ each (M. May, personal communication), could be deployed from inexpensive non-specialized ships or aircraft in the path of an oncoming hurricane well before conditions are dangerous. While it is unlikely that undersea acoustic techniques would render aircraft obsolete, this technique would provide inexpensive hurricane monitoring capability for areas of the world not currently covered by specialized hurricane-hunting aircraft such as the Pacific and Indian Oceans. In the North Atlantic where specialized aircraft are already in use, acoustic techniques may make it possible to reduce the number of aircraft flights necessary.

Based on measurements of undersea noise from hurricane Gert, we demonstrate 
a new method for estimating hurricane wind speed and power. The power-law relationship and high correlation between noise intensity and local hurricane wind speed show that estimates of hurricane wind speed based on undersea noise measurements could be very accurate with an error margin of only $5 \%$. An undersea acoustic sensor could then effectively act as an acoustic anemometer, providing an accurate estimate of a hurricane's wind speeds and destructive power. Currently many ocean acoustic systems exist that could be used for meteorological measurements, such as the NOAA sensor used in this work, and additional systems could be deployed from ships, aircraft, or near shore at relatively low cost. 


\section{Chapter 4}

\section{Modeling Microseism Generation}

\section{by Inhomogeneous Ocean Surface}

\section{Waves in Hurricane Bonnie Using}

\section{the Non-linear Wave Equation}

\subsection{Introduction}

Hurricanes generate seismic noise, commonly referred to as microseisms, in the 0.1 to $0.6 \mathrm{~Hz}$ frequency range. Here we describe the microseisms generated by the spatially inhomogeneous waves in a hurricane. Using the ocean surface directional wave spectrum in hurricane Bonnie $[142,88]$, we hindcast the microseismic field and compare it with seismic measurements from Florida. Previously hurricane surface directional wave spectra had not been adequately measured or modeled so that researchers had to rely on assumed spectra in order to model hurricane microseisms [78]. This analysis is useful because microseisms are a primary cause of noise in seismic measurements $[74,107,105]$ that raise the detection threshold for monitoring earthquakes [135] 
and tsunamis. Historically microseismic measurements have also been used to track hurricanes $[107,45]$ although that task is now accomplished using satellites.

We present an analytic expression for microseism generation by spatially inhomogeneous waves typical in a hurricane based on the non-linear wave equation where a second-order seismo-acoustic field is generated by a source distribution which depends on the first-order ocean surface wave motion. The seismo-acoustic field at a receiver can then be expressed as the integral over the source distribution multiplied by the waveguide Green function. This expression is ideal for hurricane generated microseisms since it can be used to calculate the acoustic field due to spatially inhomogeneous surface waves. Also, this expression may be used in range-dependent waveguide environments as is the case when a hurricane at sea generates microseisms that propagate up the continental margin to a receiver on land.

In the past, microseism generation has been modeled for idealized hurricanes [78] where the ocean surface wave field within the hurricane was taken to be spatially homogeneous and the ocean waveguide to be range-independent. Previous microseismic models, however, cannot be applied in typical hurricane scenarios because the surface wave fields are inhomogeneous in that the wave height spectra in different parts of the hurricane can vary both in magnitude and directionality. In some cases this is because spatial homogeneity is assumed over infinite $[51,16,54,77,62]$ or very large $[78,63,64]$ surface wave areas. One model that does account for finite microseismic source generation regions is limited by the assumption that the receiver is at the center of the source area [21, 22]. These model requirements are summarized in Table 4.1. Also, these previous models are not applicable to the range-dependent environments typical in hurricane measurements where microseisms generated by a hurricane over the deep ocean are measured by a sensor on land. Our non-linear wave equation method is shown to agree with earlier models $[54,77]$ if we make the same simplifying assumptions that the source generation region is spatially homogeneous 
Table 4.1: Previous microseism models and their assumptions

\begin{tabular}{|c|c|c|c|c|c|c|}
\hline Author & 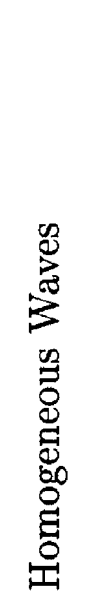 & 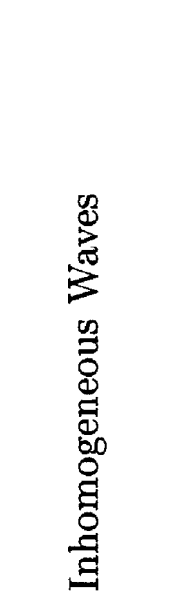 & 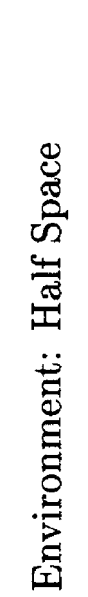 & 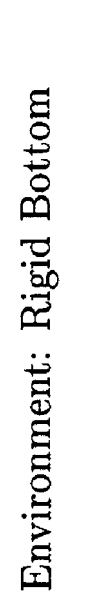 & 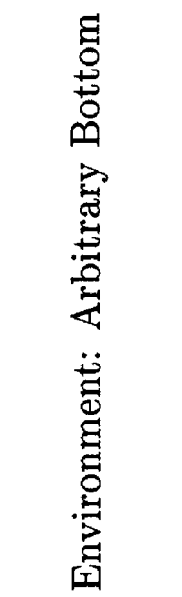 & 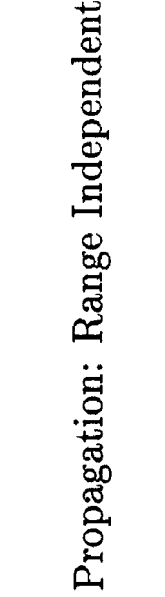 \\
\hline Longuet-Higgins 1950 & Yes & Approx. & & Yes & & Approx. \\
\hline Hasselman 1963 & Yes & & & Yes & & \\
\hline Brekhovskikh 1966 & Yes & & Yes & & & \\
\hline Hughes 1976 & Yes & & Yes & & Approx. & \\
\hline Lloyd 1981 & Yes & & Yes & & & \\
\hline Schmidt and Kuperman 1988 & Yes & & & & Yes & \\
\hline Kibblewhite and Wu 1989 & Yes & & Yes & & Approx. & \\
\hline Kibblewhite and Wu 1991,93 & Yes & Approx. & & Yes & & Approx. \\
\hline Cato 1991 & Yes & Yes & Yes & & Approx. & \\
\hline Lindstrom 1991 & Yes & & & & Yes & \\
\hline
\end{tabular}

and that the waveguide can be modeled as an infinite halfspace.

While the treatment of inhomogeneous surface wave fields and range-dependent waveguides presented here had not been previously considered, the medium nonlinear wave equation used here has a parallel derivation to the wave equations or hydrodynamic equations used in earlier works. Longuet-Higgins [78], Hasselmann [51] and Brekhovskikh [16] base their derivations on perturbing Bernoulli's equation while Hughes [54] and Kibblewhite [62] begin by separately perturbing the equations of momentum, continuity and state. We show that the non-linear wave equation used here is equivalent to perturbation expansions [54] for the physical parameters typical in hurricane microseisms. Lloyd [77] and Cato [21] base their derivations on Lighthill's equation, however, Lloyd [77] shows that both perturbation and Lighthill approaches 
yield the same end result. We also note that the non-linear wave equation can be derived as a second-order approximation of Lighthill's equation [138].

These second order non-linear or perturbation-based theories should not be confused with the linear theories proposed by Banerji [5] and Bowen et al [14]. These linear theories claim that the first-order motion of a surface gravity wave creates a first order pressure fluctuation on the sea floor regardless of how deep the ocean is. This is contrary to classic surface wave theory which shows that first-order wave motion decays exponentailly with depth such that, in deep water, the first-order pressure fluctuation on the sea floor goes to zero $[6,78,27]$ (also see App. C).

Based on the wave-height spectra in hurricane Bonnie, we calculate the microseismic source levels generated by the nonlinear interaction of the ocean surface waves. Our derivation is in agreement with earlier works $[86,78]$, which show that microseisms are generated by the non-linear interaction of ocean surface waves with roughly the same wavelength but opposing propagation directions. Recent measurements $[142,132]$ and models [88] of surface directional wave height spectra in hurricane Bonnie show the opposing surface waves necessary to generate microseisms.

\subsection{Ocean Surface Gravity Waves}

Hurricanes are characterized by high winds that can vary quickly with position, both in direction and speed, as shown in Fig. 4.2(A). In addition a hurricane may move at speeds up to $15 \mathrm{~m} / \mathrm{s}$ [33] so that the winds at any location can also change with time. These spatially and temporally varying winds generate complex ocean surface wave directional spectra (Fig. 4.2) with wave heights that can exceed $10 \mathrm{~m}[142,132,88]$.

For surface gravity waves that are homogeneous over an infinite ocean surface area we can express the complex surface wave height as the linear superposition of plane waves [68] where $\boldsymbol{\kappa}$ is the sea surface wavenumber and $\sigma$ is the corresponding frequency 

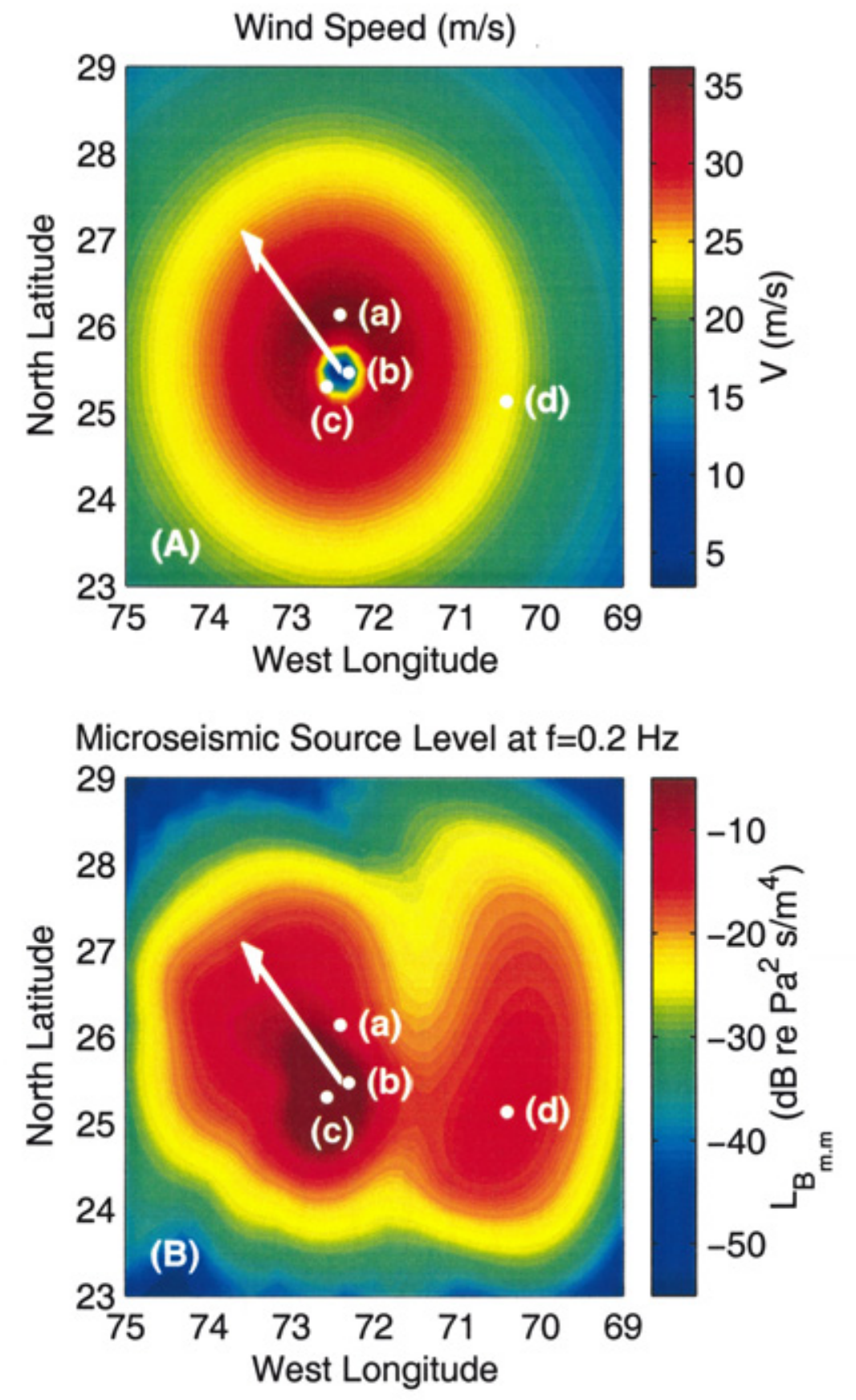

Figure 4-1: (A) Wind speed in $\mathrm{m} / \mathrm{s}$ and (B) microseismic source level $L_{B_{m m}}\left(\omega, z_{s}=0\right)$ at $\omega=1.26 \mathrm{rad} / \mathrm{s}(f=0.2 \mathrm{~Hz})$ in $\mathrm{dB}$ re $P a^{2} s / m^{4}$ from Eq. (4.39) at 1200 on 24 August 1998 as a function of latitude and longitude. The arrow indicates the direction hurricane Bonnie was moving. The letters (a), (b), (c) and (d) represent features of interest; (a) indicates the location of maximum wind speed, (b) indicates the eye where wind speed is zero, and (c) and (d) indicate peaks in the microseismic source level. This figure shows that, while a hurricane can produce significant microseismic source levels (B), these level do not 8 firectly follow the wind speeds (A) in the hurricane. 

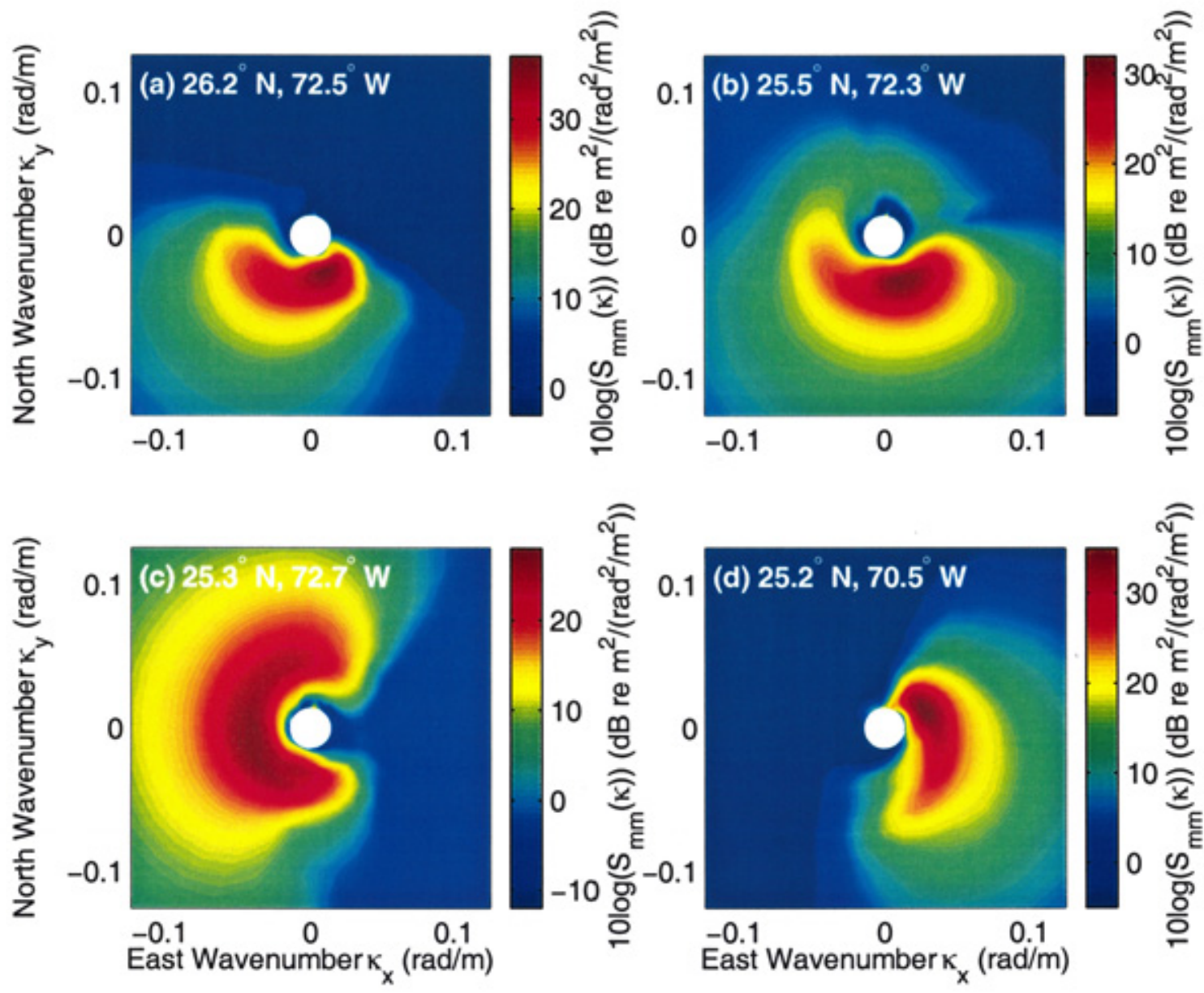

Figure 4-2: The wave-height power spectral level $\left(10 \log S_{m m}(\boldsymbol{\kappa}) /\left(m^{2} /\left(\mathrm{rad}^{2} / \mathrm{m}^{2}\right)\right)\right)$ in $\mathrm{dB}$ re $\mathrm{m}^{2} /\left(\mathrm{rad}^{2} / \mathrm{m}^{2}\right)$ at the locations of interest (a), (b), (c), and (d) given in Fig. 4.2 at 1200 on 24 August 1998. The peak in the spectra is at roughly $\kappa=0.04$ $\mathrm{rad} / \mathrm{m}$ or $\sigma=0.63 \mathrm{rad} / \mathrm{s}$ which corresponds to an acoustic frequency of $\omega=1.26$ $\mathrm{rad} / \mathrm{s}$ or $f=0.2 \mathrm{~Hz}$. At some locations (b, c, and $\mathrm{d}$ ) there are waves propagating with opposing wavenumber vectors $\kappa$, while at other locations (a), most of the waves propagate in the same direction. From Eq. (4.37) we expect these locations with opposing waves to produce the greatest microseismic source levels and shown in Fig. 4.2(B). 
where in deep water $\sigma^{2}=g \kappa$. Throughout this paper the surface wavenumber $\kappa$ is expressed either in cartesian coordinates as $\left(\kappa_{x}, \kappa_{y}\right)$ or in polar coordinates as $(\kappa, \theta)$. Also, a cartesian coordinate system is used for position where $\mathbf{r}=(\boldsymbol{\rho}, z)=(x, y, z)$ where $z$ is defined downward from the sea surface. The complex surface wave height can then be written as

$$
\hat{\zeta}(\boldsymbol{\rho}, t)=\iint_{-\infty}^{\infty} A(\boldsymbol{\kappa}) e^{-i \sigma t+i \boldsymbol{\kappa} \cdot \boldsymbol{\rho}} d^{2} \boldsymbol{\kappa}
$$

where $A(\kappa)$ is the surface wave height spectrum.

Since the complex wave height $\hat{\zeta}(\rho, t)$ is produced by the contributions of many independent random physical processes, we can assume by Central Limit Theorem that the statistical distribution of $\hat{\zeta}(\rho, t)$ is Gaussian [66]. In addition we may define the reference position from which wave height is measured such that $\hat{\zeta}(\boldsymbol{\rho}, t)$ has zero mean. Given this assumption, in App. D.1 we show that $A(\kappa)$ must also be a zeromean complex Gaussian random variable $[66,68]$.

Since the spectrum $A(\kappa)$ is zero-mean and homogeneous over an infinite ocean surface area, the second moment of the spectrum can be written as (see App. D.1)[66, $84]$

$$
<A(\kappa) A^{*}\left(\boldsymbol{\kappa}^{\prime}\right)>=S(\kappa) \delta\left(\kappa-\kappa^{\prime}\right),
$$

where $<>$ denotes the expectation. This expression shows that, for stationary processes, the wavenumber components $\kappa$ and $\kappa^{\prime}$ decorrelate. Also, since the spectrum $A(\kappa)$ is Gaussian, the fourth moment can then be written as 


$$
\begin{array}{r}
<A\left(\boldsymbol{\kappa}_{1}\right) A\left(\boldsymbol{\kappa}_{2}\right) A^{*}\left(\boldsymbol{\kappa}_{3}\right) A^{*}\left(\boldsymbol{\kappa}_{4}\right)>= \\
\delta\left(\boldsymbol{\kappa}_{1}-\boldsymbol{\kappa}_{3}\right) S\left(\boldsymbol{\kappa}_{1}\right) \delta\left(\boldsymbol{\kappa}_{2}-\boldsymbol{\kappa}_{4}\right) S\left(\boldsymbol{\kappa}_{2}\right) \\
+\delta\left(\boldsymbol{\kappa}_{1}-\boldsymbol{\kappa}_{4}\right) S\left(\boldsymbol{\kappa}_{1}\right) \delta\left(\boldsymbol{\kappa}_{2}-\boldsymbol{\kappa}_{3}\right) S\left(\boldsymbol{\kappa}_{2}\right) .
\end{array}
$$

In a hurricane the surface waves are not homogeneous over an infinite area, but rather the surface wave spectrum $A(\kappa)$ changes gradually with position in the storm. To characterize the spatially varying surface wave field in the hurricane, we divide the sea surface into a grid made up of finite regions $m$ with surface areas $S_{m}$ over which the wave spectrum $A_{m}(\kappa)$ can be taken to be homogeneous. The complex wave height in a particular finite region $m$ then can be expressed as

$$
\hat{\zeta}_{m}(\boldsymbol{\rho}, t)=\iint_{-\infty}^{\infty} A_{m}(\boldsymbol{\kappa}) e^{-i \sigma t+i \boldsymbol{\kappa} \cdot \boldsymbol{\rho}} d^{2} \boldsymbol{\kappa}
$$

In App. D.2 we show that the finite size of the regions $m$ introduces a 'windowing' effect when calculating the moments of the spectrum $A_{m}(\kappa)$. However, we also show that this windowing effect can be neglected if the dimensions $L_{x}$ and $L_{y}$ of the region are much greater than the wavelength $\lambda_{g}$ of the surface wave. For the hurricane waves of interest in this paper, the surface wavelengths $\lambda_{g}$ range from roughly 100 to $300 \mathrm{~m}$ requiring that the dimensions $L_{x}$ and $L_{y}$ be on the order of $1 \mathrm{~km}$ or greater. Provided this condition is met we can make the approximation.

$$
<A_{m}(\boldsymbol{\kappa}) A_{n}^{*}\left(\boldsymbol{\kappa}^{\prime}\right)>\approx S_{m n}(\boldsymbol{\kappa}) \delta\left(\boldsymbol{\kappa}-\boldsymbol{\kappa}^{\prime}\right)
$$

where $S_{m n}(\kappa)$ is the cross-spectral density of the surface wave fields in regions $m$ and 
$n$.

As before we can assume by Central Limit Theorem that $\hat{\zeta}_{m}(\boldsymbol{\rho}, t)$ is a zero-mean complex Gaussian random variable so that $A_{m}(\boldsymbol{\kappa})$ is also a zero-mean complex Gaussian random variable. Because of this we can write the fourth moment as

$$
\begin{array}{r}
<A_{m}\left(\boldsymbol{\kappa}_{1}\right) A_{m}\left(\boldsymbol{\kappa}_{2}\right) A_{n}^{*}\left(\boldsymbol{\kappa}_{3}\right) A_{n}^{*}\left(\boldsymbol{\kappa}_{4}\right)>\approx \\
\delta\left(\boldsymbol{\kappa}_{1}-\boldsymbol{\kappa}_{3}\right) S_{m n}\left(\boldsymbol{\kappa}_{1}\right) \delta\left(\boldsymbol{\kappa}_{2}-\boldsymbol{\kappa}_{4}\right) S_{m n}\left(\boldsymbol{\kappa}_{2}\right) \\
+\delta\left(\boldsymbol{\kappa}_{1}-\boldsymbol{\kappa}_{4}\right) S_{m n}\left(\boldsymbol{\kappa}_{1}\right) \delta\left(\boldsymbol{\kappa}_{2}-\boldsymbol{\kappa}_{3}\right) S_{m n}\left(\boldsymbol{\kappa}_{2}\right) .
\end{array}
$$

This expression for the fourth moment of the wave height spectra will be used later in Sec. 4.4 .

From Eq. (4.4) we can write the real part of the surface wave height as

$$
\zeta_{m}(\boldsymbol{\rho}, t)=\frac{1}{2} \iint_{-\infty}^{\infty}\left(A_{m}(\boldsymbol{\kappa}) e^{-i \sigma t+i \boldsymbol{\kappa} \cdot \boldsymbol{\rho}}+A_{m}^{*}(\boldsymbol{\kappa}) e^{i \sigma t-i \boldsymbol{\kappa} \cdot \boldsymbol{\rho}}\right) d^{2} \boldsymbol{\kappa}
$$

We can also express the real surface wave particle velocity $\mathbf{v}$ as a linear superposition of plane waves such that

$$
\begin{array}{r}
\mathbf{v}_{m}(\mathbf{r}, t)=\iint_{-\infty}^{\infty} \frac{-\sigma}{2}\left(\frac{\kappa_{x}}{\kappa} \hat{i}_{x}+\frac{\kappa_{y}}{\kappa} \hat{i}_{y}\right)\left(A_{m}(\boldsymbol{\kappa}) e^{-i \sigma t+i \boldsymbol{\kappa} \cdot \boldsymbol{\rho}}+A_{m}^{*}(\boldsymbol{\kappa}) e^{i \sigma t-i \boldsymbol{\kappa} \cdot \boldsymbol{\rho}}\right) e^{-\kappa z} \\
-\frac{i \sigma}{2} \hat{i}_{z}\left(A_{m}(\boldsymbol{\kappa}) e^{-i \sigma t+i \boldsymbol{\kappa} \cdot \boldsymbol{\rho}}-A_{m}^{*}(\boldsymbol{\kappa}) e^{i \sigma t-i \boldsymbol{\kappa} \cdot \boldsymbol{\rho}}\right) e^{-\kappa z} d^{2} \boldsymbol{\kappa}
\end{array}
$$

where $\hat{i}_{x}, \hat{i}_{y}$ and $\hat{i}_{z}$ represent unit vectors in the $x, y$ and $z$ directions respectively.

Wright et al [142] and Walsh et al [132] measured the spatial variation of the directional wave spectra $A_{m}(\kappa)$ in hurricane Bonnie using an aircraft-mounted Scanning 
Radar Altimeter (SRA). These measurements, however, are limited to the locations and times of the aircraft flights and do not give a complete picture of the wave field in the hurricane.

To quantify the surface wave field at any location and time in hurricane Bonnie, Moon et al [88] used the Wave Watch III (WW3) ocean surface wave model [126]. These model results, shown in Fig. 4.2, are in close agreement with measurements from Wright et al [142] and Walsh et al [132] and with data from bouys and oceanographic stations. In this paper we use the surface directional wave spectra $A_{m}(\kappa)$ in hurricane Bonnie calculated using WW3 [88].

Experiments by Forristall [42] show that the linear wave theory of Eqs. (4.1, 4.4, 4.7 and 4.8 ) is adequate to describe surface waves even in high sea states with significant wave breaking as in a hurricane. The use of linear surface wave theory can also be justified since the typical surface wave heights in a hurricane are an order of magnitude less than the wavelength $[42,142,132,88]$.

\subsection{Nonlinear Wave Equation}

The non-linear wave equation $[137,28]$ describes the second-order acoustic wave field generated by first-order fluid motion. For the long surface waves in a hurricane viscosity can be neglected so that the non-linear wave equation can be written as [89]

$$
\begin{array}{r}
\nabla^{2} p_{2}(\mathbf{r}, t)-\frac{1}{c^{2}} \frac{\partial^{2} p_{2}(\mathbf{r}, t)}{\partial t^{2}}=\frac{\Gamma}{\rho c^{4}} \frac{\partial^{2} p_{1}^{2}(\mathbf{r}, t)}{\partial t^{2}} \\
-\frac{\rho}{2 c^{2}} \frac{\partial^{2}}{\partial t^{2}}\left(\mathbf{v}_{1}(\mathbf{r}, t) \cdot \mathbf{v}_{1}(\mathbf{r}, t)\right) \\
-\frac{\rho}{2} \nabla^{2}\left(\mathbf{v}_{1}(\mathbf{r}, t) \cdot \mathbf{v}_{1}(\mathbf{r}, t)\right),
\end{array}
$$

where the first order velocity $\mathbf{v}_{1}$ and pressure $p_{1}$ terms on the right hand side generate 
a second order acoustic pressure field $p_{2}$ on the left hand side, $c$ is the sound speed, $\rho$ is density and $\Gamma$ is the coefficient of nonlinearity $[28,89]$.

In this work, we consider the case where the first order velocity $\mathbf{v}_{1}$, from Eq. (4.8), is due to surface wave motion. The first order pressure $p_{1}$ can be found using conservation of momentum where $-\nabla p_{1}=\rho \partial \mathbf{v}_{1} / \partial t$. Using the relationship between first-order velocity and pressure we can compare the relative magnitudes of the source terms in Eq. (4.9). Given typical parameters $\kappa=0.02$ to $0.7 \mathrm{rad} / \mathrm{m}, \sigma=0.4$ to $0.8 \mathrm{rad} / \mathrm{s}, c \approx 1500 \mathrm{~m} / \mathrm{s}, \rho \approx 1000 \mathrm{~kg} / \mathrm{m}^{3}$, and $\Gamma \approx-2.6$ [13] we find that the $\frac{\rho_{0}}{2} \nabla^{2}\left(\mathbf{v}_{1}(\mathbf{r}, t) \cdot \mathbf{v}_{1}(\mathbf{r}, t)\right)$ term of Eq. (4.9) exceeds the other source terms by several orders of magnitude. Dropping the lesser terms yields

$$
\nabla^{2} p_{2}(\mathbf{r}, t)-\frac{1}{c^{2}} \frac{\partial^{2} p_{2}(\mathbf{r}, t)}{\partial t^{2}}=-\frac{\rho}{2} \nabla^{2}\left(\mathbf{v}_{1}(\mathbf{r}, t) \cdot \mathbf{v}_{1}(\mathbf{r}, t)\right)=q(\mathbf{r}, t)
$$

This equation is equivalent to the perturbation expression used in the previous microseism derivation of Hughes [54]. This can be seen by taking Eq. (10) of Hughes [54] and assuming irrotational flow $\left(\nabla\left(\mathbf{v}_{1}(\mathbf{r}, t) \cdot \mathbf{v}_{1}(\mathbf{r}, t)\right)=2\left(\mathbf{v}_{1}(\mathbf{r}, t) \cdot \nabla\right) \mathbf{v}_{1}(\mathbf{r}, t)\right)$ and continuity $\left(\nabla \cdot\left(\rho_{0} \mathbf{v}_{1}(\mathbf{r}, t)\right)=0\right)$. The assumption of irrotational flow is justifiable for linear ocean surface wave fields.

Taking the Fourier Transform of Eq. (4.10) we find the frequency domain Helmholtz equation for the second order field

$$
\nabla^{2} P_{2}(\mathbf{r}, \omega)+k^{2} P_{2}(\mathbf{r}, \omega)=q(\mathbf{r}, \omega)
$$

We will begin by solving for the pressure field generated by a finite volume $V_{m}$ with a source distribution $q_{m}$ which depends on the fluid velocity $\mathbf{v}_{1, m}$ from Eq. (4.8). The volume $V_{m}$ extends vertically from just below the ocean surface at $z=0$ to a 
depth below the surface wave region $\left(z>\lambda_{g}=2 \pi / \kappa\right)$. The volume extends over the horizontal area $S_{m}$ with dimensions $L_{x}$ and $L_{y}$ centered at $\boldsymbol{\rho}_{m}=\left(x_{m}, y_{m}\right)$ as defined in Sec. 4.2. We can solve the Helmholtz Equation (Eq. (4.11)), using the frequency domain Green function $g\left(\mathbf{r}_{r}, \mathbf{r}_{s}, \omega\right)$ such that,

$$
P_{2, m}\left(\mathbf{r}_{r}, \omega\right)=\iiint_{V_{m}} g\left(\mathbf{r}_{r}, \mathbf{r}_{s}, \omega\right) q_{m}\left(\mathbf{r}_{s}, \omega\right) d^{3} \mathbf{r}_{s}
$$

where $\mathbf{r}_{s}=\left(x_{s}, y_{s}, z_{s}\right)$ and $\mathbf{r}_{r}=\left(x_{r}, y_{r}, z_{r}\right)$ are the source and receiver locations respectively.

$$
P_{2, m}\left(\mathbf{r}_{r}, w\right)=\int_{0}^{\lambda_{g}} d z_{s} \iint_{-\infty}^{\infty} w\left(\boldsymbol{\rho}_{s}-\boldsymbol{\rho}_{m}\right) g\left(\mathbf{r}_{r}, \mathbf{r}_{s}, w\right) q_{m}\left(\mathbf{r}_{s}, w\right) d^{2} \boldsymbol{\rho}_{s}
$$

where the window function $w\left(\boldsymbol{\rho}_{s}-\boldsymbol{\rho}_{m}\right)$ is unity for $-L_{x} / 2<x_{s}-x_{m}<L_{x} / 2$ and $-L_{y} / 2<y_{s}-y_{m}<L_{y} / 2$ and zero elsewhere. The double integral over $\boldsymbol{\rho}_{s}$ represents the field at a receiver $\mathbf{r}_{r}$ due to a distribution of sources $q$ over a finite area defined by the window function $w$. In effect this finite area acts as a horizontal planar array. If the receiver $\mathbf{r}_{r}$ is in the far field of the finite area $\left(\left|\mathbf{r}_{r}-\mathbf{r}_{m}\right|>L_{x}^{2} / \lambda_{a}\right.$ and $\left|\mathbf{r}_{r}-\mathbf{r}_{m}\right|>$ $L_{y}^{2} / \lambda_{a}$ where $\lambda_{a}$ is the acoustic wavelength and where $\left.\mathbf{r}_{m}=\left(\boldsymbol{\rho}_{m}, z_{s}\right)=\left(x_{m}, y_{m}, z_{s}\right)\right)$, we can make the plane wave approximation,

$$
g\left(\mathbf{r}_{r}, \mathbf{r}_{s}, w\right) \approx g\left(\mathbf{r}_{r}, \mathbf{r}_{m}, w\right) e^{-i \mathbf{k}_{r} \cdot\left(\boldsymbol{\rho}_{s}-\boldsymbol{\rho}_{m}\right)}
$$

where the acoustic wavenumber is defined in cartesian coordinates as $\mathbf{k}=\left(\mathbf{k}_{r}, k_{z}\right)=$ $\left(k_{x}, k_{y}, k_{z}\right)$ where $\omega / c=\sqrt{k_{x}^{2}+k_{y}^{2}+k_{z}^{2}}=k$. In free space this would simplify to 


$$
g\left(\mathbf{r}_{r}, \mathbf{r}_{s}, w\right)=\frac{1}{4 \pi\left|\boldsymbol{\rho}_{r}-\boldsymbol{\rho}_{s}\right|} e^{i k\left|\boldsymbol{\rho}_{r}-\boldsymbol{\rho}_{s}\right|} \approx \frac{1}{4 \pi\left|\boldsymbol{\rho}_{r}-\boldsymbol{\rho}_{m}\right|} e^{i k\left|\boldsymbol{\rho}_{r}-\boldsymbol{\rho}_{m}\right|} e^{-i \mathbf{k}_{r} \cdot\left(\boldsymbol{\rho}_{s}-\boldsymbol{\rho}_{m}\right)}
$$

however, we will use the more general form given in Eq. (4.14). Inserting Eq. (4.14) into Eq. (4.13) yields

$$
P_{2, m}\left(\mathbf{r}_{r}, w\right) \approx \int_{0}^{\lambda_{g}} d z_{s} g\left(\mathbf{r}_{r}, \mathbf{r}_{m}, w\right) \iint_{-\infty}^{\infty} w\left(\boldsymbol{\rho}_{s}-\boldsymbol{\rho}_{m}\right) e^{-i \mathbf{k}_{r} \cdot\left(\boldsymbol{\rho}_{s}-\boldsymbol{\rho}_{m}\right)} q_{m}\left(\mathbf{r}_{s}, w\right) d^{2} \boldsymbol{\rho}_{s}
$$

Again, the double integral over $\rho_{s}$ analogous to a horizontal planar source array with broadside directed down toward the ocean bottom. Following this analogy we can define this double integral as the beamformed output [129]

$$
B_{m}\left(\mathbf{k}, z_{s}\right)=\iint_{-\infty}^{\infty} w\left(\boldsymbol{\rho}_{s}-\boldsymbol{\rho}_{m}\right) e^{-i \mathbf{k}_{r} \cdot\left(\boldsymbol{\rho}_{s}-\boldsymbol{\rho}_{m}\right)} q_{m}\left(\mathbf{r}_{s}, w\right) d^{2} \boldsymbol{\rho}_{s}
$$

The beamformed output in Eq. (4.17) can be evaluated for any horizontal wavenumber vector $\mathbf{k}_{r}$, however, only those wavenumber vectors where $\left|\mathbf{k}_{\mathbf{r}}\right| \leq \omega / c$ lead to a propagating acoustic field. This can be illustrated if we consider the microseismic field generated by a horizontal plane of sources near the sea surface as described in Eq. (4.17). In previous works [78] it has been shown that microseisms generated at the ocean surface are first transmitted downward to the sea floor where they then propagate along the bottom as Rayleigh waves. This vertical propagation from the sea surface to the sea floor can be modeled to first order using the free-space Green function 


$$
g(w)=\frac{1}{4 \pi d} e^{i k_{z} d}
$$

where $d$ represents the water column depth. If $\left|\mathbf{k}_{\mathbf{r}}\right|$ exceeds $\omega / c$ then $k_{z}$ becomes imaginary leading to an exponential decay with depth $d$. For example if $\left|\mathbf{k}_{\mathbf{r}}\right|=2 \omega / c$ then $k_{z}=i \sqrt{3} \omega / c$ so that, for $\omega=1.3 \mathrm{rad} / \mathrm{s}, c=1500 \mathrm{~m} / \mathrm{s}$ and an ocean depth $d=1000 \mathrm{~m}$, the field is attenuated by $e^{-k_{z} z_{r}} \approx 0.2$ or $13 \mathrm{~dB}$. Greater depths will lead to even more attenuation. Because of this evanescent attenuation we will only consider the acoustic field where $\left|\mathbf{k}_{\mathbf{r}}\right| \leq \omega / c$.

Assuming the acoustic pressure field given in Eq. (4.16) is temporally stationary, the power spectral density may be written as [91]

$$
\begin{aligned}
S_{P_{m, n}}\left(\mathbf{r}_{r}, \omega\right) \delta\left(\omega-\omega^{\prime}\right)= & <P_{2, m}\left(\mathbf{r}_{r}, w\right) P_{2, n}^{*}\left(\mathbf{r}_{r}, w^{\prime}\right)> \\
\approx & \int_{0}^{\lambda_{g}} \int_{0}^{\lambda_{g}} d z_{s} d z_{s}^{\prime} g\left(\mathbf{r}_{r}, \mathbf{r}_{m}, w\right) g^{*}\left(\mathbf{r}_{r}, \mathbf{r}_{n}, w^{\prime}\right) \\
& \times<B_{m}\left(\mathbf{k}, z_{s, m}\right) B_{n}^{*}\left(\mathbf{k}^{\prime}, z_{s, n}\right)>,
\end{aligned}
$$

where $<>$ represents the expectation and $\mathbf{r}_{n}=\left(\boldsymbol{\rho}_{n}, z_{s}^{\prime}\right)=\left(x_{n}, y_{n}, z_{s}^{\prime}\right)$. In the next section we derive expressions for the second moment $<B_{m}\left(\mathbf{k}, z_{s, m}\right) B_{n}^{*}\left(\mathbf{k}^{\prime}, z_{s, n}\right)>$ of the beamformed output and the power spectral density $S_{P_{m, n}}$ due to ocean surface waves.

The power spectral density $S_{P_{m}, n}$ in Eq. (4.19) represents the field at a receiver from a single pair of finite volumes $V_{m}$ and $V_{n}$. In the next section we determine the microseismic field generated by a hurricane where the surface wave field is inhomogeneous and extends over a large region hundreds of kilometers across. To do this we divide the hurricane region into finite volumes $V_{m}$, as shown in Fig. 4.3, and then 
sum their contributions to find the total power spectral density of the received field written as

$$
S_{P}\left(\mathbf{r}_{r}, \omega\right)=\sum_{m, n} S_{P_{m, n}}\left(\mathbf{r}_{r}, \omega\right) .
$$

\subsection{Power Spectral Density due to Ocean Surface Gravity Waves}

Taking the ocean surface wave velocity as the first-order field we can determine the source term from Eqs. (4.11) where $q\left(\mathbf{r}_{s}, \omega\right)$ is the Fourier transform

$$
q_{m}\left(\mathbf{r}_{s}, \omega\right)=\frac{1}{2 \pi} \int_{-\infty}^{\infty} q_{m}\left(\mathbf{r}_{s}, t\right) e^{i w t} d t
$$

of

$$
q_{m}\left(\mathbf{r}_{s}, t\right)=-\frac{\rho}{2} \nabla^{2}\left(\mathbf{v}_{1, m}\left(\mathbf{r}_{s}, t\right) \cdot \mathbf{v}_{1, m}\left(\mathbf{r}_{s}, t\right)\right)
$$

Given Eq. (4.8) for the first order velocity we find

$$
\begin{aligned}
\nabla^{2}\left(\mathbf{v}_{1, m}\left(\mathbf{r}_{s}, t\right) \cdot \mathbf{v}_{1, m}\left(\mathbf{r}_{s}, t\right)\right)= & \iint_{-\infty}^{\infty} \iint_{-\infty}^{\infty} \frac{\sigma_{1} \sigma_{2}}{4} \\
& \times\left[A_{m}\left(\boldsymbol{\kappa}_{1}\right) A_{m}\left(\boldsymbol{\kappa}_{2}\right) e^{-i \sigma^{+} t} e^{i \boldsymbol{\kappa}^{+} \cdot \boldsymbol{\rho}_{s}} F_{1}\left(\boldsymbol{\kappa}_{1}, \boldsymbol{\kappa}_{2}\right)\right. \\
& +A_{m}^{*}\left(\boldsymbol{\kappa}_{1}\right) A_{m}^{*}\left(\boldsymbol{\kappa}_{2}\right) e^{i \sigma^{+} t} e^{-i \boldsymbol{\kappa}^{+} \cdot \boldsymbol{\rho}_{s} F_{1}\left(\boldsymbol{\kappa}_{1}, \boldsymbol{\kappa}_{2}\right)} \\
& +A_{m}\left(\boldsymbol{\kappa}_{1}\right) A_{m}^{*}\left(\boldsymbol{\kappa}_{2}\right) e^{-i \sigma^{-} t} e^{i \boldsymbol{\kappa}^{-} \cdot \boldsymbol{\rho}_{s} F_{2}\left(\boldsymbol{\kappa}_{1}, \boldsymbol{\kappa}_{2}\right)}
\end{aligned}
$$




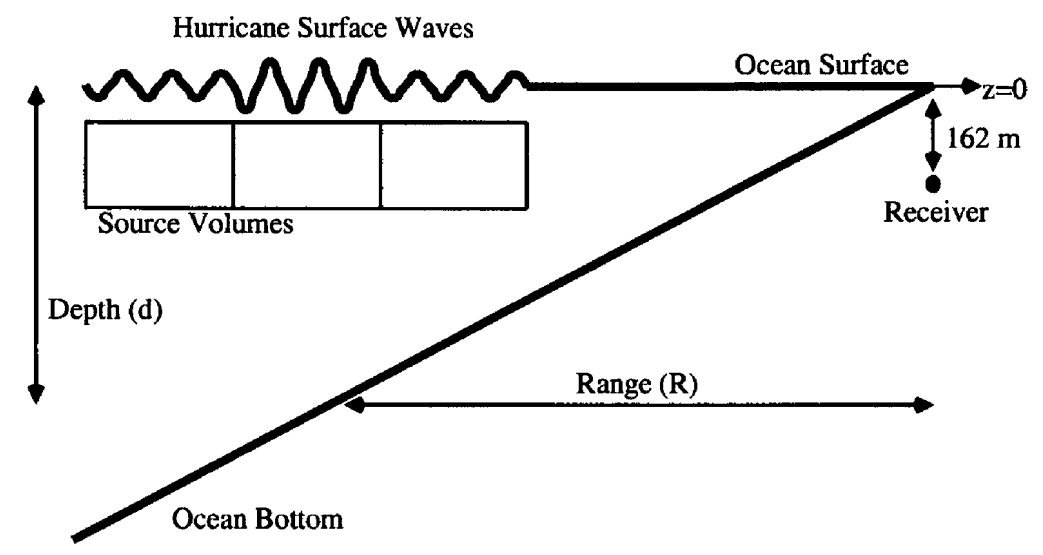

Figure 4-3: Geometry of the hurricane wave field and ocean waveguide (not to scale). In our model the waveguide environment may be range dependent and in this paper we consider the example of an upslope propagation from the deep North Atlantic to Florida. The range and ocean depth parameters $\mathrm{R}$ and $\mathrm{d}$ are given in Table 4.6. The depth of the receiver below the ocean bottom of $162 \mathrm{~m}$ corresponds to the depth below the earth surface of the actual seismometer in Florida. The compression wave speeds $c_{p}$ are 1500 and $5200 \mathrm{~m} / \mathrm{s}$ in the water and bottom respectively. The shear wave speed $c_{s}$ in the bottom is $3000 \mathrm{~m} / \mathrm{s}$. The densities $\rho$ in the water and bottom are 1.0 and $2.5 \mathrm{~g} / \mathrm{cm}$ respectively. 


$$
\begin{aligned}
& +A_{m}^{*}\left(\boldsymbol{\kappa}_{1}\right) A_{m}\left(\boldsymbol{\kappa}_{2}\right) e^{i \sigma^{-} t} e^{\left.-i \boldsymbol{\kappa}^{-} \cdot \boldsymbol{\rho}_{s} F_{2}\left(\boldsymbol{\kappa}_{1}, \boldsymbol{\kappa}_{2}\right)\right]} \\
& \times e^{-\left(\kappa_{1}+\kappa_{2}\right) z_{s}} d^{2} \kappa_{1} d^{2} \kappa_{2}
\end{aligned}
$$

where $\sigma^{+}=\left(\sigma_{1}+\sigma_{2}\right), \sigma^{-}=\left(\sigma_{1}-\sigma_{2}\right), \boldsymbol{\kappa}^{+}=\left(\kappa_{1}+\boldsymbol{\kappa}_{2}\right)$, and $\boldsymbol{\kappa}^{-}=\left(\boldsymbol{\kappa}_{1}-\boldsymbol{\kappa}_{2}\right)$. Note that the Laplacian operator $\left(\nabla^{2}\right)$ in Eq. (4.23) leads to the direction cosine functions

$$
\begin{aligned}
F_{1}\left(\kappa_{1}, \kappa_{2}\right) & =\left[\left(\kappa^{+}\right)^{2}-\left(\kappa_{x}^{+}\right)^{2}-\left(\kappa_{y}^{+}\right)^{2}\right]\left[-1+\frac{\kappa_{1 x} \kappa_{2 x}}{\kappa_{1} \kappa_{2}}+\frac{\kappa_{1 y} \kappa_{2 y}}{\kappa_{1} \kappa_{2}}\right] \\
& =2 \kappa_{1} \kappa_{2}\left[1-\cos \left(\theta_{1}-\theta_{2}\right)\right]\left[-1+\cos \left(\theta_{1}-\theta_{2}\right)\right]
\end{aligned}
$$

and

$$
\begin{aligned}
F_{2}\left(\kappa_{1}, \kappa_{2}\right) & =\left[\left(\kappa^{-}\right)^{2}-\left(\kappa_{x}^{-}\right)^{2}-\left(\kappa_{y}^{-}\right)^{2}\right]\left[1+\frac{\kappa_{1 x} \kappa_{2 x}}{\kappa_{1} \kappa_{2}}+\frac{\kappa_{1 y} \kappa_{2 y}}{\kappa_{1} \kappa_{2}}\right] \\
& =2 \kappa_{1} \kappa_{2}\left[-1+\cos \left(\theta_{1}-\theta_{2}\right)\right]\left[1+\cos \left(\theta_{1}-\theta_{2}\right)\right]
\end{aligned}
$$

where $\kappa_{x}^{+}=\left(\kappa_{1 x}+\kappa_{2 x}\right), \kappa_{y}^{+}=\left(\kappa_{1 y}+\kappa_{2 y}\right), \kappa_{x}^{-}=\left(\kappa_{1 x}-\kappa_{2 x}\right)$, and $\kappa_{y}^{-}=\left(\kappa_{1 y}-\kappa_{2 y}\right)$.

Substituting Eq. (4.23) into the definition of $q_{m}\left(\mathbf{r}_{s}, t\right)$ from Eq. (4.22) and taking the Fourier transform yields,

$$
\begin{aligned}
q_{m}\left(\mathbf{r}_{s}, \omega\right)= & -\frac{\rho}{8} \iint_{-\infty}^{\infty} \iint_{-\infty}^{\infty} \sigma_{1} \sigma_{2} \\
& \times\left[A_{m}\left(\boldsymbol{\kappa}_{1}\right) A_{m}\left(\boldsymbol{\kappa}_{2}\right) F_{1}\left(\boldsymbol{\kappa}_{1}, \boldsymbol{\kappa}_{2}\right) \delta\left(\omega-\sigma^{+}\right) e^{i \boldsymbol{\kappa}^{+} \cdot \boldsymbol{\rho}_{s}}\right. \\
& +A_{m}^{*}\left(\boldsymbol{\kappa}_{1}\right) A_{m}^{*}\left(\boldsymbol{\kappa}_{2}\right) F_{1}\left(\boldsymbol{\kappa}_{1}, \boldsymbol{\kappa}_{2}\right) \delta\left(\omega+\sigma^{+}\right) e^{-i \boldsymbol{\kappa}^{+} \cdot \boldsymbol{\rho}_{s}} \\
& +A_{m}\left(\boldsymbol{\kappa}_{1}\right) A_{m}^{*}\left(\boldsymbol{\kappa}_{2}\right) F_{2}\left(\boldsymbol{\kappa}_{1}, \boldsymbol{\kappa}_{2}\right) \delta\left(\omega-\sigma^{-}\right) e^{i \boldsymbol{\kappa}^{-} \cdot \boldsymbol{\rho}_{s}} \\
& \left.+A_{m}^{*}\left(\boldsymbol{\kappa}_{1}\right) A_{m}\left(\boldsymbol{\kappa}_{2}\right) F_{2}\left(\boldsymbol{\kappa}_{1}, \boldsymbol{\kappa}_{2}\right) \delta\left(\omega+\sigma_{1}^{-}\right) e^{-i \boldsymbol{\kappa}^{-} \cdot \boldsymbol{\rho}_{s}}\right] \\
& \times e^{-\left(\kappa_{1}+\kappa_{2}\right) z_{s}} d^{2} \boldsymbol{\kappa}_{1} d^{2} \boldsymbol{\kappa}_{2} .
\end{aligned}
$$


By inserting Eq. (4.26) into the definition of the beamformed output $B_{m}\left(\mathbf{k}, z_{s}\right)$ from Eq. (4.17) we obtain

$$
\begin{aligned}
B_{m}\left(\mathbf{k}, z_{s}\right)= & -\frac{\rho}{8} \iint_{-\infty}^{\infty} \iint_{-\infty}^{\infty} \sigma_{1} \sigma_{2} \\
& \times\left[A_{m}\left(\boldsymbol{\kappa}_{1}\right) A_{m}\left(\boldsymbol{\kappa}_{2}\right) F_{1}\left(\boldsymbol{\kappa}_{1}, \boldsymbol{\kappa}_{2}\right) \delta\left(\omega-\sigma^{+}\right)\right. \\
& \times\left(\int_{-\infty}^{\infty} \int_{-\infty}^{\infty} w\left(\boldsymbol{\rho}_{s}-\boldsymbol{\rho}_{m}\right) e^{i \boldsymbol{\kappa}^{+} \cdot \boldsymbol{\rho}_{s}} e^{-i \mathbf{k}_{r} \cdot\left(\boldsymbol{\rho}_{s}-\boldsymbol{\rho}_{m}\right)} d^{2} \boldsymbol{\rho}_{s}\right) \\
& +A_{m}^{*}\left(\boldsymbol{\kappa}_{1}\right) A_{m}^{*}\left(\boldsymbol{\kappa}_{2}\right) F_{1}\left(\boldsymbol{\kappa}_{1}, \boldsymbol{\kappa}_{2}\right) \delta\left(\omega+\left(\sigma_{1}+\sigma_{2}\right)\right) \\
& \times\left(\int_{-\infty}^{\infty} \int_{-\infty}^{\infty} w\left(\boldsymbol{\rho}_{s}-\boldsymbol{\rho}_{m}\right) e^{-i\left(\boldsymbol{\kappa}_{1}+\boldsymbol{\kappa}_{2}\right) \cdot \boldsymbol{\rho}_{s}} e^{-i \mathbf{k}_{H} \cdot\left(\boldsymbol{\rho}_{s}-\boldsymbol{\rho}_{m}\right)} d^{2} \boldsymbol{\rho}_{s}\right) \\
& +A_{m}^{*}\left(\boldsymbol{\kappa}_{1}\right) A_{m}\left(\boldsymbol{\kappa}_{2}\right) F_{2}\left(\boldsymbol{\kappa}_{1}, \boldsymbol{\kappa}_{2}\right) \delta\left(\omega-\left(\sigma_{1}-\sigma_{2}\right)\right) \\
& \times\left(\int_{-\infty}^{\infty} \int_{-\infty}^{\infty} w\left(\boldsymbol{\rho}_{s}-\boldsymbol{\rho}_{m}\right) e^{i\left(\boldsymbol{\kappa}_{1}-\boldsymbol{\kappa}_{2}\right) \cdot \boldsymbol{\rho}_{s}} e^{-i \mathbf{k}_{H} \cdot\left(\boldsymbol{\rho}_{s}-\boldsymbol{\rho}_{m}\right)} d^{2} \boldsymbol{\rho}_{s}\right) \\
& +A_{m}^{*}\left(\boldsymbol{\kappa}_{1}\right) A_{m}\left(\boldsymbol{\kappa}_{2}\right) F_{2}\left(\boldsymbol{\kappa}_{1}, \boldsymbol{\kappa}_{2}\right) \delta\left(\omega+\left(\sigma_{1}-\sigma_{2}\right)\right) \\
& \left.\times\left(\int_{-\infty}^{\infty} \int_{-\infty}^{\infty} w\left(\boldsymbol{\rho}_{s}-\boldsymbol{\rho}_{m}\right) e^{-i\left(\boldsymbol{\kappa}_{1}-\boldsymbol{\kappa}_{2}\right) \cdot \boldsymbol{\rho}_{s}} e^{-i \mathbf{k}_{H} \cdot\left(\boldsymbol{\rho}_{s}-\boldsymbol{\rho}_{m}\right)} d^{2} \boldsymbol{\rho}_{s}\right)\right] \\
& \times e^{-\left(\kappa_{1}+\boldsymbol{\kappa}_{2}\right) z_{s}} d^{2} \boldsymbol{\kappa}_{1} d^{2} \boldsymbol{\kappa}_{2} .
\end{aligned}
$$

Note that the wave height spectra $A_{m}$ and $A_{n}$ in Eq. (4.27) can be brought out of the spatial integral since they are constant over the ocean surface area $S_{m}$ defined by the window function $w$. Integrating over $\boldsymbol{\rho}_{s}$ then leads to

$$
\begin{aligned}
B_{m}\left(\mathbf{k}, z_{s}\right)= & -\frac{\rho}{8} \iint_{-\infty}^{\infty} \iint_{-\infty}^{\infty} \sigma_{1} \sigma_{2} \\
& \times\left[A_{m}\left(\boldsymbol{\kappa}_{1}\right) A_{m}\left(\boldsymbol{\kappa}_{2}\right) F_{1}\left(\boldsymbol{\kappa}_{1}, \boldsymbol{\kappa}_{2}\right) \delta\left(\omega-\sigma^{+}\right)\left(W\left(\mathbf{k}_{r}\right) * \delta\left(\mathbf{k}_{r}-\boldsymbol{\kappa}^{+}\right)\right)\right. \\
& +A_{m}^{*}\left(\boldsymbol{\kappa}_{1}\right) A_{m}^{*}\left(\boldsymbol{\kappa}_{2}\right) F_{1}\left(\boldsymbol{\kappa}_{1}, \boldsymbol{\kappa}_{2}\right) \delta\left(\omega+\sigma^{+}\right)\left(W\left(\mathbf{k}_{r}\right) * \delta\left(\mathbf{k}_{r}+\boldsymbol{\kappa}^{+}\right)\right) \\
& +A_{m}\left(\boldsymbol{\kappa}_{1}\right) A_{m}^{*}\left(\boldsymbol{\kappa}_{2}\right) F_{2}\left(\boldsymbol{\kappa}_{1}, \boldsymbol{\kappa}_{2}\right) \delta\left(\omega-\sigma^{-}\right)\left(W\left(\mathbf{k}_{r}\right) * \delta\left(\mathbf{k}_{r}-\boldsymbol{\kappa}^{-}\right)\right)
\end{aligned}
$$




$$
\begin{aligned}
& \left.+A_{m}^{*}\left(\boldsymbol{\kappa}_{1}\right) A_{m}\left(\boldsymbol{\kappa}_{2}\right) F_{2}\left(\boldsymbol{\kappa}_{1}, \boldsymbol{\kappa}_{2}\right) \delta\left(\omega+\sigma^{-}\right)\left(W\left(\mathbf{k}_{r}\right) * \delta\left(\mathbf{k}_{r}+\boldsymbol{\kappa}^{-}\right)\right)\right] \\
& \times e^{-\left(\kappa_{1}+\kappa_{2}\right) z_{s}} d^{2} \boldsymbol{\kappa}_{1} d^{2} \boldsymbol{\kappa}_{2},
\end{aligned}
$$

where $*$ represents the two-dimensional convolution written as,

$$
F\left(\mathbf{k}_{r}\right) * G\left(\mathbf{k}_{r}\right)=\iint_{-\infty}^{\infty} F\left(\mathbf{k}_{r}^{\prime}\right) G\left(\mathbf{k}_{r}-\mathbf{k}_{r}^{\prime}\right) d^{2} \mathbf{k}_{r}^{\prime}
$$

and where

$$
W\left(\mathbf{k}_{r}\right)=\frac{2 \sin \left(\frac{k_{x} L_{x}}{2}\right)}{k_{x}} \frac{2 \sin \left(\frac{k_{y} L_{y}}{2}\right)}{k_{y}} .
$$

Note that, when integrating over $\boldsymbol{\rho}_{s}$, the $\boldsymbol{\rho}_{m}$ term in the window function $w\left(\boldsymbol{\rho}_{s}-\boldsymbol{\rho}_{m}\right)$ of Eq. (4.27) introduces a phase factor $e^{-i \mathbf{k}_{r} \cdot \boldsymbol{\rho}_{m}}$. This cancels with the other phase term $e^{i \mathbf{k}_{r} \cdot \boldsymbol{\rho}_{m}}$ in Eq. (4.27). From this we see that the source beamformed output given in Eq. (4.28) has no phase dependence on location $\boldsymbol{\rho}_{m}$.

The delta functions in Eq. (4.28) show that the horizontal component $\mathbf{k}_{r}$ of the acoustic wavenumber vector is generated by and equals either the sum $\kappa^{+}$or difference $\kappa^{-}$of the surface wavenumber. In Section 4.3 we discussed how the non-propagating field where $\left|\mathbf{k}_{r}\right|>\frac{\omega}{c}$ is not significant and may be ignored. Therefore, when integrating over $\kappa_{2}$ we need only consider a range of integration $\Omega$ corresponding to propagating acoustic waves. For the sum terms in Eq. (4.28) $\Omega$ corresponds to the integration region where $\left|\kappa^{+}\right| \leq \frac{\omega}{c}$ and for the difference terms $\Omega$ corresponds to $\left|\kappa^{-}\right| \leq \frac{\omega}{c}$.

Here we will define the source area dimensions $L_{x}$ and $L_{y}$ to be 'acoustically small' $\left(L_{x}, L_{y}<<\lambda_{a}\right)$. The acoustic wavelengths of the microseismic field in water range from roughly 6 to $11 \mathrm{~km}$ requiring that the dimensions $L_{x}$ and $L_{y}$ be on the order of 1 $\mathrm{km}$ or less. Note that this requirement on $L_{x}$ and $L_{y}$, combined with the requirement 
that $L_{x}, L_{y}>>\lambda_{g}$ from Sec. 4.2 , means that $\lambda_{g}<<L_{x}, L_{y}<<\lambda_{a}$. If $L_{x}, L_{y}<<\lambda_{a}$, the function $W\left(\mathbf{k}_{r}\right) \approx L_{x} L_{y}$ in Eq. (4.30) within the range of acoustically propagating wavenumbers $\mathbf{k}_{r} \leq \omega / c$. With this approximation Eq (4.28) can be written as

$$
\begin{aligned}
B_{m}\left(\mathbf{k}, z_{s}\right)= & -\frac{\rho L_{x} L_{y}}{8} \iint_{-\infty}^{\infty} \iint_{\Omega} \sigma_{1} \sigma_{2} \\
& \times\left[A_{m}\left(\kappa_{1}\right) A_{m}\left(\boldsymbol{\kappa}_{2}\right) F_{1}\left(\boldsymbol{\kappa}_{1}, \boldsymbol{\kappa}_{2}\right) \delta\left(\omega-\sigma^{+}\right)\right. \\
& +A_{m}^{*}\left(\boldsymbol{\kappa}_{1}\right) A_{m}^{*}\left(\boldsymbol{\kappa}_{2}\right) F_{1}\left(\boldsymbol{\kappa}_{1}, \boldsymbol{\kappa}_{2}\right) \delta\left(\omega+\sigma^{+}\right) \\
& +A_{m}\left(\boldsymbol{\kappa}_{1}\right) A_{m}^{*}\left(\boldsymbol{\kappa}_{2}\right) F_{2}\left(\boldsymbol{\kappa}_{1}, \boldsymbol{\kappa}_{2}\right) \delta\left(\omega-\sigma^{-}\right) \\
& \left.+A_{m}^{*}\left(\boldsymbol{\kappa}_{1}\right) A_{m}\left(\boldsymbol{\kappa}_{2}\right) F_{2}\left(\boldsymbol{\kappa}_{1}, \boldsymbol{\kappa}_{2}\right) \delta\left(\omega+\sigma^{-}\right)\right] \\
& \times e^{-\left(\kappa_{1}+\kappa_{2}\right) z_{s}} d^{2} \boldsymbol{\kappa}_{1} d^{2} \boldsymbol{\kappa}_{2} .
\end{aligned}
$$

The power spectral density of the pressure in Eq. (4.19) contains the second moment or variance of the beamformed output $B_{m}\left(\mathbf{k}, z_{s}\right)$ which from Eq. (4.31) can be expressed as,

$$
\begin{aligned}
<B_{m}\left(\mathbf{k}, z_{s}\right) B_{n}^{*}\left(\mathbf{k}^{\prime}, z_{s}^{\prime}\right)>= & \frac{\rho^{2} L_{x}^{2} L_{y}^{2}}{64} \iint_{-\infty}^{\infty} \iint_{\Omega} \iint_{-\infty}^{\infty} \iint_{\Omega} \sigma_{1} \sigma_{2} \sigma_{3} \sigma_{4} \\
& \times<\left[A_{m}\left(\boldsymbol{\kappa}_{1}\right) A_{m}\left(\boldsymbol{\kappa}_{2}\right) F_{1}\left(\boldsymbol{\kappa}_{1}, \boldsymbol{\kappa}_{2}\right) \delta\left(\omega-\left(\sigma_{1}+\sigma_{2}\right)\right)\right. \\
& +A_{m}^{*}\left(\boldsymbol{\kappa}_{1}\right) A_{m}^{*}\left(\boldsymbol{\kappa}_{2}\right) F_{1}\left(\boldsymbol{\kappa}_{1}, \boldsymbol{\kappa}_{2}\right) \delta\left(\omega+\left(\sigma_{1}+\sigma_{2}\right)\right) \\
& +A_{m}\left(\boldsymbol{\kappa}_{1}\right) A_{m}^{*}\left(\boldsymbol{\kappa}_{2}\right) F_{2}\left(\boldsymbol{\kappa}_{1}, \boldsymbol{\kappa}_{2}\right) \delta\left(\omega-\left(\sigma_{1}-\sigma_{2}\right)\right) \\
& \left.+A_{m}^{*}\left(\boldsymbol{\kappa}_{1}\right) A_{m}\left(\boldsymbol{\kappa}_{2}\right) F_{2}\left(\boldsymbol{\kappa}_{1}, \boldsymbol{\kappa}_{2}\right) \delta\left(\omega+\left(\sigma_{1}-\sigma_{2}\right)\right)\right] \\
& \times\left[A_{n}^{*}\left(\boldsymbol{\kappa}_{3}\right) A_{n}^{*}\left(\boldsymbol{\kappa}_{4}\right) F_{1}\left(\boldsymbol{\kappa}_{3}, \boldsymbol{\kappa}_{4}\right) \delta\left(\omega-\left(\sigma_{3}+\sigma_{4}\right)\right)\right. \\
& +A_{n}\left(\boldsymbol{\kappa}_{3}\right) A_{n}\left(\boldsymbol{\kappa}_{4}\right) F_{1}\left(\boldsymbol{\kappa}_{3}, \boldsymbol{\kappa}_{4}\right) \delta\left(\omega+\left(\sigma_{3}+\sigma_{4}\right)\right) \\
& +A_{n}^{*}\left(\boldsymbol{\kappa}_{3}\right) A_{n}\left(\boldsymbol{\kappa}_{4}\right) F_{2}\left(\boldsymbol{\kappa}_{3}, \boldsymbol{\kappa}_{4}\right) \delta\left(\omega-\left(\sigma_{3}-\sigma_{4}\right)\right)
\end{aligned}
$$




$$
\begin{aligned}
& \left.+A_{n}\left(\boldsymbol{\kappa}_{3}\right) A_{n}^{*}\left(\boldsymbol{\kappa}_{4}\right) F_{2}\left(\boldsymbol{\kappa}_{3}, \boldsymbol{\kappa}_{4}\right) \delta\left(\omega+\left(\sigma_{3}-\sigma_{4}\right)\right)\right]> \\
& \times e^{-\left(\kappa_{1}+\kappa_{2}\right) z_{s}} e^{-\left(\kappa_{3}+\kappa_{4}\right) z_{s}^{\prime}} d^{2} \kappa_{1} d^{2} \kappa_{2} d^{2} \kappa_{3} d^{2} \kappa_{4} .
\end{aligned}
$$

Since the wave height spectra $A_{m}$ and $A_{n}$ are uncorrelated zero-mean Gaussian random variables as discussed in Sec. 4.2, we can substitute Eq. (4.6) into Eq. (4.32) and integrate over $\kappa_{3}$ and $\kappa_{4}$ to obtain

$$
\begin{aligned}
<B_{m}\left(\mathbf{k}, z_{s}\right) B_{n}^{*}\left(\mathbf{k}^{\prime}, z_{s}^{\prime}\right)>= & \frac{\rho^{2} L_{x}^{2} L_{y}^{2}}{32} \iint_{-\infty}^{\infty} \iint_{\Omega} \sigma_{1}^{2} \sigma_{2}^{2} S_{m n}\left(\boldsymbol{\kappa}_{1}\right) S_{m n}\left(\boldsymbol{\kappa}_{2}\right) \\
& \times\left[F_{1}^{2}\left(\boldsymbol{\kappa}_{1}, \boldsymbol{\kappa}_{2}\right) \delta\left(\omega-\sigma^{+}\right) \delta\left(\omega^{\prime}-\sigma_{1}^{+}\right)\right. \\
& +F_{1}^{2}\left(\boldsymbol{\kappa}_{1}, \kappa_{2}\right) \delta\left(\omega+\sigma^{+}\right) \delta\left(\omega^{\prime}+\sigma^{+}\right) \\
& +F_{2}^{2}\left(\kappa_{1}, \kappa_{2}\right) \delta\left(\omega-\sigma^{-}\right) \delta\left(\omega^{\prime}-\sigma^{-}\right) \\
& +F_{2}^{2}\left(\kappa_{1}, \kappa_{2}\right) \delta\left(\omega-\sigma^{-}\right) \delta\left(\omega^{\prime}+\sigma^{-}\right) \\
& +F_{2}^{2}\left(\kappa_{1}, \kappa_{2}\right) \delta\left(\omega+\sigma^{-}\right) \delta\left(\omega^{\prime}-\sigma^{-}\right) \\
& \left.+F_{2}^{2}\left(\kappa_{1}, \kappa_{2}\right) \delta\left(\omega+\sigma^{-}\right) \delta\left(\omega^{\prime}+\sigma^{-}\right)\right] \\
& \times e^{-\left(\kappa_{1}+\kappa_{2}\right) z_{s}} e^{-\left(\kappa_{1}+\kappa_{2}\right) z_{s}^{\prime}} d^{2} \kappa_{1} d^{2} \kappa_{2} .
\end{aligned}
$$

Then by integrating $\kappa_{2}$ in Eq. (4.33) over the integration region $\Omega$, corresponding to $\left|\boldsymbol{\kappa}^{+}\right| \leq \frac{\omega}{c}$ and $\left|\boldsymbol{\kappa}^{-}\right| \leq \frac{\omega}{c}$, we find

$$
\begin{aligned}
<B_{m}\left(k, z_{s}\right) B_{n}^{*}\left(k^{\prime}, z_{s}\right)>= & 2 \rho^{2} \pi L_{x}^{2} L_{y}^{2} k^{2} \iint_{-\infty}^{\infty} \sigma_{1}^{4} \kappa_{1}^{4} \\
& \times\left[S_{m n}\left(\kappa_{1}\right) S_{m n}\left(-\kappa_{1}\right) \delta\left(\omega-2 \sigma_{1}\right) \delta\left(\omega^{\prime}-2 \sigma_{1}\right)\right. \\
& +S_{m n}\left(\kappa_{1}\right) S_{m n}\left(-\kappa_{1}\right) \delta\left(\omega+2 \sigma_{1}\right) \delta\left(\omega^{\prime}+2 \sigma_{1}\right) \\
& \left.+4 S_{m n}\left(\kappa_{1}\right) S_{m n}\left(\kappa_{1}\right) \delta(\omega) \delta\left(\omega^{\prime}\right)\right] \\
& \times e^{-2 \kappa_{1}\left(z_{s}+z_{s}^{\prime}\right)} d^{2} \kappa_{1} .
\end{aligned}
$$


Since $\frac{\omega}{c}<<$ this integration leads to the approximations $\kappa_{1} \approx-\kappa_{2}$ in the sum terms and $\kappa_{1} \approx \kappa_{2}$ in the difference terms.

The term $4 S_{m n}\left(\kappa_{1}\right) S_{m n}\left(\kappa_{1}\right) \delta(\omega) \delta\left(\omega^{\prime}\right)$ of Eq. (4.34) leads to a constant $\left(\omega=\omega^{\prime}=\right.$ 0 ) value in the power spectral density that is irrelevant. Also, for the remainder of the paper, we suppress the subscripts of $\kappa_{1}$ and $\sigma_{1}$ to simplify notation.

The wave height cross spectral density $S_{m n}(\kappa)$ can be converted to the frequencyangle $(\sigma-\theta)$ domain $S_{m n}(\sigma, \theta)$ by evaluating the Jacobian $\frac{\partial \kappa}{\partial \sigma} \kappa$ where $\sigma^{2}=g \kappa=g|\kappa|$ [127]. This yields the relation $S_{m n}(\sigma, \theta)=\frac{\partial \kappa}{\partial \sigma} \kappa S_{m n}(\kappa)=\frac{2 \sigma^{3}}{g^{2}} S_{m n}(\kappa)$. Making this substitution in Eq. (4.34) and integrating over $\sigma$ yields,

$$
\begin{aligned}
<B_{m}\left(k, z_{s}\right) B_{n}^{*}\left(k^{\prime}, z_{s}^{\prime}\right)>\approx & \frac{\rho^{2} \pi L_{x}^{2} L_{y}^{2} k^{2} \omega^{9}}{512 g^{2}} \delta\left(\omega^{\prime}-\omega\right) \\
& \times e^{\frac{-\omega^{2} z_{s}}{2 g}\left(z_{s}+z_{s}^{\prime}\right)} \int_{0}^{2 \pi} S_{m n}\left(\frac{\omega}{2}, \theta\right) S_{m n}\left(\frac{\omega}{2}, \theta+\pi\right) d \theta(4 .
\end{aligned}
$$

Note that the delta functions in Eq. (4.34) introduce a frequency doubling effect in Eq. (4.35) where the frequency $\omega$ of the acoustic wave is twice the frequency $\sigma$ of the surface gravity wave.

Substituting Eq. (4.35) into Eq. (4.19) and integrating over $\omega^{\prime}$ leads to the power spectral density of the pressure field

$$
\begin{aligned}
S_{P_{m, n}}\left(\mathbf{r}_{r}, \omega\right)= & \frac{\rho^{2} \pi L_{x}^{2} L_{y}^{2} k^{2} \omega^{9}}{512 g^{2}} \int_{0}^{\lambda_{g}} \int_{0}^{\lambda_{g}} d z_{s} d z_{s}^{\prime} \\
& \times g\left(\mathbf{r}_{r}, \mathbf{r}_{m}, w\right) g^{*}\left(\mathbf{r}_{r}, \mathbf{r}_{n}, w\right) e^{\frac{-\omega^{2} z_{s}}{2 g}} e^{\frac{-\omega^{2} z_{s}^{\prime}}{2 g}} \\
& \times \int_{0}^{2 \pi} S_{m n}\left(\frac{\omega}{2}, \theta\right) S_{m n}\left(\frac{\omega}{2}, \theta+\pi\right) d \theta
\end{aligned}
$$




$$
\begin{aligned}
= & \int_{0}^{\lambda_{g}} \int_{0}^{\lambda_{g}} d z_{s} d z_{s}^{\prime} g\left(\mathbf{r}_{r}, \mathbf{r}_{m}, w\right) g^{*}\left(\mathbf{r}_{r}, \mathbf{r}_{n}, w\right) \\
& \times S_{B_{m n}}\left(\omega, z_{s}\right),
\end{aligned}
$$

where we define the 'microseismic source cross-spectral density' as

$$
\begin{aligned}
S_{B_{m n}}\left(\omega, z_{s}\right)= & \int_{-\infty}^{\infty}<B_{m}\left(k, z_{s}\right) B_{n}^{*}\left(k^{\prime}, z_{s}^{\prime}\right)>d \omega^{\prime} \\
= & \frac{\rho^{2} \pi L_{x}^{2} L_{y}^{2} k^{2} \omega^{9}}{512 g^{2}} e^{\frac{-\omega^{2} z_{s}}{2 g}} e^{\frac{-\omega^{2} z_{s}^{\prime}}{2 g}} \\
& \times \int_{0}^{2 \pi} S_{m n}\left(\frac{\omega}{2}, \theta\right) S_{m n}\left(\frac{\omega}{2}, \theta+\pi\right) d \theta .
\end{aligned}
$$

Summing the contribution from all finite volumes $V_{m}$ gives us the total received power spectral density

$$
\begin{aligned}
S_{P}\left(\mathbf{r}_{r}, \omega\right) \approx & \frac{\rho^{2} \pi L_{x}^{2} L_{y}^{2} k^{2} \omega^{9}}{512 g^{2}} \sum_{m, n} \int_{0}^{\lambda_{g}} \int_{0}^{\lambda_{g}} d z_{s} d z_{s}^{\prime} \\
& \times g\left(\mathbf{r}_{r}, \mathbf{r}_{m}, w\right) g^{*}\left(\mathbf{r}_{r}, \mathbf{r}_{n}, w\right) e^{\frac{-\omega^{2} z_{s}}{2 g}} e^{\frac{-\omega^{2} z_{s}^{\prime}}{2 g}} \\
& \times \int_{0}^{2 \pi} S_{m n}\left(\frac{\omega}{2}, \theta\right) S_{m n}\left(\frac{\omega}{2}, \theta+\pi\right) d \theta \\
\approx & \sum_{m, n} \int_{0}^{\lambda_{g}} \int_{0}^{\lambda_{g}} d z_{s} d z_{s}^{\prime} g\left(\mathbf{r}_{r}, \mathbf{r}_{m}, w\right) g^{*}\left(\mathbf{r}_{r}, \mathbf{r}_{n}, w\right) \\
& \times S_{B_{m n}}\left(\omega, z_{s}\right) .
\end{aligned}
$$

Equation (4.38) provides an analytic expression for microseisms generated by inhomogeneous ocean surface wave fields, as opposed to previous formulations where spatial homogeneity is assumed over infinite $[51,16,54,77,62]$ or very large $[78,63,64]$ 
surface wave areas. Also the Green functions $g\left(\mathbf{r}_{\mathbf{r}}, \mathbf{r}_{\mathbf{m}}, w\right)$ and $g^{*}\left(\mathbf{r}_{\mathbf{r}}, \mathbf{r}_{\mathbf{n}}, w\right)$ in Eq. (4.38) may be calculated for any arbitrary range-dependent or range-independent ocean waveguide using standard propagation models. Later we will use a formulation for range-dependent Rayleigh wave propagation to calculate the Green functions and model microseismic propagation in a typical North Atlantic waveguide environment.

While Eq. (4.38) is applicable to inhomogeneous surface wave fields and arbitrary ocean waveguides, it can also be applied to more simple homogeneous surface wave fields and Green functions. In fact, in App. E we find that, given an infinite halfspace and assuming the surface wave spectrum is range-independent $\left(S_{m n}(\omega / 2, \theta)=\right.$ $S(\omega / 2, \theta))$, the power spectral density of the pressure field in Eq. (4.38) simplifies to the results derived by Hughes [54] and by Lloyd [77].

\subsection{Microseismic Source Levels in Hurricane Bon- nie}

In this section we show how Eq. (4.37) can be used to find the microseismic source cross-spectral density generated by the surface wave field in a real hurricane. In these examples we use the wave spectra from hurricane Bonnie. Through this analysis we demonstrate the relationship between the wind speeds, surface wave spectra, and microseismic source spectra in a hurricane.

Beginning on 22 August 1998, hurricane Bonnie traveled along the east coast of the Bahamas, Florida, and South Carolina [3]. During this time microseisms were recorded by seismometers ${ }^{1}$ at the Disney Wilderness Preserve in Florida $\left(28.1^{\circ} \mathrm{N}\right.$, $\left.81.4^{\circ} \mathrm{W}\right)$. Here we model the microseisms generated by the complex surface wave field

\footnotetext{
${ }^{1}$ The IRIS seismometers are part of the Global Seismic Network (GSN) and are installed, maintained and operated by the United States Geological Survey (USGS) Albuquerque Seismological Laboratory (http://asiwww.cr.usgs.gov and http://www.liss.org). The GSN is a cooperative scientific facility operated jointly by the Incorporated Research Institutions for Seismology (IRIS), the USGS, and the National Science Foundation (NSF).
} 
of hurricane Bonnie.

Evaluating the microseismic source cross-spectral density $S_{B_{m n}}$ of Eq. (4.37) requires knowledge of the surface wave height spectrum $A_{m}$. Moon et al [88] calculate the surface wave height spectrum for the wind speeds measured in hurricane Bonnie using the Wave Watch III (WW3) wind-wave modeling program [126]. They also show how their results are in close agreement with aircraft based measurements of surface wave height spectrum by Wright et al [142] and Walsh et al [132].

Figure 4.2(A) shows the surface wind speed in hurricane Bonnie on 24 August 1998. Illustrated is the typical hurricane structure with the high wind speeds of the 'eye wall' surrounding the 'eye' at the hurricane's center. From these wind speeds, WW3 is used to determine the wave height spectra $A_{m}(\kappa)$ as defined in Eq. (4.5) (Fig. 4.2). In Fig. 4.2 we see that hurricanes generate waves that propagate in many different and often opposing directions. For example in Fig. 4.2(c) we see a peak in the surface wave spectrum (in red) with waves traveling North $\left(\left(\kappa_{x}, \kappa_{y}\right) \approx(0.0,0.4)\right)$, West $\left(\left(\kappa_{x}, \kappa_{y}\right) \approx(-0.4,0.0)\right)$, and South $\left(\left(\kappa_{x}, \kappa_{y}\right) \approx(0.0,-0.4)\right)$.

From the wave height spectra calculated using WW3, the microseismic source cross-spectral density $S_{B_{m n}}$ may be calculated from Eq. (4.37). We define the 'microseismic source level' as

$$
L_{B_{m n}}\left(\omega, z_{s}\right)=10 \log \left(\frac{S_{B_{m n}}\left(\omega, z_{s}\right)}{L_{x}^{2} L_{y}^{2}} \frac{1 m^{4}}{1 P a^{2} 1 s}\right)
$$

expressed in $\mathrm{dB}$ re $\mathrm{Pa}^{2} \mathrm{~s} / \mathrm{m}^{4}$ where the somewhat arbitrary length scales $L_{x}$ and $L_{y}$ have been factored out. Figure $4.2(\mathrm{~B})$ shows $L_{B_{m m}}(\omega, 0)$ at $\omega=1.26 \mathrm{rad} / \mathrm{s}(f=0.2$ $\mathrm{Hz}$ ) on 24 August 1998, the day where we found the source level to be highest. We see that the peak of $L_{B_{m m}}$ at location (c) is not at the same location as the maximum wind speed (a). This is because, while location (a) has large wave heights $(\approx 37$ 
$\mathrm{dB}$ re $\left.\mathrm{m}^{2} /\left(\operatorname{rad}^{2} / \mathrm{m}^{2}\right)\right)$, the waves are all propagating primarily to the South without any opposing waves. At location (c), however, there are opposing waves (propagating both North and South) which generate microseisms as expressed in Eq. (4.37). Also the source level is higher in the low-wind-speed eye (b) than at the maximum wind speed location (a) due to the opposition in the surface waves (Fig. 4.2(b)).

The arrows in Fig. 4.2 represent the direction hurricane Bonnie was moving and we see that there is a small peak in the source level well 'behind' the hurricane at location (d) even though the wind speed there is relatively low. This is again due to the opposing waves at this location (Fig. 4.2(d)). This illustrates the complex relationship between wind speed and wave spectra, where the wave spectra is a function not only of wind speed but also of hurricane geometry and translation speed [88], and the complex relationship between wave spectra and microseismic source level, where the level depends on the nonlinear interaction of opposing waves.

\subsection{Received Microseismic Levels in Florida Due to Hurricane Bonnie}

In this section we model the propagation of the microseisms generated by hurricane Bonnie through the North Atlantic waveguide. The model results are then compared with seismic data gathered in Florida.

To determine the microseisms received at the sensor in Florida the Green functions $g\left(\mathbf{r}_{\mathbf{r}}, \mathbf{r}_{\mathbf{m}}, w\right)$ and $g^{*}\left(\mathbf{r}_{\mathbf{r}}, \mathbf{r}_{\mathbf{n}}, w\right)$ of Eq. (4.38) are calculated using the adiabatic Rayleigh wave propagation model derived in App. F. The bathymetry between the hurricane, located over the roughly $5 \mathrm{~km}$ deep Hatteras Abyssal plain on 24 August, and the seismometer, located in Florida is characterized by a gentle upslope. We will model this up-sloping environment with the simplified geometry shown in Fig 4.6. The sound speeds and densities for this model waveguide are based on typical values for 
Table 4.2: Parameters for Hurricane Bonnie on 24 August 1998

\begin{tabular}{|c|c|c|c|c|}
\hline Time & $\begin{array}{l}\text { Hurricane } \\
\text { Lat }\left({ }^{\circ} \mathrm{N}\right)\end{array}$ & $\begin{array}{l}\text { enter Position } \\
\text { Lon }\left({ }^{\circ} \mathrm{W}\right)\end{array}$ & $\begin{array}{l}\text { Range from } \\
\text { Sensor }(\mathrm{km})\end{array}$ & $\begin{array}{c}\text { Ocean Depth at } \\
\text { Hurricane Center }(\mathrm{km})\end{array}$ \\
\hline 0000 & 24.8 & 71.8 & 1028 & 5.1 \\
\hline 0600 & 25.2 & 72.1 & 983 & 5.5 \\
\hline 1200 & 25.6 & 72.4 & 939 & 5.5 \\
\hline 1800 & 26.1 & 72.8 & 881 & 5.2 \\
\hline
\end{tabular}

the deep North Atlantic ocean [83] and bottom [73].

With this environment the Green functions $g\left(\mathbf{r}_{\mathbf{r}}, \mathbf{r}_{\mathbf{m}}, w\right)$ and $g^{*}\left(\mathbf{r}_{\mathbf{r}}, \mathbf{r}_{\mathbf{n}}, w\right)$ are calculated using Eq. (F.23). The sea-floor depth at the center of hurricane Bonnie is given in Table 4.6, as well as the range between the seismometer and the hurricane center. The seismometer in Florida is buried in the ground to a depth of $162 \mathrm{~m}$ as shown in Fig. 4.3.

Given the source cross-spectral density from Eq. (4.37) and Green functions from Eq. (F.23), the power spectral density of the field, from Eq. (4.38), received by the seismometer in Florida is calculated. Figure 4.6 shows the power spectral density of the horizontal velocity (Fig. 4.6(a-d)) and vertical velocity (Fig. 4.6(e-h)) of the earth's crust at the Florida seismometer based on our model (in red) and on measured data (in blue).

The dashed blue lines in Fig. 4.6 represent portions of the measured data that are corrupted by non-hurricane related ambient noise. The level of this ambient noise is determined by measuring the noise levels during the week before and the week after the hurricane passed. The un-corrupted microseism signals are taken to be those that exceed the ambient by at least $3 \mathrm{~dB}$ while anything below that is considered to be corrupted.

The theoretical results and measured data show reasonable agreement with peaks in the spectrum at roughly $f=0.2 \mathrm{~Hz}$ and a peak level between 50 and $60 \mathrm{~dB}$ re 


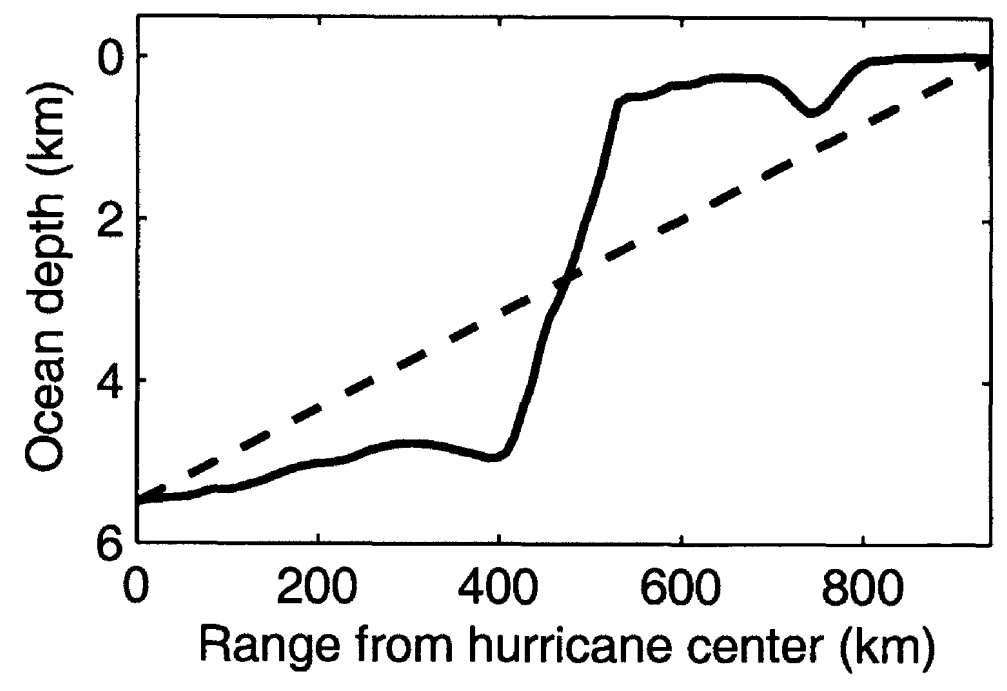

Figure 4-4: The ocean depth (solid line) between hurricane Bonnie and the seismometer in Florida at noon on 24 August. Also shown is the ocean depth for the idealized up-sloping environment used to calculate the Green functions in Section 4.6 (dashed line). The scale of the figure makes the actual slope appear to change rapidly, however, the maximum slope of the ocean floor is roughly $3^{\circ}$. 

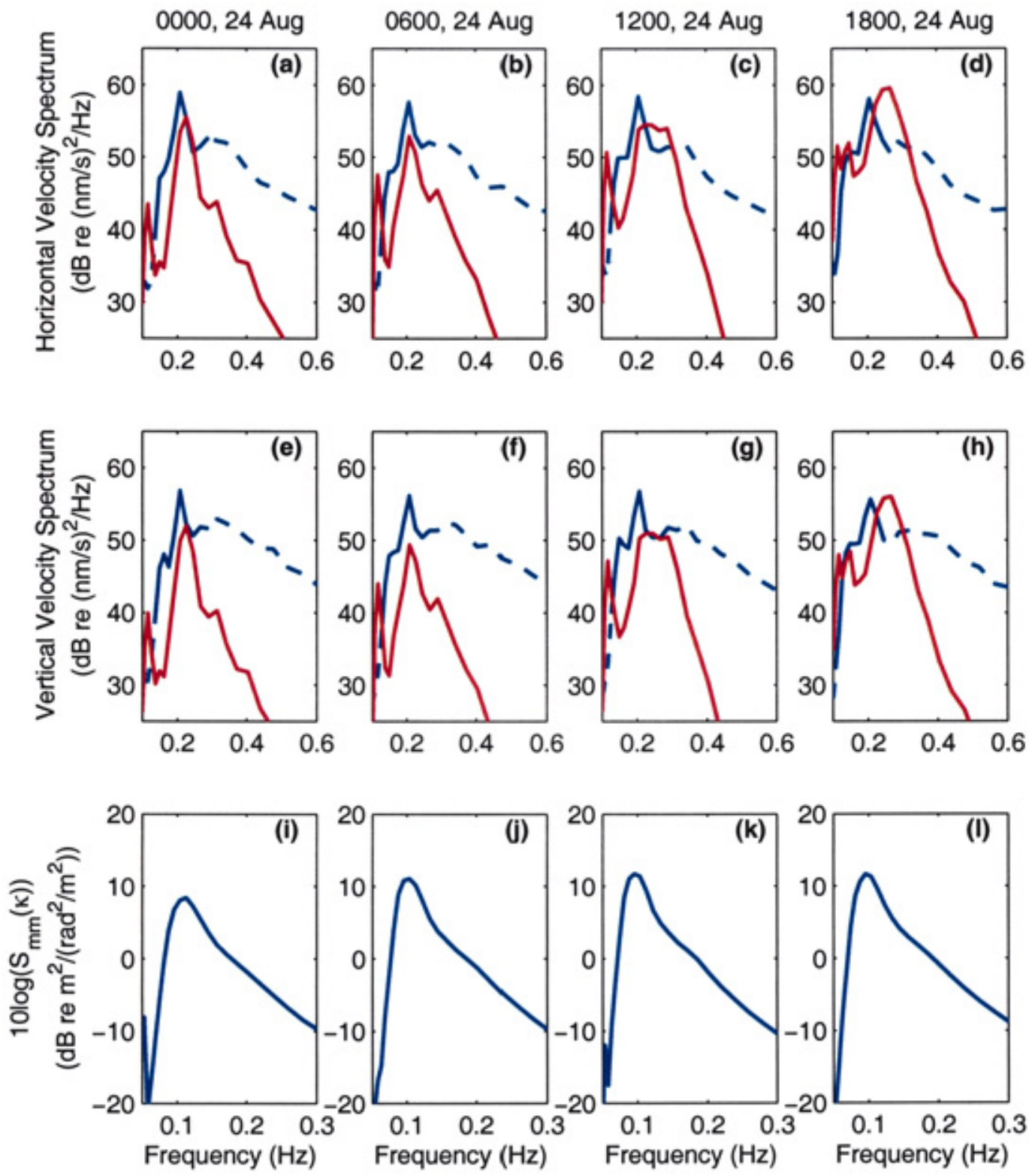

Figure 4-5: Horizontal (a-d) and vertical (e-h) velocity spectra, as a function of seismic frequency $(f=2 \pi \omega)$, modeled (red) and measured (blue) at the seismometer in Florida at four times on 24 August 1998. The dashed blue lines represent portions of the data that where corrupted by non-hurricane related ambient noise. Note that the peak in the spectra is at roughly $f=0.2 \mathrm{~Hz}$. This frequency corresponds to the peak in the wave-height power spectral level at $\kappa=0.04 \mathrm{rad} / \mathrm{m}$ (surface wave frequency $f=2 \pi \sigma=0.1 \mathrm{~Hz}$ ) seen in Fig. 4.2 and in plots (i-1). The surface waveheight power spectral level $\left(10 \log S_{m m}(\kappa) /\left(m^{2} /\left(\mathrm{rad}^{2} / \mathrm{m}^{2}\right)\right)\right)$ in plots (i-l) is taken at the center of the storm and averaged over wave propagation direction. 
$(\mathrm{nm} / \mathrm{s})^{2} / \mathrm{Hz}$. Note that the peak of the wave height spectrum in Fig. 4.2 is at roughly $\kappa=0.04 \mathrm{rad} / \mathrm{m}$ which equates to a surface wave frequency $\sigma=0.63 \mathrm{rad} / \mathrm{s}$. From the frequency doubling effect discussed in Sec 4.4, this gives a peak in the acoustic field at $\omega=1.26 \mathrm{rad} / \mathrm{s}$ or $f=0.2 \mathrm{~Hz}$ which we see in the received field in Fig. 4.6.

\subsection{Conclusions}

Here we present an analytic model, based on the non-linear wave equation, to describe the microseisms generated by a hurricane. This model is ideal for hurricane generated microseisms since it can be used to calculate the acoustic field due to spatially inhomogeneous surface waves. Also, this model may be used in range-dependent waveguide environments as is the case when a hurricane at sea generates microseisms that propagate up the continental margin to a receiver on land. This modeling is useful because microseisms are a primary cause of noise in seismic measurements $[74,107,105]$ that raise the detection threshold for monitoring earthquakes [135] and tsunamis.

Based on the ocean surface directional wave spectrum in hurricane Bonnie[142, 88], we hindcast the microseismic source levels generated by the nonlinear interaction of the ocean surface waves. We then model propagation of the microseismic field from hurricane Bonnie in the North Atlantic to a seismometer in Florida to hindcast the measured signal. We find that these results compare reasonably well with actual seismic measurements. 


\section{Chapter 5}

\section{Conclusion}

We have shown that the wind-generated noise in the $10 \mathrm{~Hz}$ to $10 \mathrm{kHz}$ range received by a single underwater acoustic sensor in a hurricane can be well approximated by sea-surface contributions so local that wind speed and surface source intensity can be taken as nearly constant. Two terms with empirically and analytically determined dependencies may be used to estimate wind speed from measured ambient noise spectral level (1) a universal ambient noise source term and (2) a local waveguide calibration term. At low frequencies, current evidence, including measurement of the underwater noise from hurricane Gert, demonstrate a simple power-law relationship exists between noise intensity and wind speed so that the log of wind speed may be estimated accurately from spectral ambient noise level by linear least square estimation. The power-law relationship and small RMSE between noise intensity and local hurricane wind speed shows that estimates of hurricane wind speed based on underwater noise measurements could be very accurate with an error margin of only $5 \%$. At higher frequencies, a non-linear relationship is expected but we show that it should be possible to make unambiguous low-variance wind speed estimates from broad-band noise measurements. An underwater acoustic sensor could then effectively act as an acoustic anemometer, providing an accurate estimate of a hurricane's wind speeds and 
destructive power. Currently many ocean acoustic systems exist, such as the PMEL sensor used in this work, that could be used for meteorological measurements and additional systems could be deployed from ships, aircraft, or near shore at relatively low cost.

We also develop a new theoretical model for the 0.1 to $1 \mathrm{~Hz}$ microseisms generated by the nonlinear interaction of sea surface waves. This model is suitable for hurricane generated microseisms since it accounts for surface wave fields that change with position and for complex and potentially range-dependent environments. The microseisms generated by hurricane Bonnie and received by a seismometer in Florida is calculated and shown to agree favorably with measured data. This analysis may be useful because microseisms are a primary cause of noise in seismic measurements $[74,107,105]$ of earthquakes[135] and tsunamis. It may also be useful for determining the isotropy of the microseismic field necessary for surface wave tomography [117]. 


\section{Appendix A}

\section{Noise Correlation from Random}

\section{Surface Sources}

Previous models for sea-surface noise[70, 95, 19] contain approximations or parameterizations that are not valid for the rapidly spatially-varying source levels of a hurricane, particularly in the case where the hydrophone is near or under the hurricane. Because of this an alternative expression for calculating the spatial cross-spectral density of the noise field is necessary. The geometry for modeling the spatial cross-spectral density from uncorrelated noise sources at the surface of a stratified ocean waveguide is shown in Fig. 2-2.

The pressure field created by each surface source is given by the solution to the Helmholtz equation

$$
\phi(\mathbf{r}, f)=q\left(\mathbf{r}_{\mathbf{0}}, f\right) g\left(\mathbf{r} \mid \mathbf{r}_{\mathbf{0}}, f\right)
$$

where $\phi(\mathbf{r}, f)$ is the pressure at $\mathbf{r}$ given a source spectral amplitude $q\left(\mathbf{r}_{0}, f\right)$ at $\mathbf{r}_{0}$ and $g\left(\mathbf{r} \mid \mathbf{r}_{\mathbf{0}}, f\right)$ is the waveguide Green function. The total noise field $\phi_{S}(\mathbf{r}, f)$ is the sum of the fields radiated from each source 


$$
\phi_{S}(\mathbf{r}, f)=\sum_{m}^{M} \phi(\mathbf{r}, f)=\sum_{m}^{M} q\left(\mathbf{r}_{\mathbf{m}}, f\right) g\left(\mathbf{r} \mid \mathbf{r}_{\mathbf{m}}, f\right)
$$

The spatial correlation of the total field between two receivers $\mathbf{r}_{\mathbf{1}}$ and $\mathbf{r}_{\mathbf{2}}$ can then be written as

$$
\begin{aligned}
R\left(\mathbf{r}_{1}, \mathbf{r}_{\mathbf{2}}, f^{\prime}, f^{\prime \prime}\right)= & <\phi_{S}\left(\mathbf{r}_{\mathbf{1}}, f^{\prime}\right) \phi_{S}^{*}\left(\mathbf{r}_{\mathbf{2}}, f^{\prime \prime}\right)> \\
= & \sum_{m} \sum_{n}<q\left(\mathbf{r}_{\mathbf{m}}, f^{\prime}\right) q^{*}\left(\mathbf{r}_{\mathbf{n}}^{\prime}, f^{\prime \prime}\right)> \\
& \times g\left(\mathbf{r}_{\mathbf{1}} \mid \mathbf{r}_{\mathbf{m}}, f^{\prime}\right) g^{*}\left(\mathbf{r}_{\mathbf{2}} \mid \mathbf{r}_{\mathbf{n}}, f^{\prime \prime}\right)
\end{aligned}
$$

If the sources have zero mean and are uncorrelated, then $\left\langle q\left(\mathbf{r}_{\mathbf{m}}, f^{\prime}\right) q^{*}\left(\mathbf{r}_{\mathbf{n}}, f^{\prime \prime}\right)\right\rangle=$ $\delta_{m, n}<q\left(\mathbf{r}_{\mathbf{m}}, f^{\prime}\right) q^{*}\left(\mathbf{r}_{\mathbf{n}}, f^{\prime \prime}\right)>$, where $\delta_{m, n}$ is the Kronecker delta function and the correlation simplifies to

$$
\begin{aligned}
R\left(\mathbf{r}_{\mathbf{1}}, \mathbf{r}_{\mathbf{2}}, f^{\prime}, f^{\prime \prime}\right)= & \sum_{m}<q\left(\mathbf{r}_{\mathbf{m}}, f^{\prime}\right) q^{*}\left(\mathbf{r}_{\mathbf{m}}, f^{\prime \prime}\right)> \\
& \times g\left(\mathbf{r}_{\mathbf{1}} \mid \mathbf{r}_{\mathbf{m}}, f^{\prime}\right) g^{*}\left(\mathbf{r}_{\mathbf{2}} \mid \mathbf{r}_{\mathbf{m}}, f^{\prime \prime}\right)
\end{aligned}
$$

Assuming that the source function $q$ and the received field $\phi_{S}$ can be taken to follow a stationary random process in time, at least over the measurement period, then $[92]<\phi_{S}\left(\mathbf{r}_{1}, f\right) \phi_{S}^{*}\left(\mathbf{r}_{2}, f^{\prime}\right)>=C\left(\mathbf{r}_{1}, \mathbf{r}_{2}, f\right) \delta\left(f-f^{\prime}\right)$ and $\left\langle q(f) q^{*}\left(f^{\prime}\right)>=\right.$ $S_{q q}(f) \delta\left(f-f^{\prime}\right)$ where $S_{q q}(f)$ is the power-spectral density of $q$ and

$$
C\left(\mathbf{r}_{\mathbf{1}}, \mathbf{r}_{\mathbf{2}}, f\right)=\sum_{m} S_{q q}\left(\mathbf{r}_{\mathbf{m}}, f\right) g\left(\mathbf{r}_{\mathbf{1}} \mid \mathbf{r}_{\mathbf{m}}, f\right) g^{*}\left(\mathbf{r}_{\mathbf{2}} \mid \mathbf{r}_{\mathbf{m}}, f\right)
$$

is the cross-spectral density of $\phi_{S}\left(\mathbf{r}_{1}\right)$ and $\phi_{S}\left(\mathbf{r}_{2}\right)$. 
For dense source concentrations, this sum can be expressed as an area integral

$$
C\left(\mathbf{r}_{1}, \mathbf{r}_{\mathbf{2}}, f\right)=\int d^{2} \boldsymbol{\rho}_{\mathbf{0}} \frac{S_{q q}\left(\mathbf{r}_{\mathbf{0}}, f\right)}{\Delta A} g\left(\mathbf{r}_{1} \mid \mathbf{r}_{\mathbf{0}}, f\right) g^{*}\left(\mathbf{r}_{\mathbf{2}} \mid \mathbf{r}_{\mathbf{0}}, f\right)
$$

where $\Delta A$ is equal to or greater than the coherence area of the random source distribution and corresponds to the smallest differential area summable.

The variance of the source amplitude equals the integrated power spectral density $<|q(t)|^{2}>=\int S_{q q}(f) d f$. Since this variance is asymptotically approximated by the sample variance $\left\langle|q(t)|^{2}>\approx \frac{1}{T} \int_{0}^{T}|q(t)|^{2} d t\right.$ for large measurement windows $T$ and since Parseval's Theorem has $\frac{1}{T} \int_{0}^{T}|q(t)|^{2} d t=\frac{1}{T} \int_{-\infty}^{\infty}|Q(f)|^{2} d f$, we may deduce that

$$
S_{q q}(f) \approx \frac{1}{T}|Q(f)|^{2}
$$

which relates $S_{q q}$ to practical measurements.

Equation (A.6) is similar to an intermediate expression derived by Perkins, Kuperman, Ingenito, Fialkowski and Glattetre (Eq. (6) of Ref. [95]) derived for a significantly different physical scenario as noted in section 2.3. Equation (A.6) can be used to model the spatial correlation of the noise field for uncorrelated surface generated noise when the source distribution or waveguide is range dependent. When the source distribution and environment are range independent, Eq. (A.6) reduces to

$$
\begin{aligned}
C\left(\mathbf{r}_{1}, \mathbf{r}_{2}, f\right)= & \frac{2 \pi S_{q q}(f)}{\Delta A} \int_{k_{r}} k_{r} d k_{r} \\
& \times g\left(k_{r}, z_{1}, z_{0}\right) g^{*}\left(k_{r}, z_{2}, z_{0}\right) J_{0}\left(k_{r}\left|\boldsymbol{\rho}_{\mathbf{1}}-\boldsymbol{\rho}_{\mathbf{2}}\right|\right)
\end{aligned}
$$

following the Kuperman and Ingenito approach[70] where $g\left(k_{r}, z_{1}, z_{0}\right)$ is the wavenumber transform of $g\left(\mathbf{r}_{\mathbf{1}} \mid \mathbf{r}_{\mathbf{0}}, f\right)$. 


\section{Appendix B}

\section{Asymptotic Variance for Wind Speed Estimates}

The variance of a wind-speed estimate is evaluated numerically in Section 2.4.3 for some specific scenarios. A general analytic expression is derived here for the asymptotic variance of the wind speed estimate for large sample size $\mu$ using Fisher information. For the expected intensity $I$ of an acoustic measurement with a signal-to-noise ratio or time-bandwidth product $\mu$, the inverse Fisher information or Cramer-Rao Lower Bound (CRLB) of a wind speed estimate $\hat{V}$ is given as $[79,80]$

$$
\operatorname{Var}_{\text {asymptotic }}(\hat{V})=\left(\frac{\mu}{I^{2}}\left(\frac{\partial I}{\partial V}\right)^{2}\right)^{-1}
$$

which is the asymptotic variance.[92]

At low frequencies the relationship between intensity and wind speed can be expressed using Eq. (2.22), which, when inserted into Eq. (B.1), yields 


$$
\operatorname{Var}_{\text {asymptotic }}(\hat{V})=\frac{V^{2}}{\mu n^{2}}
$$

At higher frequencies, where attenuation due to bubbles becomes important, the relationship between intensity and wind speed follows Eq. (2.19), substituting Eqs. (2.15), (2.21) and (2.23), so that

$$
\operatorname{Var}_{\text {asymptotic }}(\hat{V})=\frac{V^{2}}{\mu\left(n-\frac{V L}{10 \log (e)} \frac{\partial \alpha}{\partial V}\right)^{2}}
$$

For the attenuation $\alpha(V, f)$ described by Weston[139] in Eq. (2.4), the CRLB becomes

$$
\operatorname{Var}_{\text {asymptotic }}(\hat{V})=\left\{\begin{array}{lll}
\frac{V^{2}}{\mu\left(n-6.46 \times 10^{-7} L V^{3} \sqrt{f}\right)^{2}} & : & f<1.5 k H z \\
\frac{V^{2}}{\mu\left(n-1.69 \times 10^{-8} L V^{3} f\right)^{2}} & : & f>1.5 k H z
\end{array}\right.
$$

For $V=V_{\max }$ where $V_{\max }$ is given in Eq. (2.24), the CRLB goes to infinity indicating that an unbiased estimate of wind speed is not possible for that wind speed and frequency. This problem can be overcome by broadband intensity measurements. For a wind speed estimate given intensity measurements at multiple frequencies, Eq. (B.4) becomes

$$
\begin{aligned}
\operatorname{Var}_{\text {asymptotic }}(\hat{V}) & =\left(\sum_{f} \frac{\mu(f)}{I(f)^{2}}\left(\frac{\partial I(f)}{\partial V}\right)^{2}\right)^{-1} \\
& \approx\left\{\begin{array}{cl}
\left(\sum_{f} \frac{\mu(f)}{V^{2}}\left(n-6.46 \times 10^{-7} L V^{3} \sqrt{f}\right)^{2}\right)^{-1}: f<1.5 k H z \\
\left(\sum_{f} \frac{\mu(f)}{V^{2}}\left(n-1.69 \times 10^{-8} L V^{3} f\right)^{2}\right)^{-1}: & f>1.5 k H z
\end{array}\right.
\end{aligned}
$$


which remains finite and can be made small by increasing the time-bandwidth product $\mu$. 


\section{Appendix C}

\section{Review of "Microseism and}

\section{infrasound generation by cyclones"}

\section{by Bowen, Richard, Mancini,}

\section{Fessatidis and Crooker}

It has long been known that microseisms are generated by the non-linear interaction of occean surface waves [ $78,51,16,54]$. In 2003, however, Bowen, Richard, Mancini, Fessatidis and Crooker [14] published an alternative explanation for the generation of microseisms by sea surface waves.

The theory they proposed is based on the argument that "the sum of the pressure, the kinetic energy per unit volume, and the potential energy per unit volume [of the surface gravity waves] must have the same value at all points in the [water] column, even if any of these variables are varying in time. The immediate consequence of this is that the pressure time dependence at the base of the column of water [the sea floor] is determined by the time dependence of the surface gravity waves at the top of the column, and this effect is independent of depth." In effect they propose that 
the potential field generated by linear surface waves does not decay with depth but rather extends, unattenuated, to the sea floor. This contradicts classic linear wave theory where the linear surface wave field decays exponentially with depth [66]. The purpose of this appendix is to point out the error in their proof in Sec. III that leads to the non-decay of the surface wave field, without commenting on the validity of the rest of the paper

To begin, the authors give the equations for surface wave height in Eq. (14)

$$
\eta(x, y, t)=A \sin (k x-\omega t)
$$

and vertical velocity in Eq. (15)

$$
v(x, y, t)=\omega A \cos (k x-\omega t)
$$

They then seek to determine the pressure at the sea floor using Bernoulli's equation which they write in Eq. (12) as

$$
P_{a}+\rho g \eta(x, y, t)+\frac{1}{2} \rho v(x, y, \eta, t)^{2}=P(x, y,-h)-\rho g h
$$

where $P_{a}$ is atmospheric pressure, $\rho$ is water density, and $z=-h$ is the ocean bottom. Combining these three equations they derive the pressure at the sea floor to be

$$
P(x, y,-h)=P_{a}+\rho g(h+A \sin (k x-\omega t))+\frac{1}{2} \rho \omega^{2} A^{2} \cos (k x-\omega t)^{2}
$$

in Eq. (16), which seems to show that there is a fluctuating pressure on the sea floor 
which does not decay with depth.

The error in this proof is in the use of Bernoulli's equation in Eq. (12). This particular form of Bernoulli's equation is only applicable to steady state flows, not to the time-dependent fluid flow in a surface wave [66]. In other words Eq. (12) was written incorrectly and none of the terms in it should depend on time $t$. Because of this misuse of Bernoulli's equation, the result given in Eq. (16) cannot be justified. 


\section{Appendix D}

\section{Spectral Properties of Ocean Surface Waves}

\section{D.1 Homogeneous Surface Wave Fields}

In Eq. 4.1 we define the complex surface wave height in the form of an inverse Fourier transform such that

$$
\hat{\zeta}(\boldsymbol{\rho}, 0)=\iint_{-\infty}^{\infty} A(\boldsymbol{\kappa}) e^{i \boldsymbol{\kappa} \cdot \boldsymbol{\rho}} d^{2} \boldsymbol{\kappa}
$$

The surface wave height spectra $A(\kappa)$ can then be written as the Fourier transform

$$
A(\boldsymbol{\kappa})=\frac{1}{4 \pi^{2}} \iint_{-\infty}^{\infty} \hat{\zeta}(\boldsymbol{\rho}, 0) e^{-i \boldsymbol{\kappa} \cdot \boldsymbol{\rho}} d^{2} \boldsymbol{\rho}
$$

We assume that surface wave height has zero mean $\left.\left(<\hat{\zeta}_{(} \boldsymbol{\rho}, 0\right)>=0\right)$. Taking the expectation of Eq. (D.2) 


$$
<A(\boldsymbol{\kappa})>=\frac{1}{4 \pi^{2}} \iint_{-\infty}^{\infty}<\hat{\zeta}(\boldsymbol{\rho}, 0)>e^{-i \boldsymbol{\kappa} \cdot \boldsymbol{\rho}} d^{2} \boldsymbol{\rho}
$$

we can see that the wave height spectra must also have zero mean $(<A(\kappa)\rangle=0)$. The second moment $<A(\kappa) A^{*}\left(\boldsymbol{\kappa}^{\prime}\right)>$ can also be derived using Eq. D.2 such that

$$
\begin{aligned}
<A(\boldsymbol{\kappa}) A^{*}\left(\boldsymbol{\kappa}^{\prime}\right)> & =<\frac{1}{4 \pi^{2}} \iint_{-\infty}^{\infty} \hat{\zeta}(\boldsymbol{\rho}, 0) e^{-i \boldsymbol{\kappa} \cdot \boldsymbol{\rho}} d^{2} \boldsymbol{\rho} \frac{1}{4 \pi^{2}} \iint_{-\infty}^{\infty} \hat{\zeta}^{*}\left(\boldsymbol{\rho}^{\prime}, 0\right) e^{i \boldsymbol{\kappa}^{\prime} \cdot \boldsymbol{\rho}^{\prime}} d^{2} \boldsymbol{\rho}^{\prime}> \\
& =\frac{1}{16 \pi^{4}} \iint_{-\infty}^{\infty} \iint_{-\infty}^{\infty}<\hat{\zeta}(\boldsymbol{\rho}, 0) \hat{\zeta}^{*}\left(\boldsymbol{\rho}^{\prime}, 0\right)>e^{-i \boldsymbol{\kappa} \cdot \boldsymbol{\rho}} e^{i \boldsymbol{\kappa}^{\prime} \cdot \boldsymbol{\rho}^{\prime}} d^{2} \boldsymbol{\rho} d^{2} \boldsymbol{\rho}^{\prime}(\mathrm{D} .4)
\end{aligned}
$$

If we define $\rho^{\prime \prime}=\rho-\rho^{\prime}$ we can write this as

$$
\begin{aligned}
<A(\boldsymbol{\kappa}) A^{*}\left(\boldsymbol{\kappa}^{\prime}\right)> & =\frac{1}{16 \pi^{4}} \iint_{-\infty}^{\infty} \iint_{-\infty}^{\infty} R\left(\boldsymbol{\rho}^{\prime \prime}\right) e^{-i \boldsymbol{\kappa} \cdot \boldsymbol{\rho}} e^{i \boldsymbol{\kappa}^{\prime} \cdot\left(\boldsymbol{\rho}-\boldsymbol{\rho}^{\prime \prime}\right)} d^{2} \boldsymbol{\rho} d^{2} \boldsymbol{\rho}^{\prime \prime} \\
& =\frac{1}{16 \pi^{4}} \iint_{-\infty}^{\infty} R\left(\boldsymbol{\rho}^{\prime \prime}\right) e^{-i \boldsymbol{\kappa} \cdot \boldsymbol{\rho}^{\prime \prime}} d^{2} \boldsymbol{\rho}^{\prime \prime} \iint_{-\infty}^{\infty} e^{-i \boldsymbol{\kappa} \cdot \boldsymbol{\rho}} e^{i \boldsymbol{\kappa}} \cdot \boldsymbol{\rho}_{d^{2} \boldsymbol{\rho}} \\
& =S(\boldsymbol{\kappa}) \delta\left(\boldsymbol{\kappa}-\boldsymbol{\kappa}^{\prime}\right)
\end{aligned}
$$

where $R\left(\boldsymbol{\rho}^{\prime \prime}\right)=<\hat{\zeta}(\boldsymbol{\rho}, 0) \hat{\zeta}^{*}\left(\boldsymbol{\rho}^{\prime}, 0\right)>$ is the wave height correlation and the power spectral density $S(\kappa)$ is its Fourier transform. This expression shows that, for homogeneous surface wave fields, the different wavenumber components are uncorrelated.

In Sec. 4.2 we also assume, based on the Central Limit Theorem, that the complex wave height $\left.\hat{\zeta}_{(} \boldsymbol{\rho}, t\right)$ is a Gaussian random variable. Since the spectrum $A(\kappa)$ is the Fourier transform of the complex wave height (Eq. (D.2), it must also be a Gaussian random variable. The fourth moment of a Gaussian random variable can be written in terms of its second moments such that 


$$
\begin{array}{r}
<A\left(\boldsymbol{\kappa}_{1}\right) A\left(\boldsymbol{\kappa}_{2}\right) A^{*}\left(\boldsymbol{\kappa}_{3}\right) A^{*}\left(\boldsymbol{\kappa}_{4}\right)>= \\
\delta\left(\boldsymbol{\kappa}_{1}-\boldsymbol{\kappa}_{3}\right) S\left(\boldsymbol{\kappa}_{1}\right) \delta\left(\boldsymbol{\kappa}_{2}-\boldsymbol{\kappa}_{4}\right) S\left(\boldsymbol{\kappa}_{2}\right) \\
+\delta\left(\boldsymbol{\kappa}_{1}-\boldsymbol{\kappa}_{4}\right) S\left(\boldsymbol{\kappa}_{1}\right) \delta\left(\boldsymbol{\kappa}_{2}-\boldsymbol{\kappa}_{3}\right) S\left(\boldsymbol{\kappa}_{2}\right)
\end{array}
$$

We can also examine the cross correlation between two surface wave processes $\hat{\zeta}_{m}$ and $\hat{\zeta}_{n}$ where

$$
\hat{\zeta}_{m}(\boldsymbol{\rho}, 0)=\iint_{-\infty}^{\infty} A_{m}(\boldsymbol{\kappa}) e^{i \boldsymbol{\kappa} \cdot \boldsymbol{\rho}} d^{2} \boldsymbol{\kappa}
$$

end

$$
\hat{\zeta}_{n}(\boldsymbol{\rho}, 0)=\iint_{-\infty}^{\infty} A_{n}(\boldsymbol{\kappa}) e^{i \boldsymbol{\kappa} \cdot \boldsymbol{\rho}} d^{2} \boldsymbol{\kappa}
$$

The surface wave height spectra can then be written as the Fourier transform

$$
A_{m}(\boldsymbol{\kappa})=\frac{1}{4 \pi^{2}} \iint_{-\infty}^{\infty} \hat{\zeta}_{m}(\boldsymbol{\rho}, 0) e^{-i \boldsymbol{\kappa} \cdot \boldsymbol{\rho}} d^{2} \boldsymbol{\rho}
$$

and

$$
A_{n}(\boldsymbol{\kappa})=\frac{1}{4 \pi^{2}} \iint_{-\infty}^{\infty} \hat{\zeta}_{n}(\boldsymbol{\rho}, 0) e^{-i \boldsymbol{\kappa} \cdot \boldsymbol{\rho}} d^{2} \boldsymbol{\rho}
$$

Again we assume that surface wave heights and the wave height spectra have zero 
mean so that the second moment $<A_{m}(\kappa) A_{n}^{*}\left(\boldsymbol{\kappa}^{\prime}\right)>$ can be written as

$$
\begin{aligned}
<A_{m}(\boldsymbol{\kappa}) A_{n}^{*}\left(\boldsymbol{\kappa}^{\prime}\right)> & =<\frac{1}{4 \pi^{2}} \iint_{-\infty}^{\infty} \hat{\zeta}_{m}(\boldsymbol{\rho}, 0) e^{-i \boldsymbol{\kappa} \cdot \boldsymbol{\rho}} d^{2} \boldsymbol{\rho} \frac{1}{4 \pi^{2}} \iint_{-\infty}^{\infty} \hat{\zeta}_{n}^{*}\left(\boldsymbol{\rho}^{\prime}, 0\right) e^{i \boldsymbol{\kappa}^{\prime} \cdot \boldsymbol{\rho}^{\prime}} d^{2} \boldsymbol{\rho}^{\prime}> \\
& =\frac{1}{16 \pi^{4}} \iint_{-\infty}^{\infty} \iint_{-\infty}^{\infty}<\hat{\zeta}_{m}(\boldsymbol{\rho}, 0) \hat{\zeta}_{n}^{*}\left(\boldsymbol{\rho}^{\prime}, 0\right)>e^{-i \boldsymbol{\kappa} \cdot \boldsymbol{\rho}} e^{i \boldsymbol{\kappa}^{\prime} \cdot \boldsymbol{\rho}^{\prime}} d^{2} \boldsymbol{\rho} d^{2} \boldsymbol{\rho}^{\prime} \\
& =\frac{1}{16 \pi^{4}} \iint_{-\infty}^{\infty} \iint_{-\infty}^{\infty} R_{m n}\left(\boldsymbol{\rho}^{\prime \prime}\right) e^{-i \boldsymbol{\kappa} \cdot \boldsymbol{\rho}} e^{i \boldsymbol{\kappa}^{\prime} \cdot\left(\boldsymbol{\rho}-\boldsymbol{\rho}^{\prime \prime}\right)} d^{2} \boldsymbol{\rho} d^{2} \boldsymbol{\rho}^{\prime \prime} \\
& =\frac{1}{16 \pi^{4}} \iint_{-\infty}^{\infty} R_{m n}\left(\boldsymbol{\rho}^{\prime \prime}\right) e^{-i \boldsymbol{\kappa} \cdot \boldsymbol{\rho}^{\prime \prime}} d^{2} \boldsymbol{\rho}^{\prime \prime} \iint_{-\infty}^{\infty} e^{-i \boldsymbol{\kappa} \cdot \boldsymbol{\rho}} e^{i \boldsymbol{\kappa}^{\prime} \cdot \boldsymbol{\rho}} d^{2} \boldsymbol{\rho} \\
& =S_{m n}(\boldsymbol{\kappa}) \delta\left(\boldsymbol{\kappa}-\boldsymbol{\kappa}^{\prime}\right)
\end{aligned}
$$

where $R_{m n}\left(\boldsymbol{\rho}^{\prime \prime}\right)=<\hat{\zeta}_{m}(\boldsymbol{\rho}, 0) \hat{\zeta}_{n}^{*}\left(\boldsymbol{\rho}^{\prime}, 0\right)>$ is the wave height cross correlation and the cross spectral density $S_{m n}(\kappa)$ is its Fourier transform.

Again we assume that the spectra $A_{m}(\kappa)$ and $A_{n}(\kappa)$ are Gaussian random variables so that the fourth moment can be written as

$$
\begin{array}{r}
<A_{m}\left(\boldsymbol{\kappa}_{1}\right) A_{m}\left(\boldsymbol{\kappa}_{2}\right) A_{n}^{*}\left(\boldsymbol{\kappa}_{3}\right) A_{n}^{*}\left(\boldsymbol{\kappa}_{4}\right)>= \\
\delta\left(\boldsymbol{\kappa}_{1}-\boldsymbol{\kappa}_{3}\right) S_{m n}\left(\boldsymbol{\kappa}_{1}\right) \delta\left(\boldsymbol{\kappa}_{2}-\boldsymbol{\kappa}_{4}\right) S_{m n}\left(\boldsymbol{\kappa}_{2}\right) \\
+\delta\left(\boldsymbol{\kappa}_{1}-\boldsymbol{\kappa}_{4}\right) S_{m n}\left(\boldsymbol{\kappa}_{1}\right) \delta\left(\boldsymbol{\kappa}_{2}-\boldsymbol{\kappa}_{3}\right) S_{m n}\left(\boldsymbol{\kappa}_{2}\right) .
\end{array}
$$

\section{D.2 Inhomogeneous Surface Wave Fields}

Now we consider the case where the surface wave height is defined over a finite area. The complex surface wave height in a finite region centered at the origin can be written as 


$$
\hat{\zeta}_{w}(\boldsymbol{\rho}, 0)=\hat{\zeta}(\boldsymbol{\rho}, t) w(\boldsymbol{\rho})
$$

where the window function $w$ is unity for $-L_{x} / 2<x<L_{x} / 2$ and $-L_{y} / 2<y<L_{y} / 2$ and zero elsewhere. Taking the Fourier transform (Eq. (D.2)) of both sides of Eq. (D.13) yields

$$
A_{w}(\boldsymbol{\kappa})=A(\boldsymbol{\kappa}) * W(\boldsymbol{\kappa})=\iint_{-\infty}^{\infty} A\left(\boldsymbol{\kappa}^{\prime}\right) W_{0}\left(\boldsymbol{\kappa}-\boldsymbol{\kappa}^{\prime}\right) d^{2} \boldsymbol{\kappa}^{\prime}
$$

The second moment of $A_{w}$ can then be written as

$$
\begin{aligned}
<A_{w}(\boldsymbol{\kappa}) A_{w}^{*}\left(\boldsymbol{\kappa}^{\prime \prime}\right)>= & \iint_{-\infty}^{\infty} W\left(\boldsymbol{\kappa}-\boldsymbol{\kappa}^{\prime}\right) d^{2} \boldsymbol{\kappa}^{\prime} \iint_{-\infty}^{\infty} W^{*}\left(\boldsymbol{\kappa}^{\prime \prime}-\boldsymbol{\kappa}^{\prime \prime \prime}\right) d^{2} \boldsymbol{\kappa}^{\prime \prime \prime} \\
& \times<A\left(\boldsymbol{\kappa}^{\prime}\right) A^{*}\left(\boldsymbol{\kappa}^{\prime \prime \prime}\right)>
\end{aligned}
$$

which, from Eq. (D.5) becomes

$$
\begin{aligned}
<A_{w}(\boldsymbol{\kappa}) A_{w}^{*}\left(\boldsymbol{\kappa}^{\prime \prime}\right)> & =\iint_{-\infty}^{\infty} W\left(\boldsymbol{\kappa}-\boldsymbol{\kappa}^{\prime}\right) d^{2} \boldsymbol{\kappa}^{\prime} \iint_{-\infty}^{\infty} W^{*}\left(\boldsymbol{\kappa}^{\prime \prime}-\boldsymbol{\kappa}^{\prime \prime \prime}\right) d^{2} \boldsymbol{\kappa}^{\prime \prime \prime} S\left(\boldsymbol{\kappa}^{\prime}\right) \delta\left(\boldsymbol{\kappa}^{\prime}-\boldsymbol{\kappa}^{\prime \prime \prime}\right) \\
& =\iint_{-\infty}^{\infty} W\left(\boldsymbol{\kappa}-\boldsymbol{\kappa}^{\prime}\right) W^{*}\left(\boldsymbol{\kappa}^{\prime \prime}-\boldsymbol{\kappa}^{\prime}\right) S\left(\boldsymbol{\kappa}^{\prime}\right) d^{2} \boldsymbol{\kappa}^{\prime}
\end{aligned}
$$

As the dimensions $L_{x}$ and $L_{y}$ become much larger than a wavelength $\lambda_{g}$ the functions $W$ begin to approximate delta functions such that 


$$
\begin{aligned}
<A_{w}(\boldsymbol{\kappa}) A_{w}^{*}\left(\boldsymbol{\kappa}^{\prime \prime}\right)> & \approx \int \delta\left(\boldsymbol{\kappa}-\boldsymbol{\kappa}^{\prime}\right) \delta\left(\boldsymbol{\kappa}^{\prime \prime}-\boldsymbol{\kappa}^{\prime}\right) S\left(\boldsymbol{\kappa}^{\prime}\right) d^{2} \boldsymbol{\kappa}^{\prime} \\
& \approx \delta\left(\boldsymbol{\kappa}-\boldsymbol{\kappa}^{\prime \prime}\right) S(\boldsymbol{\kappa})=<A(\boldsymbol{\kappa}) A^{*}\left(\boldsymbol{\kappa}^{\prime \prime}\right)>
\end{aligned}
$$

We can now consider the more complicated case of the cross power spectral density between the surface wave heights in two finte regions $m$ and $n$.

$$
\hat{\zeta}_{w, m}(\boldsymbol{\rho}, 0)=\hat{\zeta}(\boldsymbol{\rho}, 0) w\left(\boldsymbol{\rho}-\boldsymbol{\rho}_{m}\right)
$$

and

$$
\hat{\zeta}_{w, n}(\boldsymbol{\rho}, 0)=\hat{\zeta}(\boldsymbol{\rho}, 0) w\left(\boldsymbol{\rho}-\boldsymbol{\rho}_{n}\right)
$$

which, after taking the Fourier transform, become

$$
A_{w, m}(\boldsymbol{\kappa})=A_{m}(\boldsymbol{\kappa}) *\left(W(\boldsymbol{\kappa}) e^{-i \boldsymbol{\kappa} \cdot \boldsymbol{\rho}_{m}}\right)
$$

and

$$
A_{w, n}(\boldsymbol{\kappa})=A_{n}(\boldsymbol{\kappa}) *\left(W(\boldsymbol{\kappa}) e^{-i \boldsymbol{\kappa} \cdot \boldsymbol{\rho}_{n}}\right)
$$

As before, we can take the second moment which becomes 


$$
\begin{aligned}
\left\langle A_{w, m}(\boldsymbol{\kappa}) A_{w, n}^{*}\left(\boldsymbol{\kappa}^{\prime \prime}\right)>=\right. & \iint_{-\infty}^{\infty} W\left(\boldsymbol{\kappa}-\boldsymbol{\kappa}^{\prime}\right) e^{-i\left(\boldsymbol{\kappa}-\boldsymbol{\kappa}^{\prime}\right) \cdot \boldsymbol{\rho}_{m}} d^{2} \boldsymbol{\kappa}^{\prime} \\
& \times \iint_{-\infty}^{\infty} W^{*}\left(\boldsymbol{\kappa}^{\prime \prime}-\boldsymbol{\kappa}^{\prime \prime \prime}\right) e^{i\left(\boldsymbol{\kappa}^{\prime \prime}-\boldsymbol{\kappa}^{\prime \prime \prime}\right) \cdot \boldsymbol{\rho}_{n}} d^{2} \boldsymbol{\kappa}^{\prime \prime \prime} \\
& \times<A_{m}\left(\boldsymbol{\kappa}^{\prime}\right) A_{n}^{*}\left(\boldsymbol{\kappa}^{\prime \prime \prime}\right)> \\
= & \iint_{-\infty}^{\infty} W\left(\boldsymbol{\kappa}-\boldsymbol{\kappa}^{\prime}\right) e^{-i\left(\boldsymbol{\kappa}-\boldsymbol{\kappa}^{\prime}\right) \cdot \boldsymbol{\rho}_{m}} d^{2} \boldsymbol{\kappa}^{\prime} \\
& \times \iint_{-\infty}^{\infty} W^{*}\left(\boldsymbol{\kappa}^{\prime \prime}-\boldsymbol{\kappa}^{\prime \prime \prime}\right) e^{i\left(\boldsymbol{\kappa}^{\prime \prime}-\boldsymbol{\kappa}^{\prime \prime \prime}\right) \cdot \boldsymbol{\rho}_{n}} d^{2} \boldsymbol{\kappa}^{\prime \prime \prime} \\
& \times S_{m n}\left(\boldsymbol{\kappa}^{\prime}\right) \delta\left(\boldsymbol{\kappa}^{\prime}-\boldsymbol{\kappa}^{\prime \prime \prime}\right) \\
= & \iint_{-\infty}^{\infty} W\left(\boldsymbol{\kappa}-\boldsymbol{\kappa}^{\prime}\right) e^{-i\left(\boldsymbol{\kappa}-\boldsymbol{\kappa}^{\prime}\right) \cdot \boldsymbol{\rho}_{m}} W^{*}\left(\boldsymbol{\kappa}^{\prime \prime}-\boldsymbol{\kappa}^{\prime}\right) e^{i\left(\boldsymbol{\kappa}^{\prime \prime}-\boldsymbol{\kappa}^{\prime}\right) \cdot \boldsymbol{\rho}_{n}} \\
& \times S_{m n}\left(\boldsymbol{\kappa}^{\prime}\right) d^{2} \boldsymbol{\kappa}^{\prime}
\end{aligned}
$$

Again, as the dimensions $L_{x}$ and $L_{y}$ become much larger than a wavelength $\lambda_{g}$ the functions $W$ begin to approximate delta functions such that

$$
<A_{w, m}(\boldsymbol{\kappa}) A_{w, n}^{*}\left(\boldsymbol{\kappa}^{\prime \prime}\right)>\approx e^{-i\left(\boldsymbol{\kappa}-\boldsymbol{\kappa}^{\prime \prime}\right) \cdot\left(\boldsymbol{\rho}_{m}-\boldsymbol{\rho}_{n}\right)} \delta\left(\boldsymbol{\kappa}-\boldsymbol{\kappa}^{\prime \prime}\right) S_{m n}(\boldsymbol{\kappa})
$$

This expression is similar to the previous Eqs. (D.5) and (D.17) with the addition of an exponential phase term. Since the delta function is zero for all $\boldsymbol{\kappa}^{\prime}-\boldsymbol{\kappa}^{\prime} \neq 0$ and since the phase term is unity when $\boldsymbol{\kappa}^{\prime}-\boldsymbol{\kappa}^{\prime}=0$ this expression can be reduced to

$$
<A_{w, m}(\boldsymbol{\kappa}) A_{w, n}^{*}\left(\boldsymbol{\kappa}^{\prime \prime}\right)>\approx \delta\left(\boldsymbol{\kappa}-\boldsymbol{\kappa}^{\prime \prime}\right) S_{m n}(\boldsymbol{\kappa})=<A_{m}(\boldsymbol{\kappa}) A_{n}^{*}\left(\boldsymbol{\kappa}^{\prime \prime}\right)>
$$




\section{Appendix E}

\section{Microseisms in a}

\section{Range-independent half-space}

To compare the expression in Eq. (4.38) with derivations by others we can simplify our solution for the case of a range-independent surface wave field over an infinite ocean half-space. The Green function for a source near the free surface of an infinite ocean half-space can be written as a dipole

$$
g\left(\mathbf{r}_{r}, \mathbf{r}_{m}, \omega\right)=\frac{e^{i k R_{m}}}{2 \pi R_{m}} k z_{s} \cos \left(\beta_{m}\right)
$$

where $R_{m}=\left|\mathbf{r}_{r}-\mathbf{r}_{m}\right|$ is the distance between source and receiver positions $\mathbf{r}_{m}$ and $\mathbf{r}_{r}$ respectively and $\beta_{m}=\operatorname{Atan}\left(\left|\boldsymbol{\rho}_{r}-\boldsymbol{\rho}_{m}\right| /\left|z_{r}-z_{m}\right|\right)$ is the angle from vertical. From Eq. (4.38) we write the power spectral density of the pressure field, substituting Eq. (E.1) as

$$
S_{P}\left(\mathbf{r}_{r}, \omega\right) \approx \frac{\rho^{2} \pi L_{x}^{2} L_{y}^{2} k^{2} \omega^{9}}{512 g^{2}} \int_{0}^{2 \pi} S\left(\frac{\omega}{2}, \theta\right) S\left(\frac{\omega}{2}, \theta+\pi\right) d \theta
$$




$$
\begin{aligned}
& \times \int_{0}^{\lambda_{g}} \int_{0}^{\lambda_{g}} d z_{s} d z_{s}^{\prime} e^{\frac{-\omega^{2} z_{s}}{2 g}} e^{\frac{-\omega^{2} z_{s}^{\prime}}{2 g}} \\
& \times \sum_{m, n} \frac{e^{i k R_{m}}}{2 \pi R_{m}} k z_{s} \cos \left(\beta_{m}\right) \frac{e^{-i k R_{n}}}{2 \pi R_{n}} k z_{s}^{\prime} \cos \left(\beta_{n}\right)
\end{aligned}
$$

For densely spaced $\mathbf{r}_{m}$ and $\mathbf{r}_{n}$ the sums can be approximated as integrals on $\boldsymbol{\rho}_{s}$ and $\rho_{s}^{\prime}$

$$
\begin{aligned}
S_{P}\left(\mathbf{r}_{r}, \omega\right) \approx & \frac{\rho^{2} \pi L_{x}^{2} L_{y}^{2} k^{2} \omega^{9}}{512 g^{2}} \int_{0}^{2 \pi} S\left(\frac{\omega}{2}, \theta\right) S\left(\frac{\omega}{2}, \theta+\pi\right) d \theta \\
& \times \int_{0}^{\lambda_{g}} \int_{0}^{\lambda_{g}} d z_{s} d z_{s}^{\prime} e^{\frac{-\omega^{2} z_{s}}{2 g}} e^{\frac{-\omega^{2} z_{s}^{\prime}}{2 g}} \\
& \times \int_{-\infty}^{\infty} \int_{-\infty}^{\infty} \frac{d^{2} \rho_{s}}{L_{x} L_{y}} \frac{e^{i k R}}{2 \pi R} k z_{s} \cos (\beta) \\
& \times \int_{-\infty}^{\infty} \int_{-\infty}^{\infty} \frac{d^{2} \rho_{s}^{\prime}}{L_{x} L_{y}} \frac{e^{-i k R^{\prime}}}{2 \pi R^{\prime}} k z_{s}^{\prime} \cos \left(\beta^{\prime}\right)
\end{aligned}
$$

For $k z_{r}>>1$ the integral over $\rho_{s}$ can be approximated as $\frac{z_{s}}{L_{x} L_{y}} e^{i k\left(z_{r}-z_{s}\right)+i \pi / 2}$ and the integral over $\rho_{s}^{\prime}$ as $\frac{z_{s}^{\prime}}{L_{x} L_{y}} e^{-i k\left(z_{r}-z_{s}^{\prime}\right)-i \pi / 2}$ so that

$$
\begin{aligned}
S_{P}\left(\mathbf{r}_{r}, \omega\right) \approx & \frac{\rho^{2} \pi k^{2} \omega^{9}}{512 g^{2}} \int_{0}^{2 \pi} S\left(\frac{\omega}{2}, \theta\right) S\left(\frac{\omega}{2}, \theta+\pi\right) d \theta \\
& \times \int_{0}^{\lambda_{g}} \int_{0}^{\lambda_{g}} d z_{s} d z_{s}^{\prime} z_{s} z_{s}^{\prime} e^{\frac{-\omega^{2} z_{s}}{2 g}} e^{\frac{-\omega^{2} z_{s}^{\prime}}{2 g}} e^{i k z_{s}} e^{-i k z_{s}^{\prime}}
\end{aligned}
$$

Integrating over $z_{s}$ then yields

$$
S_{P}\left(\mathbf{r}_{r}, \omega\right) \approx \frac{\rho^{2} \pi k^{2} \omega g^{2}}{32} \int_{0}^{2 \pi} S\left(\frac{\omega}{2}, \theta\right) S\left(\frac{\omega}{2}, \theta+\pi\right) d \theta
$$


since $\frac{\omega^{2}}{2 g}>>k$ at the frequencies considered here. This result is identical to Eq. (33) of Hughes [54] and Eq. (35) of Lloyd [77] where $S(\sigma, \theta)$ equates to $\frac{8 \omega_{f}^{3}}{g^{2}} X\left(k_{1}\right) G(\theta)$ from Hughes and to $\frac{8 \sigma^{3}}{g^{2}} f(\kappa)$ from Lloyd (including the $\frac{1}{2}$ correction of Hughes derivation discussed by Lloyd). 


\section{Appendix F}

\section{Adiabatic Propagation of}

\section{Generalized Rayleigh Waves in a}

\section{Range Dependent Ocean}

\section{Environment}

To determine the microseisms received by a sensor either in the ocean or in the earth's crust, the Green function of Eq. (4.38) must be calculated. It has been shown both theoretically $[78,105]$ and experimentally $[47,75,45,15,11,46]$ that microseisms propagate as Rayleigh waves along the sea floor.

[122] shows how the depth or thickness of the ocean layer affects Rayleigh wave phase speed and how, for large depths or frequencies, there may be multiple propagating Rayleigh modes. $[105,106]$ and $[35]$ later show that the Rayleigh wave field can be expressed as a sum of normal mode contributions. Unlike 'classic' Rayleigh waves which are defined to propagate along a vacuum/elastic boundary, [39] coin the term 'generalized Rayleigh waves' to describe Rayleigh waves that propagate along an elastic boundary, such as at the sea floor, under a finite thickness fluid layer like 
the ocean. The modal expressions of $[105,106]$ and [35] are only applicable, however, to environments where the ocean depth is constant. Varying water depth at the continental margin can affect the Rayleigh wave propagation.

To solve the range-dependent propagation problem, [56] divide the waveguide into range-independent segments, each with its own numerically calculated eigenvalues and eigenfunctions, and apply the adiabatic normal mode approximation to evaluate the seismic and acoustic propagation between segments. The computational nature of their approach, however, does not provide the physical insight given by the analytic results of the earlier range-independent studies $[122,105,106,39,35]$. Here we provide an analytic solution for the range-dependent case where a fluid ocean of varying depth overlays an elastic ocean floor, as opposed to the computational solution method given by Arvello and Überall [56].

We consider the simple case where a monopole source is located in a homogeneous fluid medium overlaying an elastic half space. Unlike previous range-independent studies, here the depth or thickness of the fluid layer may change with range from the source. For the example in this paper we model an up-sloping environment where depth decreases with range, however, other depth profiles may also be considered.

It should be noted that, in addition to modeling microseisms, this range-dependent Rayleigh wave model also has application in other seismic research where a sensor on land measures signals generated by sources at sea. For example undersea earthquakes [124] and underwater explosions, like those monitored under the Comprehensive Nuclear Test Ban Treaty [87, 110], also produce Rayleigh waves on the sea floor. The propagation of these Rayleigh waves is also often measured to infer the geologic characteristics of the ocean floor using surface-wave tomography $[8,103,119]$.

We begin by considering the range-independent form of the normal mode solution for a source at a depth $z_{0}$ in a constant-depth fluid layer overlaying an elastic halfspace [56] 


$$
\begin{aligned}
& \phi_{1}(\rho, z)=\sum_{n} u_{1, n}\left(z_{s}\right) u_{1, n}\left(z_{r}\right) H_{0}^{(1)}\left(k_{n} \rho\right), \\
& \phi_{2}(\rho, z)=\sum_{n} u_{1, n}\left(z_{s}\right) u_{2, n}\left(z_{r}\right) H_{0}^{(1)}\left(k_{n} \rho\right),
\end{aligned}
$$

and

$$
\psi_{2}(\rho, z)=\sum_{n} u_{1, n}\left(z_{s}\right) v_{2, n}\left(z_{r}\right) H_{0}^{(1)}\left(k_{n} \rho\right),
$$

where $\rho=|\rho|$ is the horizontal distance from source to receiver and $z_{r}$ and $z_{s}$ are the receiver and source depth respectivelly. The variable $\phi_{1}$ represents the displacement potential due to compressional waves in the fluid medium, and $\phi_{2}$ and $\psi_{2}$ represent the compression and shear potentials in the solid medium. The variable $k_{n}$ is the horizontal component of the wavenumber and $u_{1, n}, u_{2, n}$ and $v_{2, n}$ are the modal eigenfuctions for each mode $n$. The potentials are related to the horizontal displacement $d_{r}$, vertical displacement $d_{z}$, vertical normal stress $p_{z z}$ and vertical shear stress $p_{z r}$ by $[106,39]$

$$
\begin{aligned}
& d_{r}=\frac{\partial \phi}{\partial r}+\frac{\partial^{2} \psi}{\partial r \partial z} \\
& d_{z}=\frac{\partial \phi}{\partial z}+\frac{\partial^{2} \phi}{\partial z^{2}}+\frac{\omega^{2}}{\beta^{2}} \psi \\
& p_{z z}=\lambda \nabla^{2} \phi+2 \mu \frac{\partial w}{\partial z} \\
& p_{z r}=\mu\left(\frac{\partial q}{\partial z}+\frac{\partial w}{\partial r}\right) .
\end{aligned}
$$


The Lame's constants $\lambda$ and $\mu$ are related to the compressional wave speed in the fluid $v_{1}$ and the compression and shear wave speeds in the solid $\alpha_{2}$ and $\beta_{2}$ by

$$
\begin{aligned}
& v_{1}^{2}=\lambda_{1} / \rho_{1} \\
& \alpha_{2}^{2}=\left(\lambda_{2}+2 \mu_{2}\right) / \rho_{2} \\
& \beta_{2}^{2}=\mu_{2} / \rho_{2},
\end{aligned}
$$

where $\rho_{1}$ and $\rho_{2}$ are the densities in the fluid and elastic media respectively [106, 39].

Pierce [98] has shown that for slowly-varying range-dependent waveguides the modal sums of Eqs. (F.1-F.3) can be written as

$$
\begin{aligned}
\phi_{1}(\rho, z)= & e^{\frac{-i \pi}{4}} \sqrt{\frac{2}{\pi \rho}} \\
& \times \sum_{n} u_{1, n}\left(z_{s}\right) u_{1, n}\left(\rho, z_{r}\right) \frac{e^{-i \int_{0}^{\rho} k_{n}\left(\rho^{\prime}\right) d \rho^{\prime}}}{\sqrt{k_{n}(\rho)}} \\
\phi_{2}(\rho, z)= & e^{\frac{-i \pi}{4}} \sqrt{\frac{2}{\pi \rho}} \\
& \times \sum_{n} u_{1, n}\left(z_{s}\right) u_{2, n}\left(\rho, z_{r}\right) \frac{e^{-i \int_{0}^{\rho} k_{n}\left(\rho^{\prime}\right) d \rho^{\prime}}}{\sqrt{k_{n}(\rho)}}
\end{aligned}
$$

and

$$
\begin{aligned}
\psi_{2}(\rho, z)= & e^{\frac{-i \pi}{4}} \sqrt{\frac{2}{\pi \rho}} \\
& \times \sum_{n} u_{1, n}\left(z_{s}\right) v_{2, n}\left(\rho, z_{r}\right) \frac{e^{-i \int_{0}^{\rho} k_{n}\left(\rho^{\prime}\right) d \rho^{\prime}}}{\sqrt{k_{n}(\rho)}}
\end{aligned}
$$


based on the adiabatic mode approximation. This approximation requires that the change in the waveguide environment as a function of range $\rho$ is negligible over a wavelength scale and that there is no coupling between modes. In these expressions both the modal eigenfunctions $u_{1, n}, u_{2, n}$ and $v_{2, n}$ and the horizontal wavenumber $k_{n}$ are allowed to change with range $\rho$ from the source.

For an iso-speed fluid layer of depth $H(\rho)$ overlaying an elastic half space the general solutions for the mode functions $u_{1, n}, u_{2, n}$ and $v_{2, n}$ can be written in terms of sinusoids and exponentials [106, 35] such that Eqs. (F.6-F.8) become

$$
\begin{aligned}
\phi_{1}(\rho, z)= & e^{\frac{-i \pi}{4}} \sqrt{\frac{2}{\pi \rho}} \\
& \times \sum_{n} \Phi_{1} \sin \left(\xi_{n}(0) z_{s}\right) \sin \left(\xi_{n}(\rho) z_{r}\right) \frac{e^{-i \int_{0}^{\rho} k_{n}\left(\rho^{\prime}\right) d \rho^{\prime}}}{\sqrt{k_{n}(\rho)}} \\
\phi_{2}(\rho, z)= & e^{\frac{-i \pi}{4}} \sqrt{\frac{2}{\pi \rho}} \\
& \times \sum_{n} \Phi_{2} \sin \left(\xi_{n}(0) z_{s}\right) e^{\eta_{n}(\rho) z_{r}} \frac{e^{-i \int_{0}^{\rho} k_{n}\left(\rho^{\prime}\right) d \rho^{\prime}}}{\sqrt{k_{n}(\rho)}}
\end{aligned}
$$

and

$$
\begin{aligned}
\psi_{2}(\rho, z)= & e^{\frac{-i \pi}{4}} \sqrt{\frac{2}{\pi \rho}} \\
& \times \sum_{n} \Psi_{2} \sin \left(\xi_{n}(0) z_{s}\right) e^{\zeta_{n}(\rho) z_{r}} \frac{e^{-i \int_{0}^{\rho} k_{n}\left(\rho^{\prime}\right) d \rho^{\prime}}}{\sqrt{k_{n}(\rho)}}
\end{aligned}
$$

where 


$$
\begin{aligned}
& \xi_{n}(\rho)=\sqrt{\frac{\omega^{2}}{v_{1}^{2}}-k_{n}^{2}(\rho)}=k_{n}(\rho) \sqrt{\frac{c_{n}^{2}(\rho)}{v_{1}^{2}}-1} \\
& \eta_{n}(\rho)=\sqrt{k_{n}^{2}(\rho)-\frac{\omega^{2}}{\alpha_{2}^{2}}}=k_{n}(\rho) \sqrt{1-\frac{c_{n}^{2}(\rho)}{\alpha_{2}^{2}}} \\
& \zeta_{n}(\rho)=\sqrt{k_{n}^{2}(\rho)-\frac{\omega^{2}}{\beta_{2}^{2}}}=k_{n}(\rho) \sqrt{1-\frac{c_{n}^{2}(\rho)}{\beta_{2}^{2}}}
\end{aligned}
$$

represent the vertical components of the wavenumber vector for each mode $n$. The phase speed for each mode is defined as $c_{n}(\rho)=\omega / k_{n}(\rho)$ where $\omega$ is the frequency in radians/sec.

The amplitudes $\Phi_{1}, \Phi_{2}$ and $\Psi_{2}$ of the mode functions in Eqs. (F.9-F.11) must be chosen such that the boundary conditions

$$
\left.\begin{array}{r}
\left(p_{z z}\right)_{f}=\left(p_{z z}\right)_{s} \\
\left(p_{z r}\right)_{s}=0 \\
\left(d_{z}\right)_{f}=\left(d_{z}\right)_{s}
\end{array}\right\} \quad \text { at } \quad z=H(\rho)
$$

are satisfied at the fluid solid interface $z=H(\rho)$. The subscripts $f$ and $s$ indicate that the variable is to be evaluated in the fluid or solid layer respectively. These boundary conditions yield a system of three equations for the unknown amplitudes $\Phi_{1}, \Phi_{2}$ and $\Psi_{2}$ whereby any two of the unknowns may be solved in terms of the third. The third amplitude is then normalized to some convenient value. Later we will choose a normalization such that our solution is consistent with that of Press and Ewing [106] for constant bathymetery $H(\rho)=H$. The solution to this set of equations only exists, however, if the determinant 


$$
\left|\begin{array}{ccc}
-\lambda_{1}\left(\xi_{n}^{2}+k_{n}^{2}\right) \sin \left(\xi_{n} H\right) & \left(-\lambda_{2}\left(\eta_{n}^{2}+k_{n}^{2}\right)+2 \mu_{2} \eta_{n}\right) e^{-\eta_{n} H} & -2 \mu_{2} k_{n}^{2} e^{-\zeta_{n} H} \\
0 & -2 \eta_{n} e^{-\eta_{n} H} & \left(2 \zeta_{n}^{2}+\frac{\omega^{2}}{\beta_{2}^{2}}\right) e^{-\zeta_{n} H} \\
\xi_{n} \cos \left(\xi_{n} H\right) & \eta_{n} e^{-\eta_{n} H} & -k_{n}^{2} e^{-\zeta_{n} H}
\end{array}\right|=(\mathrm{F} .14)
$$

equals zero $[106,39]$ where the dependence of $k_{n}, \xi_{n}, \eta_{n}, \zeta_{n}$ and $H$ on range $\rho$ has been suppressed to simplify notation. Equation (F.14) reduces to the equation

$$
\begin{aligned}
& \frac{\rho_{1}}{\rho_{2}} \frac{\omega^{4}}{\beta_{2}^{4}} \frac{\eta_{n}(\rho)}{\xi_{n}(\rho)} \tan \left(\xi_{n}(\rho) H(\rho)\right) \\
& -\left[4 k_{n}^{2}(\rho) \eta_{n}(\rho) \zeta_{n}(\rho)-\left(2 k_{n}^{2}(\rho)-\frac{\omega^{2}}{\beta_{2}^{2}}\right)^{2}\right]=0
\end{aligned}
$$

where the roots $k_{n}(\rho)$ correspond to the horizontal wavenumbers for each mode $n$. For the case of constant bathymetry $H(\rho)=H$, Eq. (F.15) is identical to those given in earlier works [105, 106, 39], however, for this more general range-dependent case the roots $k_{n}(\rho)$ can now vary with range $\rho$. Unlike a 'classic' Rayleigh wave which has only one root or propagating value, 'generalized' Rayleigh waves may have multiple roots $k_{n}(\rho)$ depending on frequency and fluid layer thickness as shown in Eq. (F.15) for the range-dependent case and as shown by [105] for the range-independent case. Note that Eq. (F.15) has no roots for $k_{n}(\rho)>\omega / v_{1}$.

Given the roots $k_{n}(r)$ from Eq. (F.15), we can express the potentials as

$$
\begin{aligned}
\phi_{1}(\rho, z)= & \frac{2}{H(\rho)} \sqrt{\frac{2 \pi}{\rho}} e^{\frac{-i \pi}{4}} \sum_{n} \Phi_{1}\left(k_{n}(\rho), H(\rho)\right) \\
& \times \sin \left(\xi_{n}(0) z_{s}\right) \sin \left(\xi_{n}(\rho) z\right) \frac{e^{-i \int_{0}^{\rho} k_{n}\left(\rho^{\prime}\right) d \rho^{\prime}}}{\sqrt{k_{n}(\rho)}}
\end{aligned}
$$




$$
\begin{aligned}
\phi_{2}(\rho, z)= & \frac{2}{H(\rho)} \sqrt{\frac{2 \pi}{\rho}} e^{\frac{-i \pi}{4}} \sum_{n} \Phi_{2}\left(k_{n}(\rho), H(\rho)\right) \\
& \times \sin \left(\xi_{n}(0) z_{s}\right) e^{\eta_{n}(\rho) z} \frac{e^{-i \int_{0}^{\rho} k_{n}\left(\rho^{\prime}\right) d \rho^{\prime}}}{\sqrt{k_{n}(\rho)}}
\end{aligned}
$$

and

$$
\begin{aligned}
\psi_{2}(\rho, z)= & 2 \sqrt{\frac{2 \pi}{\rho}} e^{\frac{-i \pi}{4}} \sum_{n} \Psi_{2}\left(k_{n}(\rho), H(\rho)\right) \\
& \times \sin \left(\xi_{n}(0) z_{s}\right) e^{\zeta_{n}(\rho) z} \frac{e^{-i \int_{0}^{\rho} k_{n}\left(\rho^{\prime}\right) d r^{\prime}}}{\sqrt{k_{n}(\rho)}}
\end{aligned}
$$

where the solutions for the modal amplitudes are

$$
\begin{aligned}
& \Phi_{1}\left(k_{n}(r h o), H(\rho)\right)= \\
& -\frac{\frac{\rho_{1}}{\rho_{2}} \frac{c_{n}^{4}(\rho)}{\beta_{2}^{4}} \frac{\eta_{n}(\rho)}{\xi_{n}(\rho)} k_{n}(\rho) H(\rho)}{\sqrt{c_{n}^{2}(\rho) / v_{1}^{2}-1} \cos \left(\xi_{n}(\rho) H(\rho)\right) D\left(k_{n}(\rho), H(\rho)\right)}
\end{aligned}
$$

$$
\begin{aligned}
& \Phi_{2}\left(k_{n}(\rho), H(\rho)\right)= \\
& -\frac{\frac{\rho_{1}}{\rho_{2}} \frac{c_{n}^{2}(\rho)}{\beta_{2}^{2}}\left(2-c_{n}^{2}(\rho) / \beta_{2}^{2}\right) k_{n}(\rho) H(\rho)}{\sqrt{c_{n}^{2}(\rho) / v_{1}^{2}-1} D\left(k_{n}(\rho), H(\rho)\right)}
\end{aligned}
$$

and

$$
\Psi_{1}\left(k_{n}(\rho), H(\rho)\right)=-\frac{2 \frac{\rho_{1}}{\rho_{2}} \frac{c_{n}^{2}(\rho)}{\beta_{2}^{2}} \frac{\eta_{n}(\rho)}{\xi_{n}(\rho)}}{D\left(k_{n}(\rho), H(\rho)\right)}
$$


and where

$$
\begin{aligned}
D\left(k_{n}(\rho), H(\rho)\right)= & \frac{\rho_{1}}{\rho_{2}} \frac{c_{n}^{4}(\rho)}{\beta_{2}^{4}}[ \\
& \frac{\sin \left(\xi_{n}(\rho) H(\rho)\right)}{\sqrt{c_{n}^{2}(\rho) / v_{1}^{2}-1} \sqrt{1-c_{n}^{2}(\rho) / \alpha_{2}^{2}}}\left(1+\frac{1-c_{n}^{2}(\rho) / \alpha_{2}^{2}}{c_{n}^{2}(\rho) / v_{1}^{2}-1}\right) \\
& \left.-\left(\frac{k_{n}(\rho) H(\rho) \sqrt{1-c_{n}^{2}(\rho) / \alpha_{2}^{2}}}{c_{n}^{2}(\rho) / v_{1}^{2}-1} \sec \left(\xi_{n}(\rho) H(\rho)\right)\right)\right] \\
& -4\left[\frac{\sqrt{1-c_{n}^{2}(\rho) / \beta_{2}^{2}}}{\sqrt{1-c_{n}^{2}(\rho) / \alpha_{2}^{2}}}+\frac{\sqrt{1-c_{n}^{2}(\rho) / \alpha_{2}^{2}}}{\sqrt{1-c_{n}^{2}(\rho) / \beta_{2}^{2}}}\right. \\
& +2 \sqrt{1-c_{n}^{2}(\rho) / \alpha_{2}^{2}} \sqrt{1-c_{n}^{2}(\rho) / \beta_{2}^{2}} \\
& \left.-2\left(2-c_{n}^{2}(\rho) / \beta_{2}^{2}\right)\right] \cos \left(\xi_{n}(\rho) H(\rho)\right)
\end{aligned}
$$

Equations (F.16-F.22) provide an analytic model for the propagation of 'generalized' Rayleigh waves in a range dependent environment. Note, however, that the mode amplitudes have been normalized such that $\Phi_{1}\left(k_{n}(\rho), H(\rho)\right), \Phi_{2}\left(k_{n}(\rho), H(\rho)\right)$ and $\Psi_{1}\left(k_{n}(\rho), H(\rho)\right)$ are the same as $\Phi_{1}\left(k_{n}, H\right), \Phi_{2}\left(k_{n}, H\right)$ and $\Psi_{1}\left(k_{n}, H\right)$ of Press and Ewing [106] for the case of constant bathymetry $H(\rho)=H$ (Equation (38) of [106] is missing a factor of 2 which was later corrected in Eq. (4-184) of Ewing, Jardetzky, and Press[39]). The Green function used in this paper can be written as

$$
g\left(\mathbf{r}_{r}, \mathbf{r}_{s}, w\right)=\frac{4 \pi}{f} p_{z z}\left(\left|\boldsymbol{\rho}_{r}, \boldsymbol{\rho}_{s}\right|, z_{r}, z_{s}\right)
$$

where the $\frac{4 \pi}{f}$ accounts for the normalization adopted by Press and Ewing [106]. 


\section{Appendix G}

\section{Review of "Emergence rate of the} time-domain Green's function from the ambient noise cross-correlation function" by Sabra, Roux and

\section{Kuperman}

In the paper "Emergence rate of the time-domain Green's function from the ambient noise cross-correlation function" [113] the authors attempt to show how the waveguide Green function can be determined from measurements of the ambient noise correlation function using the following equation

$$
\frac{d<C_{a b}(\tau)>}{d \tau} \approx-G\left(\mathbf{r}_{a}, \mathbf{r}_{b}, \tau\right)+G\left(\mathbf{r}_{a}, \mathbf{r}_{b},-\tau\right)
$$

Sabra, Roux and Kuperman cite two of their previous papers $[112,111]$ to justify this equation. Reference [111], however, only considers the case of homogeneous noise in 
free space and not the case of typical surface generated noise in an ocean waveguide. Reference [112] does consider surface noise in a waveguide and demonstrates that Eq. (G.1) is not true. Figures 6 and 7 of Ref. [112] show that the right and left hand sides of Eq. (G.1), while exhibiting some similar modal structure, are different. It should be noted that in these figures Sabra, Roux and Kuperman normalized both sides of the equation such that the maximum value is unity thus hiding significant differences in amplitude which we will demonstrate shortly.

To do this we calculate the Green function and noise correlation in a typical shallow water scenario and show that Eq. (G.1) is not valid in that the right hand side and the left hand side are not even approximately equal. To begin the waveguide Green function can be written in the frequency domain as a sum of normal modes

$$
g\left(\mathbf{r}_{a}, \mathbf{r}_{b}, f\right)=\frac{i}{4 \rho} \sum_{m=1}^{\infty} u_{m}\left(z_{a}\right) u_{m}^{*}\left(z_{b}\right) H_{0}^{(1)}\left(k_{m}\left|\boldsymbol{\rho}_{a}-\boldsymbol{\rho}_{b}\right|\right)
$$

where $\mathbf{r}_{a}=\left(\boldsymbol{\rho}_{a}, z_{a}\right)$ and $\mathbf{r}_{b}=\left(\boldsymbol{\rho}_{b}, z_{b}\right)$. For surface generated noise in the ocean, the correlation can be written as

$$
c_{a b}(f)=\frac{\pi q^{2}}{2 \rho^{2} k^{2}} \sum_{m=1}^{\infty} \frac{\left|u_{m}\left(z_{s}\right)\right|^{2} u_{m}\left(z_{a}\right) u_{m}^{*}\left(z_{b}\right) J_{0}\left(k_{m} r\right)}{\alpha_{m} \kappa_{m}}
$$

following the convention of Kuperman and Ingenito [70]. In this formulation $k_{m}=$ $\kappa_{m}+i \alpha_{m}$ and $z_{s}$ is a surface source depth close to $\left(z_{s}<<\lambda\right)$ the ocean surface.

We calculate both the Green function and the noise correlation over the 10 to 2000 $\mathrm{Hz}$ frequency range using mode shapes $u_{m}$ and wavenumbers $k_{m}$ from the KrakenC normal mode code. In this example the waveguide is $110 \mathrm{~m}$ deep isovelocity water layer $\left(\mathrm{c}=1500 \mathrm{~m} / \mathrm{s}, \rho=1000 \mathrm{~kg} / \mathrm{m}^{3}, \alpha=6 \times 10^{-5} \mathrm{~dB} / \lambda\right)$ over a halfspace $(\mathrm{c}=1800$ $\left.\mathrm{m} / \mathrm{s}, \rho=1800 \mathrm{~kg} / \mathrm{m}^{3}, \alpha=1 \mathrm{~dB} / \lambda\right)$. The receivers $a$ and $b$ are at a depth of $50 \mathrm{~m}$ and $20 \mathrm{~km}$ apart. Through Fourier synthesis we can then find the time-domain Green function and correlation. 
With the time-domain Green function and correlation we calculate the right hand side (RHS) and left hand side (LHS) of Eq. (G.1) as shown in Figs. G-1 and G-2. The time series shown in these figures start at $\tau=13.33 \mathrm{sec}$, which is the time required for sound to travel from point $\mathbf{r}_{a}$ to point $\mathbf{r}_{b}$. Figure G-1 compares the RHS and LHS over the first tenth of a second in both log and linear domain. First we notice that while the peaks of both the RHS and LHS occur at the same time they are not equal as suggested by Eq. (G.1). These peaks correspond to the modal arrivals with the lower order modes coming first $(\approx 13.35 \mathrm{sec})$ and the higher order modes coming later. We see, however, that early on the peaks of the RHS are higher but that they decays with time. Figure G-2 shows the comparison over a longer $(2 \mathrm{sec})$ time history and we see that the RHS continues to decay while the LHS remains fairly constant.

From these figures we see that the RHS, which depends on the Green function, 'favors' the low order early arriving modes, while the LHS, which depends on the correlation, favors the higher order later modes. This difference can be explained if we compare Eq. (G.2) for the Green function with Eq. (G.3) for the correlation. Both consist of a sum over modes $u_{m}\left(z_{a}\right)$ and $u_{m}\left(z_{b}\right)$, however, the correlation has additional $\frac{\left|u_{m}\left(z_{s}\right)\right|^{2}}{\alpha_{m} \kappa_{m}}$ term which effectively weights the sum over $m$.

This dependence on $m$ can be simulated using a normal mode code. Figure G-3 for an isovelocity waveguide shows that $\frac{\left|u_{m}\left(z_{s}\right)\right|^{2}}{\alpha_{m} \kappa_{m}}$ varies significantly with $m$ where $z_{s}<<\lambda$. We see that the weighting tends to minimize low order modes in the correlation and accentuates high order modes. This explains why the LHS in Figs. G-1 and G-2 is lower than the RHS for the early arriving low-order modes and higher for the later arriving high-order modes. 

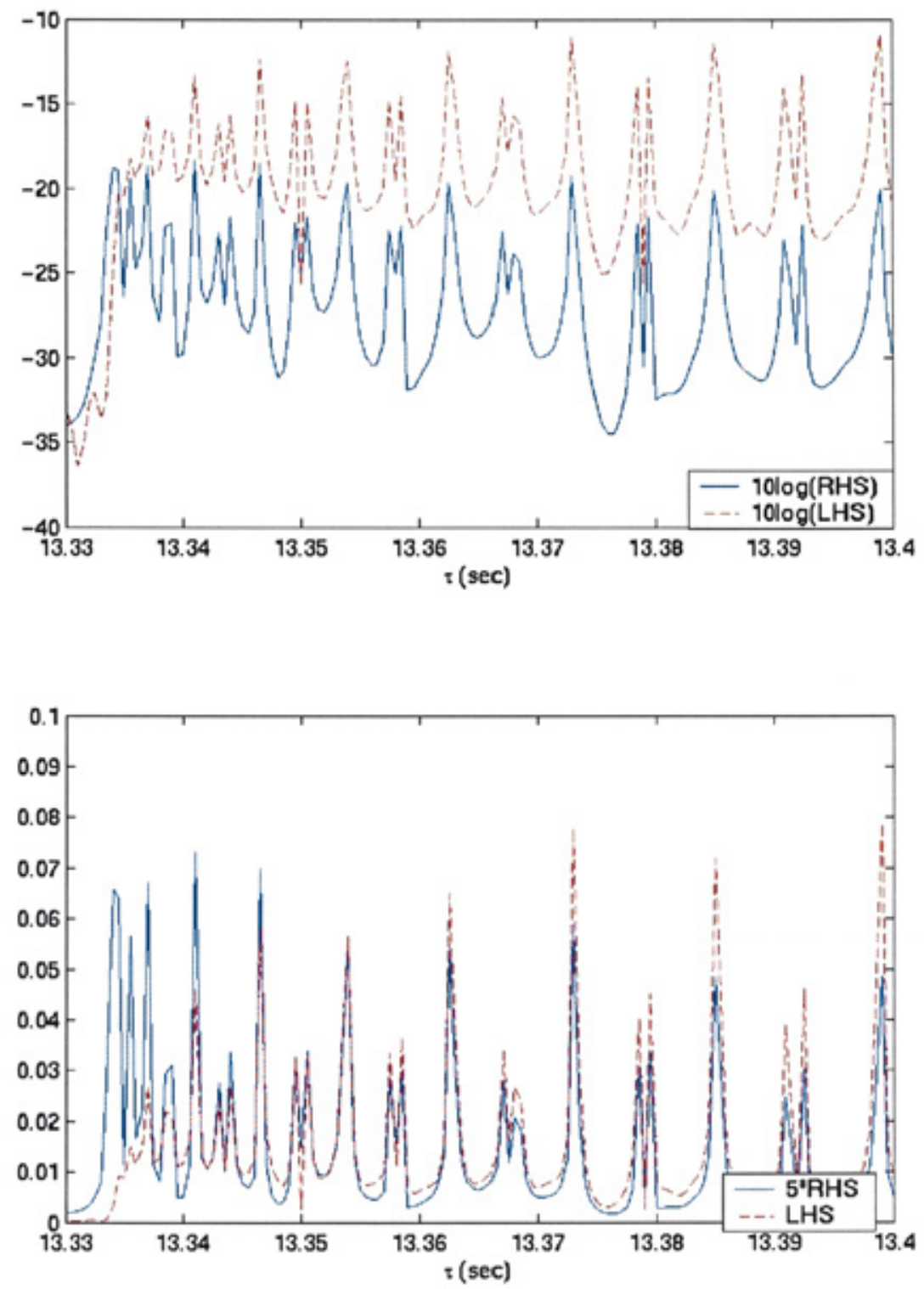

Figure G-1: The RHS and LHS in log (upper plot) and linear (lower plot) domains from 13.33 to 13.4 seconds. We see that the peaks in the curves occur at the same times but that the amplitudes of the peaks differ significantly. We also see that the RHS peaks are higher for the earlier low-order modes while the LHS peaks are higher for the later high-order modes. Note that the RHS in the lower plot has been scaled by a factor of five so that it can be compared against the LHS. 

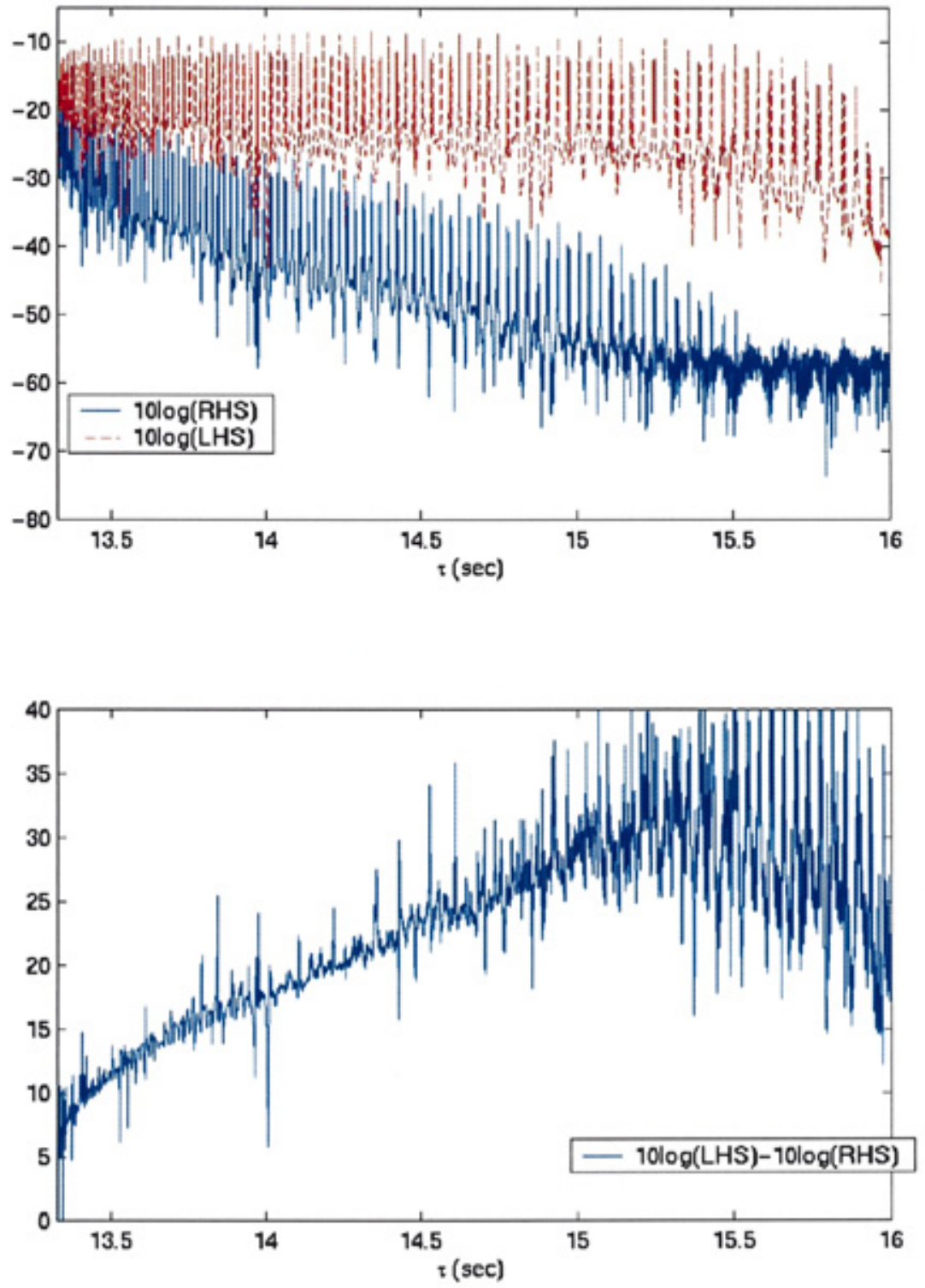

Figure G-2: The RHS and LHS (upper plot) over a longer time duration. We continue to see the general trend where the peaks of the RHS are higher for low-order early arrivals and lower for later high-order arrivals. This can also be seen in the lower plot which shows the difference between the RHS and LHS. 


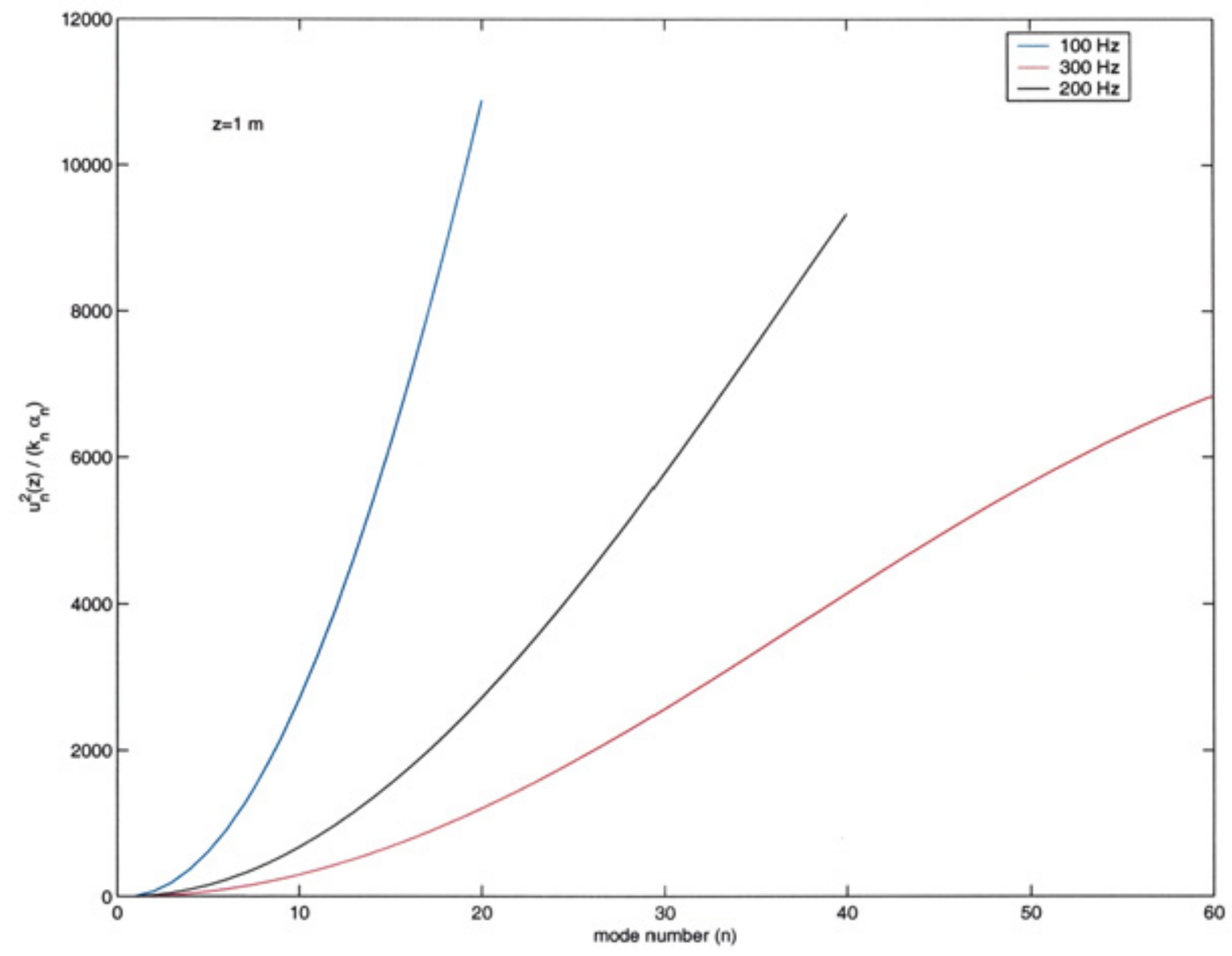

Figure G-3: The weighting term $\frac{\left|u_{m}\left(z_{s}\right)\right|^{2}}{\alpha_{m} \kappa_{m}}$ as a function of mode number $m$ at several frequencies in an isovelocity waveguide. The source depth $z_{s}<<\lambda$ typical of ocean surface noise. 


\section{Bibliography}

[1] Main acoustic clutter experiment, initial report, april 24 - may 24. Technical report.

[2] Office of Public Affairs Air Force Reserve Command. U.S. Air Force fact sheet, WC-130 Hercules. www.af.mil/factsheets/factsheet.asp?fsID=132.

[3] L. A. Avila. Preliminary report, hurricane Bonnie 19-30 August 1998. Technical report, National Hurricane Center, 1998.

[4] L. A. Avila. Tropical cyclone report, hurricane Iris, 4 - 9 October 2001. Technical report, National Hurricane Center, 2001.

[5] S. K. Banerji. Microseisms associated with disturbed weather in the indian seas. Phil. Trans. Roy. Soc. Lon. Ser. A., 229:287-328, 1930.

[6] S. K. Banerji. Theory of microseisms. Proc. Indian Acad. Sci., 1:727-753, 1935.

[7] R. L. Bankert and P. M. Tag. An automated method to estimate tropical cyclone intensity using SSM/I imagery. J. Appl. Met., 41:461-472, 2002.

[8] M. P. Barmin, M. H. Ritzwoller, and A. L. Levshin. A fast and reliable method for surface wave tomography. Pure Appl. Geophys., 158:1351-1375, 2001.

[9] Lt. D. Barr. Personal communication, 2003. 
[10] J. Bayot. First estimate puts storm's economic toll at $\$ 100$ billion. New York Times, Sept. 3, 2005.

[11] P. Bernard. Short review and recent results on microseisms. Geophys. Survey, 5:395-407, 1983.

[12] J. Beven. Tropical cyclone report, hurricane Keith, 28 September - 6 October 2000. Technical report, National Hurricane Center, 2000.

[13] R. T. Beyer. Nonlinear Acoustics, page 101. Acoustical Society of America, Woodbury New York, 1997.

[14] S. P. Bowen, J. C. Richard, J. D. Mancini, V. Fessattidis, and B. Crooker. Microseism and infrasound generation by cyclones. J. Acoust. Soc. Am., 113:25622573, 2003.

[15] H. Bradner, De Jerphanion L. G., and R. Langlois. Ocean microseism measurements with a neutral buoyancy free-floating midwater seismometer. Bull. Seism. Soc. Am., 60:1134-1150, 1970.

[16] L. M. Brekhovskikh. Underwater sound waves generated by surface waves in the ocean. Izv. Atmos. Ocean. Phys., 2(9):582-587, 1966.

[17] W. S. Burdic. Underwater Acoustic System Analysis. Prentice-Hall, Englewood Cliffs, New Jersey, 1984.

[18] W. M. Carey and D. Browning. Low frequency ocean ambient noise: Measurements and theory. In Sea Surface Sound, Dordrecht, 1988. Kluwer Academic Publishers.

[19] W. M. Carey, R. B. Evans, J. A. Davis, and G. Botseas. Deep-ocean vertical noise directionality. IEEE J. Oceanic Eng., 15(4):324-334, 1990. 
[20] D. H. Cato. Ambient sea noise in waters near Australia. J. Acoust. Soc. Am., 60(2):320-328, 1976.

[21] D. H. Cato. Sound generation in the vicinity of the sea surface: source mechanisms and the coupling to the received sound field. J. Acoust. Soc. Am., 89(3):1076-1095, 1991.

[22] D. H. Cato. Theoretical and measured underwater noise from surface wave orbital motion. J. Acoust. Soc. Am., 89(3):1096-1112, 1991.

[23] D. H. Cato and S. Tavener. Spectral differences in sea surface noise in open and enclosed waters. In T. G. Leighton, editor, Natural Physical Processes Associated with Sea Surface Sound, pages 20-27, Southampton, 1997. University of Southampton.

[24] N. R. Chapman and J. W. Cornish. Wind dependence of deep ocean ambient noise at low frequencies. J. Acoust. Soc. Am., 93(2):782-789, 1993.

[25] C. S. Clay and H. Medwin. Acoustics Oceanography: Principles \& Applications. Wiley, New York, 1977.

[26] B. F. Cron and C. H. Sherman. Spatial-correlation functions for various noise models. J. Acoust. Soc. Am., 34:1732-1736, 1962.

[27] J. Darbyshire. Microseisms, in the sea, ideas and observations on progress in the study of the seas. page 701, New York, 1966. Interscience.

[28] L. W. Dean III. Interactions between sound waves. J. Acoust. Soc. Am., 34(8):1039-1044, 1962.

[29] S. Dewan. Louisiana releases details on deaths from hurricane katrina and later flooding. New York Times, Dec. 10, 2005. 
[30] NOAA/AOML/Hurricane Research Division. Hurricane Gert wind analysis 1330 UTC 16 Sept 1999. Technical report, NOAA/AOML/Hurricane Research Division, 1999.

[31] V. F. Dvorak. Tropical cyclone intensity analysis and forecasting from satellite imagery. Mon. Wea. Rev., 103:420-430, 1975.

[32] V. F. Dvorak. Tropical cyclone intensity analysis using satellite data. Tech. Rep. NESDIS 11, NOAA, Washington, DC., 1984.

[33] G. J. Holland (Ed.). Global Guide to Tropical Cyclone Forecasting. World Meteorological Organization, Geneva, 1993.

[34] V. L. Streeter (Ed.). Handbook of Fluid Dynamics. McGraw-Hill, New York, 1961.

[35] D. D. Ellis and D. M. F. Chapman. A simple shallow water propagation model including shear wave effects. J. Acoust. Soc. Am., 78(6):2087-2095, 1985.

[36] K. A. Emanuel. Thermodynamic control of hurricane intensity. Nature, 401:665$669,1999$.

[37] D. K. Smith et al. Hydroacoustic monitoring of seismicity at the slow-spreading Mid-Atlantic ridge. Geophys. Res. Lett., 29(11):13-1-13-4, 2002.

[38] D. L. Evans, D. R. Watts, D. Halpern, and S. Bourassa. Oceanic winds measured from the seafloor. J. Geophys. Res., 89(C3):3457-3461, 1984.

[39] W. M. Ewing, W. S. Jardetzky, and Press F. Elastic Waves in Layered Media, pages 157-189. McGraw-Hill, New York, 1957.

[40] D. M. Farmer and D. D. Lemon. The influence of bubbles on ambient noise in the ocean at high wind speeds. J. Phys. Ocean., 14:1762-1778, 1984. 
[41] Federal Coordinator for Meteorological Services and Supporting Research. National hurricane operations plan. Technical Report FCM-P12-2003, U.S. Dept of Commerce/ Nat. Oceanic and Atmospheric Administration, 2003.

[42] G. Z. Forristall, E. G. Ward, V. J. Cardone, and L. E. Borgmann. The directional spectra and kinematics of surface gravity waves in tropical storm delia. J. Phys. Oceanogr., 8:888-909, 1978.

[43] J. L. Franklin. Tropical cyclone report, hurricane Florence, 10 - 17 September 2000. Technical report, National Hurricane Center, 2000.

[44] J. L. Franklin, L. A. Avila, J. L. Bevin II, M. B. Lawrence, R. J. Pasch, and D. R. Stewart. Eastern North Pacific hurricane season of 2002. Mon. Wea. Rev., 131:2379-2393, 2003.

[45] M. H. Gilmore. Microseisms and ocean storms. Seismological Soc. Bull., 36(2):89-119, 1946.

[46] D. Goodman, Yamamoto T., M. Trevorrow, C. Abbott, A. Turgut, M. Badiey, and K. Ando. Directional spectra observations of seafloor microseisms from an ocean-bottom seismometer array. J. Acoust. Soc. Am., 86:2309-2317, 1989.

[47] B. Gutenberg. Microseisms in north america. Bull. Seism. Soc. Am., 21:1-24, 1931.

[48] E. L. Hamilton. Geoacoustic modeling of the sea floor. J. Acoust. Soc. Am., 68(5):1313-1340, 1980.

[49] E. L. Hamilton. Sound velocity as a function of depth in marine sediments. $J$. Acoust. Soc. Am., 78(4):1348-1355, 1985.

[50] R. M. Hamson. The modeling of ambient noise due to shipping and wind sources in complex environments. Appl. Acoust., 51(3):251-287, 1997. 
[51] K. Hasselmann. A statistical analysis of the generation of microseisms. Rev. Geophy., 1(2):177-210, 1963.

[52] G. J. Holland. An analytic model of the wind and pressure profiles in hurricanes. Mon. Wea. Rev., 108:1212-1218, 1980.

[53] G. J. Holland. The maximum potential intensity of tropical cyclones. J. Atmos. Sci., 54:2519-2541, 1997.

[54] B. Hughes. Estimates of underwater sound (and infrasound) produced by nonlinearly interacting ocean waves. J. Acoust. Soc. Am., 60(5):1032-1039, 1976.

[55] D. Hutt, J. Osler, and D. Ellis. Effect of hurricane michael on the underwater acoustic environment of the Scotian Shelf. In N. G. Pace and F. B. Jensen, editors, Impact of Littoral Environmental Variability on Acoustic Predictions and Sonar Performance, Netherlands, 2002.

[56] Arvello J. I. and Überall H. Adiabatic normal-mode theory of sound propagating including shear waves in a range-dependent ocean floor. J. Acoust. Soc. Am., 88(5):2316-2325, 1990.

[57] F. Ingenito and S. N. Wolf. Site dependence of wind-dominated ambient noise in shallow water. J. Acoust. Soc. Am., 85(1):141-145, 1989.

[58] F. B. Jensen, W. A. Kuperman, M. B. Porter, and H. Schmidt. Computational Ocean Acoustics. Springer-Verlag, New York, 2000.

[59] K. B. Katsaros, P. W. Vachon, W. T. Liu, and P. G. Black. Microwave remote sensing of tropical cyclones from space. J. Oceanography, 58:137-151, 2002.

[60] S. M. Kay. Fundamentals of Statistical Signal Processing, Estimation Theory. Prentice-Hall, Englewood Cliffs, New Jersey, 1993. 
[61] A. C. Kibblewhite. Panel discussion report, wave and turbulence noise. In Sea Surface Sound, Dordrecht, 1988. Kluwer Academic Publishers.

[62] A. C. Kibblewhite and C. Y. Wu. The generation of infrasonic ambient noise in the ocean by nonlinear interactions of ocean surface waves. J. Acoust. Soc. Am., 85(5):1935-1945, 1989.

[63] A. C. Kibblewhite and C. Y. Wu. The theoretical description of wave-wave interactions as a noise source in the ocean. J. Acoust. Soc. Am., 89(5):2241$2252,1991$.

[64] A. C. Kibblewhite and C. Y. Wu. Acoustic source levels associated with the nonlinear interactions of ocean waves. J. Acoust. Soc. Am., 94:3358-3378, 1993.

[65] S. Q. Kidder, M. D. Goldberg, R. M. Zehr, M. DeMaria, J. F. W. Purdom, C. S. Velden, N. C. Grody, and S. J. Kusselson. Satellite analysis of tropical cyclones using the advanced microwave sounding unit (AMSU). Bull. Am. Met. Soc., 81(6):1241-1259, 2000.

[66] B. Kinsman. Wind waves, their generation and propagation on the sea surface, pages 107,336-340. Prentice-Hall, Englewood Cliffs, New Jersey, 1965.

[67] V. O. Knudsen, R. S. Alford, and J. W. Emling. Underwater ambient noise. $J$. Marine Res., 7:410-429, 1948.

[68] G. J. Komen, L. Cavaleri, M. Donelan, K. Hasselmann, S. Hasselmann, and P. A. E. M. Janssen. Dynamics and modelling of ocean waves, page 23. Cambridge University Press, Cambridge, 1994.

[69] S. P. Kumar, T. V. R. Murty, Y. K. Somayajulu, P. V. Chodankar, and C. S. Murty. Reference sound speed profile and related ray acoustics of Bay of Bengal for tomographic studies. Acustica, 80:127-137, 1994. 
[70] W. A. Kuperman and F. Ingenito. Spatial correlation of surface generated noise in a stratified ocean. J. Acoust. Soc. Am., 67(6):1988-1996, 1980.

[71] M. B. Lawrence. Preliminary report, hurricane Gert 11-23 September 1999. Technical report, National Hurricane Center, 2000.

[72] M. B. Lawrence. Tropical cyclone report, hurricane Joyce, 25 september - 2 October 2000. Technical report, National Hurricane Center, 2000.

[73] T. Lay and T. C. Wallace. Modern global seismology, pages 252-254. Academic Press, New York, 1995.

[74] A. W. Lee. The effect of geologic structure upon microseismic disturbance. Roy. Astron. Soc. Mon. Not. Geophys. Suppl., 3:83-105, 1932.

[75] A. W. Lee. On the direction of approach of microseismic waves. Proc. Roy. Soc. London. Ser. A, 886:183, 1935.

[76] W. S. Liggett and M. J. Jacobsen. Covariance of surface-generated noise in a deep ocean. J. Acoust. Soc. Am., 38:303-312, 1965.

[77] S. P. Lloyd. Underwater sound from surface waves according to the LighthillRibner theory. J. Acoust. Soc. Am., 69(2):425-435, 1981.

[78] M. S. Longuet-Higgins. A theory of the origin of microseisms. Phil. Trans. Roy. Soc. Lon. Ser. A, 243(857):1-35, 1950.

[79] N. C. Makris. A foundation for logarithmic measures of fluctuating intensity in pattern recognition. Optics Lett., 20(19):2012-2014, 1995.

[80] N. C. Makris. The effect of saturated transmission scintillation on ocean acoustic intensity measurements. J. Acoust. Soc. Am., 100(2):769-783, 1996. 
[81] N. C. Makris. The statistics of ocean-acoustic ambient noise. In T. Leighton, editor, Sea Surface Sound '97, Dordrecht, 1997. Kluwer Academic Publishers.

[82] N. C. Makris, L. Z. Avelino, and R. Menis. Deterministic reverberation from ocean ridges. J. Acoust. Soc. Am., 97(6):3547-3574, 1995.

[83] N. C. Makris, L. Z. Avelino, and R. Menis. Deterministic reverberation from ocean ridges. J. Acoust. Soc. Am., 97:3547-3574, 1995.

[84] L. Mandel and E. Wolf. Optical coherence and quantum optics, pages 58-59. Cambridge University Press, Cambridge, 1995.

[85] M May. Personal communication, 2004.

[86] M. Miche. Mouvements ondulatoires de la mer en profonder constante ou decroissante. Ann. Ponts Chauss., 114(7):131-164, 1944.

[87] B. J. Mitchell. Monitoring the comprehensive nuclear-test-ban treaty; preface. Pure Appl. Geophys., 158:421-422, 2001.

[88] I.-J. Moon, I. Ginis, T. Hara, H. L. Tolman, C. W. Wright, and E. J. Walsh. Numerical simulation of sea surface directional wave spectra under hurricane wind forcing. J. Phys. Oceanogr., 33:1680-1706, 2003.

[89] P. M. Morse and K. U. Ingard. Theoretical Acoustics, pages 245, 866. Mc-GrawHill, New York, 1968.

[90] J. A. Nystuen and H. D. Selsor. Weather classification using passive acoustic drifters. J. Acoust. Soc. Am., 14:656 666, 1997.

[91] A. Papoulis and S. U. Pillai. Probability, random variables and stochastic processes, page 515. McGraw-Hill, New York, 1965. 
[92] A. Papoulis and S. U. Pillai. Probability, Random Variables and Stochastic Processes, pages 515, 2012-2014. McGraw-Hill, New York, 2002.

[93] R. J. Pasch. Tropical cyclone report, hurricane Debby, 19 - 24 August 2000. Technical report, National Hurricane Center, 2000.

[94] R. J. Pasch. Tropical cyclone report, hurricane Isaac, 21 september - 1 October 2000. Technical report, National Hurricane Center, 2000.

[95] J. S. Perkins, W. A. Kuperman, F. Ingenito, L. T. Fialkowski, and J. Glattetre. Modeling ambient noise in three-dimensional ocean environments. J. Acoust. Soc. Am., 93(2):739-752, 1993.

[96] A. J. Perrone. Deep-ocean ambient-noise spectra in the Northwest Atlantic. $J$. Acoust. Soc. Am., 3(2):762-770, 1969.

[97] A. J. Perrone. Ambient-noise-spectrum levels as a function of water depth. $J$. Acoust. Soc. Am., 48(1):362-368, 1970.

[98] A. D. Pierce. Extension of the method of normal modes to sound propagation in an almost-stratified medium. J. Acoust. Soc. Am., 37(1):19-27, 1965.

[99] A. D. Pierce. Acoustics: An Introduction to Its Physical Principles and Applications. McGraw-Hill, New York, 1981.

[100] A. D. Pierce. Acoustics: An Introduction to Its Physical Principles and Applications. McGraw-Hill, New York, 1981.

[101] W. J. Pierson and L. Moskowitz. A proposed spectral form for fully developed wind seas based on the similarity theory of S. A. Kitaigardski. J. Geophys. Res., 69:5181-5190, 1964. 
[102] C. L. Piggott. Ambient sea noise at low frequencies in shallow water of the Scotian Shelf. J. Acoust. Soc. Am., 36(11):2152-2163, 1964.

[103] S. Pilidou, K. Priestley, E. Debayle, and Ó. Gudmundsson. Rayleigh wave tomography in the north atlantic: high resolution images of the iceland, azores and eifel mantle plumes. Lithos, 79:453-474, 2005.

[104] M. D. Powell, S. H. Houston, and T. A. Reinhold. Hurricane andrew's landfall in south florida. part i: Standardizing measurements for documentation of surface wind fields. Wea. and Fore., 11:304-328, 1996.

[105] F. Press and M. Ewing. A theory of microseisms with geologic applications. Trans. Am. Geophys. Union, 29(2):163-174, 1948.

[106] F. Press and M. Ewing. Propagation of explosive sound in a liquid layer overlying a semi-infinite elastic solid. Geophys., 15:426-446, 1950.

[107] J. E. Ramirez. An experimental investigation of the nature and origin of microseisms at St. Louis, Missouri. Bull. Seis. Soc. Am., 30:35-84, 1940.

[108] P. Ratilal. Remote Sensing of Submerged Objects and Geomorphology in Continental Shelf Waters with Acoustic Waveguide Scattering. PhD dissertation, Massachusetts Institute of Technology, 2002.

[109] P. Ratilal and N. C. Makris. Mean and covariance of the forward field propagated through a stratified ocean waveguide with three-dimensional random inhomogeneities. J. Acoust. Soc. Am., (In review).

[110] M. H. Ritzwoller and A. L. Levshin. Monitoring the comprehensive nucleartest-ban treaty; introduction. Pure Appl. Geophys., 158:1341-1348, 2001. 
[111] P. Roux, K. G. Sabra, W. A. Kuperman, and A. Roux. Ambient noise cross correlation in free space:theoretical approach. J. Acoust. Soc. Am., 117:79-84, 2005.

[112] K. G. Sabra, P. Roux, and W. A. Kuperman. Arrival-time stucture of the timeaveraged ambient noise cross-correlation function in an oceanic waveguide. $J$. Acoust. Soc. Am., 117:164-174, 2005.

[113] K. G. Sabra, P. Roux, and W. A. Kuperman. Emergence rate of the timedomain green's function from the ambient noise cross-correlation function. $J$. Acoust. Soc. Am., 118:3524-3531, 2005.

[114] K. D. K. M. Sarma and B. Mathew. Sound speed structure in the upper layers of equatorial Indian Ocean and central Bay of Bengal during summer monsoon season. J. Acoust. Soc. Ind., 17(3-4):218-221, 1989.

[115] National Weather Service. Hurricane Gert forecast advisory number 17. Technical report, National Weather Service, Miami, 1999.

[116] National Weather Service. Hurricane Gert forecast advisory number 21. Technical report, National Weather Service, Miami, 1999.

[117] N. M. Shapiro, M. Campillo, L. Stehly, and M. H. Ritzwoller. High-resolution surface-wave tomography from ambient seismic noise. Science, 307:1615-1618, 2005.

[118] P. T. Shaw, D. R. Watts, and H. T. Rossby. On the estimation of oceanic wind speed and stress from ambient noise measurements. Deep-Sea Res., 25:1225$1233,1978$.

[119] D. D. Singh. Rayleigh wave group-velocity studies beneath the indian ocean. Bull. Seism. Soc. Am., 95:502-511, 2005. 
[120] S. R. Stewart. Tropical cyclone report, hurricane Michael, 17 - 19 October 2000. Technical report, National Hurricane Center, 2000.

[121] S. R. Stewart. Tropical cyclone report, hurricane Kyle, 20 September - 12 October 2002. Technical report, National Hurricane Center, 2002.

[122] R. Stoneley. The effect of the ocean on rayleigh waves. Geophys. Suppl. Mon. Not. Roy. Astron. Soc., 1(7):349-356, 1926.

[123] C. Subrahmanyam, N. K. Thakur, T. G. Rao, R. Khanna, M. V. Ramana, and V. Subrahmanyam. Tectonics of the Bay of Bengal: New insights from satellite-gravity and ship-borne geophysical data. Earth and Planetary Sci. Lett., 171:237-251, 1999.

[124] F. H. Sutherland, F. L. Vernon, J. A. Orcutt, J. A. Collins, and R. A. Stephen. Results from osnpe; improved teleseismic earthquake detection at the seafloor. Bull. Seism. Soc. Am., 94:1868-1878, 2004.

[125] B. E. Tocholke. Acoustic environment of the Hatteras and Nares abyssal plains, western North Atlantic Ocean, determined from velocities and physical properties of sediment cores. J. Acoust. Soc. Am., 68(5):1376-1390., 1980.

[126] H. L. Toleman. User manual and system documentation of wavewatch-iii version 1.18. Technical Report Tech. Note. 166, cean Modeling Branch, NCEP, National Weather Service, NOAA, U. S. Department of Commerce, 1999.

[127] M. J. Tucker and E. G. Pitt. Waves in Ocean Engineering, pages 33-34. Elsevier, New York, 2001.

[128] R. J. Urick. Principles of Underwater Sound, pages 57, 389. McGraw-Hill, New York, 1983. 
[129] H. L. Van Trees. Optimum array processing. Part IV of detection, estimation and modulation theory, page 95 and 118. Wiley-Interscience, New York, 2002.

[130] C. S. Velden. Observational analyses of North Atlantic tropical cyclones from NOAA polar-orbiting satellite microwave data. J. Appl. Met., 28:59-70, 1989.

[131] C. S. Velden, T. L. Olander, and R. M. Zehr. Developement of an objective scheme to estimate tropical cyclone intensity from digital geostationary satellite imagery. Wea. and Fore., 13:172-186, 1998.

[132] E. J. Walsh, C. W. Wright, D. Vandemark, W. B. Krabill, A. W. Garcia, S. H. Houston, S. T. Murillo, M. D. Powell, P. G. Black, and F. D. Marks Jr. Hurricane directional wave spectrum spatial variation at landfall. J. Phys. Oceanogr., 32:1667-1684, 2002.

[133] J. D. Watkins, R. Ballard, T. A. Beattie, L. Borrone, J. M. Coleman, A. D'Amato, L. Dickerson, P. G. Gaffney II, M. J. Hershman, P. L. Kelly, C. Koch, F. Muller-Karger, E. B. Rasmuson, A. A. Rosenberg, W. D. Ruckelshaus, P. A. Sandifer, and T. Kitsos. Preliminary Report of the U.S. Commission on Ocean Policy. U.S. Commission on Ocean Policy, Washington D.C., 2004.

[134] S. C. Webb. The equilibrium oceanic microseism spectrum. J. Acoust. Soc. Am., 92(4):2141-2158, 1992.

[135] S. C. Webb. Broadband seismology and noise under the ocean. Rev. Geophys., 36:105-142, 1998.

[136] G. W. Wenz. Acoustic ambient noise in the ocean: Spectra and sources. $J$. Acoust. Soc. Am., 34(12):1936-1956, 1962. 
[137] P. J. Westervelt. Scattering of sound by sound. J. Acoust. Soc. Am., 29(2):199$203,1957$.

[138] P. J. Westervelt. Parametric acoustic array. J. Acoust. Soc. Am., 35(4):535-537, 1963.

[139] D. E. Weston. On the losses due to storm bubbles in oceanic sound transmission. J. Acoust. Soc. Am., 86(4):1546-1553, 1989.

[140] J. D. Wilson and N. C. Makris. Full field spatial correlation of range dependent surface generated noise in a stratified ocean with application to hurricane sensing. In 143rd Meeting of the Acoustical Society of America, Pittsburgh, June 2002.

[141] J. D. Wilson and N. C. Makris. Ocean acoustic hurricane classification. J. Acoust. Soc. Am., 119(1), 2006.

[142] C. W. Wright, E. J. Walsh, D. Vandemark, W. B. Krabill, A. W. Garcia, S. H. Houston, M. D. Powell, P. G. Black, and F. D. Marks Jr. Hurricane directional wave spectrum spatial variation in the open ocean. J. Phys. Oceanogr., 31:2472$2488,2001$. 